\title{
Adoption and Impact of Telemental Health on Healthcare Outcomes in the United States
}

Xiaohui Zhao

West Virginia University, xozhao@mix.wvu.edu

Follow this and additional works at: https://researchrepository.wvu.edu/etd

Part of the Health Services Research Commons

\section{Recommended Citation}

Zhao, Xiaohui, "Adoption and Impact of Telemental Health on Healthcare Outcomes in the United States" (2019). Graduate Theses, Dissertations, and Problem Reports. 3884.

https://researchrepository.wvu.edu/etd/3884

This Dissertation is protected by copyright and/or related rights. It has been brought to you by the The Research Repository @ WVU with permission from the rights-holder(s). You are free to use this Dissertation in any way that is permitted by the copyright and related rights legislation that applies to your use. For other uses you must obtain permission from the rights-holder(s) directly, unless additional rights are indicated by a Creative Commons license in the record and/ or on the work itself. This Dissertation has been accepted for inclusion in WVU Graduate Theses, Dissertations, and Problem Reports collection by an authorized administrator of The Research Repository @ WVU.

For more information, please contact researchrepository@mail.wvu.edu. 


\title{
Adoption and Impact of Telemental Health on Healthcare Outcomes in the United States
}

\author{
Xiaohui Zhao \\ Dissertation submitted to the School of Pharmacy \\ at West Virginia University \\ in partial fulfillment of the requirements for the degree of \\ Doctor of Philosophy \\ in \\ Health Services and Outcomes Research \\ Usha Sambamoorthi, $\mathrm{PhD}$, Chair \\ Nilanjana Dwibedi, PhD \\ Traci J. LeMasters, PhD \\ Kim E. Innes, PhD \\ Sandipan Bhattacharjee, $\mathrm{PhD}$ \\ Department of Pharmaceutical Systems and Policy \\ Morgantown, West Virginia \\ 2019
}

Keywords: Telemental health, mental health condition, mental health provider shortage, access to mental health services, healthcare costs

Copyright 2019 Xiaohui Zhao 


\section{ABSTRACT \\ Adoption and Impact of Telemental Health on Healthcare Outcomes \\ in the United States \\ Xiaohui Zhao}

Mental health conditions (MHCs) are the most expensive conditions in the United States (US). The high MHC prevalence coupled with continued undertreatment of MHC has contributed significantly to this high economic burden. Despite policy changes to increase mental health parity and integrate mental and physical healthcare, the shortage of mental health providers remains a significant barrier to mental health $(\mathrm{MH})$ treatment. Telemental health (TMH) has emerged as an alternative care delivery approach to meet the growing demand of $\mathrm{MH}$ care and shortages of $\mathrm{MH}$ providers. Understanding the adoption of TMH among mental health facilities is critical for informing the broad implementation of TMH in the delivery system of mental healthcare. As the use of TMH is determined by not only "supply" but also "demand," it is also important to understand TMH use among adults with MHC. Given that commercial insurance plays an important role in paying for mental health services and telehealth services, it is imperative to examine TMH use and the impact of TMH use on healthcare costs among commercially insured adults with MHC in real-world care settings. Therefore, this dissertation pursued three related aims: 1) to estimate $\mathrm{TMH}$ adoption and examine factors associated with TMH adoption among mental health facilities; 2) to characterize TMH use and identify factors associated with TMH use among commercially insured adults with MHC; and 3) to evaluate how TMH use influences healthcare costs. To comprehensively present TMH state of the art within the current mental health delivery system, the study used diverse data sources. These data sources were: the National Mental Health Services Survey (N-MHSS) for years 2011-2016; a 
geographically diverse claims database for commercially insured adults for years 2010-2017; the latest Area Health Resources File (2016-2017); and national reports of telehealth policies. We found that about one in four mental health facilities in the US adopted TMH in 2016. TMH adoption might be hindered by low facility-level information technology capacity and by state licensure requirements for cross-state telehealth practice. TMH use among adults with MHC was low (16 in 10,000) in this study of a commercially insured population. Current TMH use among adults with MHC was driven mainly by a greater need to access mental health specialists. TMH use was associated with increased MH service use and higher MH-related costs. However, TMH use did not have a significant impact on total third-party healthcare costs. In summary, these findings highlight the need for collaborative efforts by policymakers, payers, and providers to increase the availability of TMH in mental health care delivery, and thereby increase access to mental healthcare services for adults with MHC. Further research is needed to confirm and extend our study findings; specifically, studies are needed to examine how TMH use affects the cost of mental and physical health care separately, and to explore whether TMH use can lead to cost-savings over a longer period among adults with different MHCs. 


\section{Acknowledgement}

First and foremost, I would like to express the deepest appreciation to my major adviser and dissertation chair, Dr. Usha Sambamoorthi, who has guided me, supported me, and encouraged me to pursue my research interests, who has taught me more than I could ever give her credit for here. Without her guidance and persistent help, this dissertation would not have been possible. She is the best mentor and one of the smartest persons that I know. She was and remains my best role model for a researcher. I hope that I could be as lively, enthusiastic, and energetic as her in work and life.

I would also take this opportunity to express my sincere gratitude to my committee members: Dr. Nilanjana Dwibedi, Dr. Traci LeMasters, Dr. Kim Innes, and Dr. Sandipan Bhattacharjee for their time, patience, continuous support, and insightful suggestions. Special thanks to Dr. Usha Sambamoorthi and Dr. Suresh Madhavan in obtaining the great data for my research aims. My sincere thanks also go to all the faculty and graduate students of the PSP department for their consistent support and help. I thank my brilliant batchmates, roommates, and friends: Drishti Shah and Pragya Rai, for the stimulating discussions, for the days and nights we were working together before deadlines, and for all the fun we have had in the past three and a half years. I would also like to thank my colleague and friends, Snow (Xue) Feng and Yifan Zhang, for their consistent support and company.

Last but not least, I am extremely thankful to my parents, my sister, and my husband, Yijun Wang, for their unconditional love, support, and inspiration 


\section{Table of Contents}

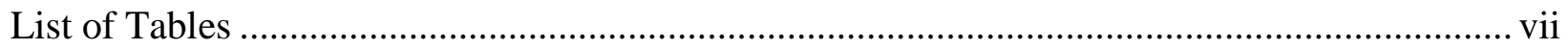

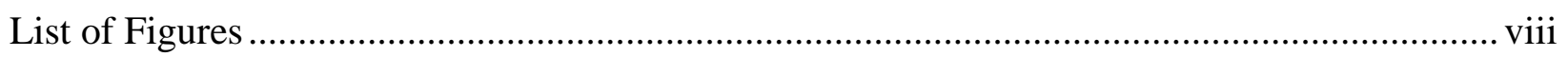

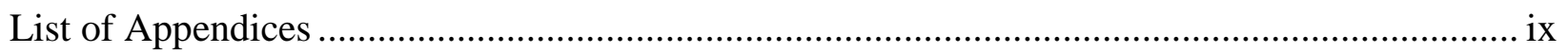

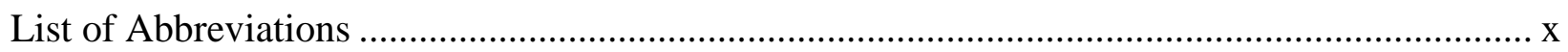

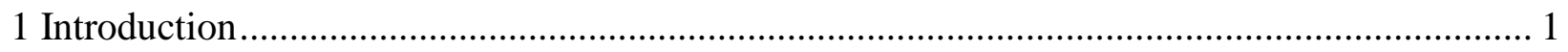

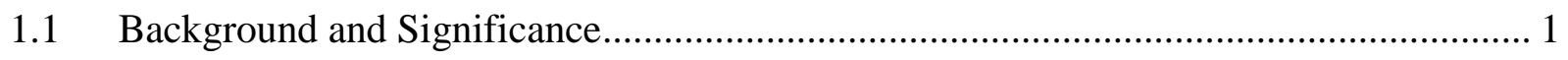

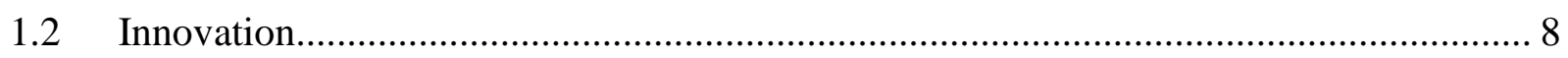

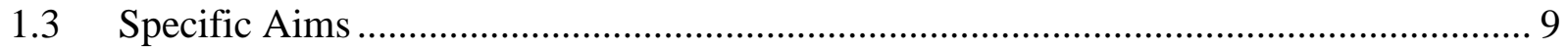

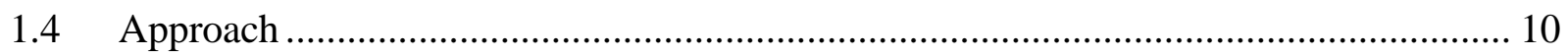

2 Facility and State-level Factors Associated with Telemental Health (TMH) Adoption Among

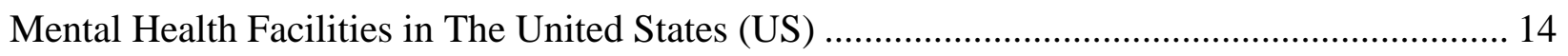

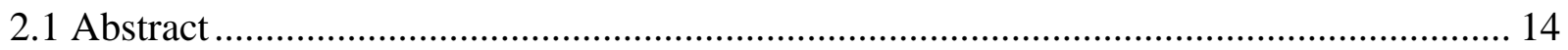

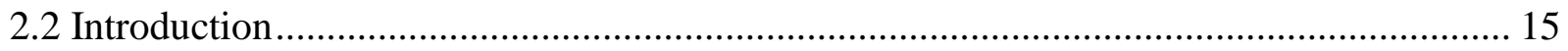

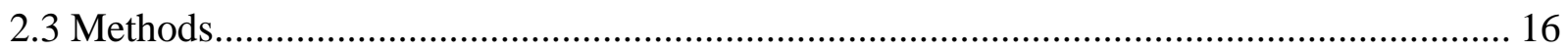

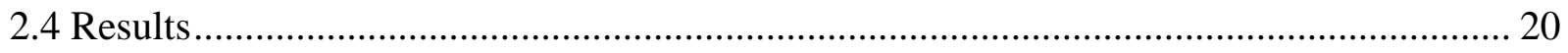

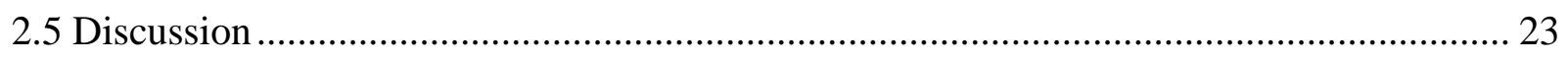

2.6 Declaration of Conflicting Interests and Funding Source................................................. 27

3 Telemental Health Use Among Adults with Mental Health Conditions Enrolled in A

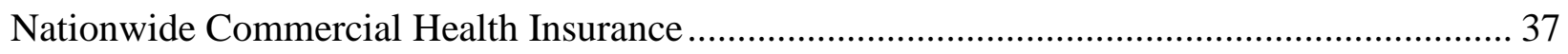

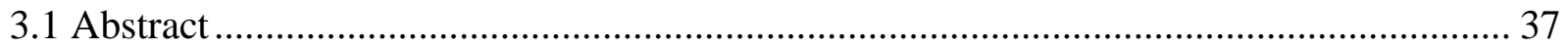

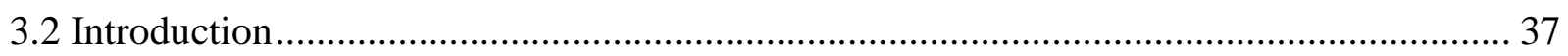

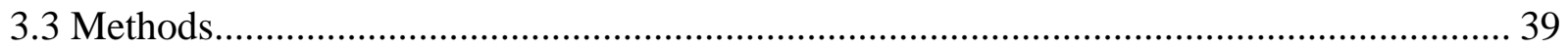

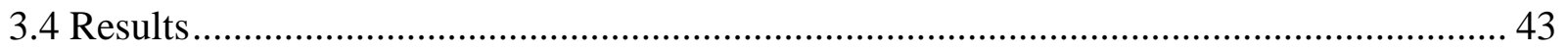




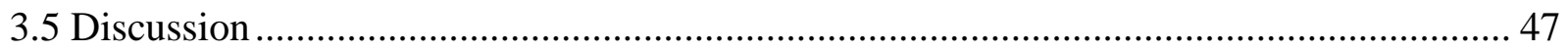

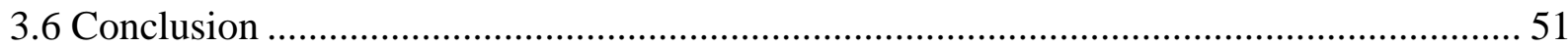

4 The Impact of Telemental Health (TMH) Use on Healthcare Costs Among Commercially

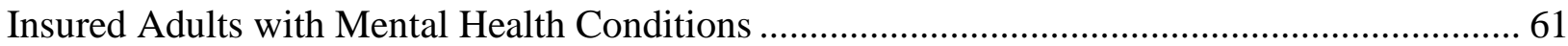

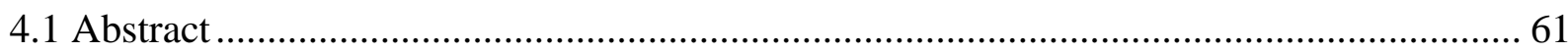

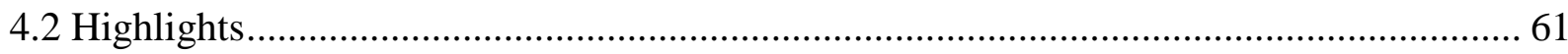

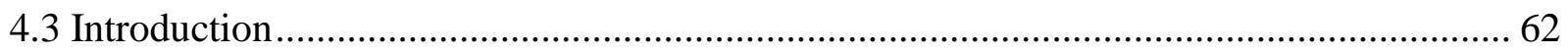

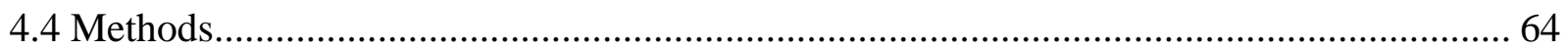

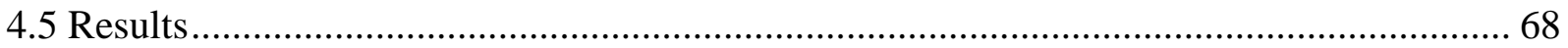

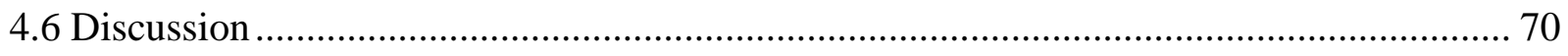

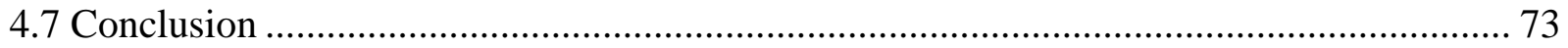

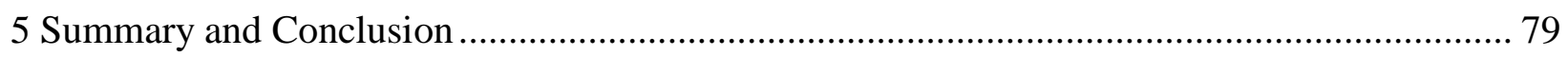

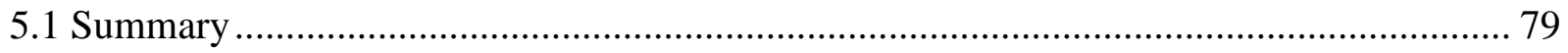

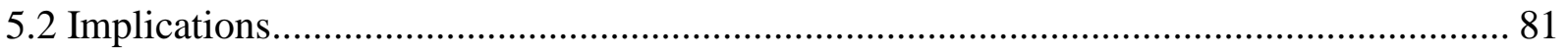

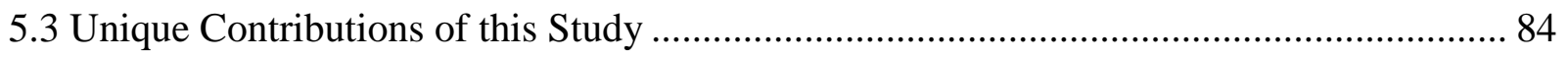

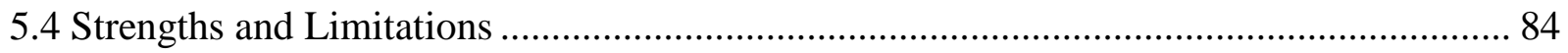

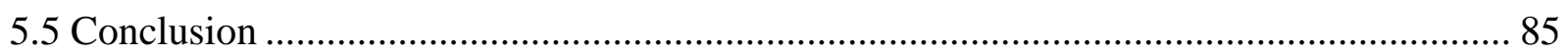

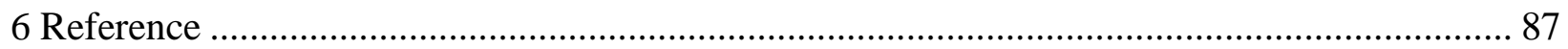

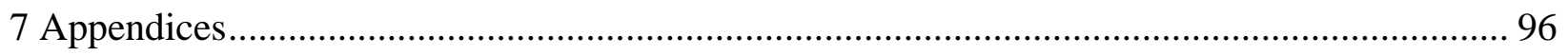




\section{List of Tables}

Table 2.1 Model building process for multi-level multivariable logistic regressions on

Telemental health (TMH) adoption among mental health facilities in the United States .28

Table 2.2 Descriptions of mental health facilities and Telemental health (TMH) adoption among mental health facilities in the United States.

Table 2.3 Adjusted odds ratios (AORs) and 95\% confidence intervals (95\% CI) of facility- and state-level characteristics on Telemental health (TMH) adoption among mental health facilities

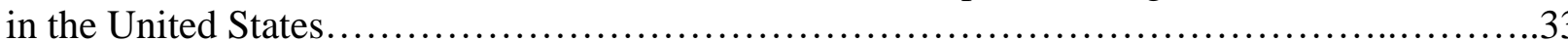

Table 3.1 Description of adults with mental health conditions (MHC) and percentages of Telemental health (TMH) users among adults with MHC.

Table 3.2 Results of multilevel logistic regressions on Telemental health use (yes/no) among adults with mental health conditions (MHCs)

Table 3.3 Results of multilevel logistic regressions on Telemental health use (yes/no) among Medicare Advantage enrollees with mental health conditions (MHC) who lived in underserved states

Table 4.1 Characteristics of adults with mental health conditions (MHCs) who used Telemental health $(\mathrm{TMH})$ and those used in-person mental health $(\mathrm{MH})$ services at baseline and follow-up, Optum Clinformatics ${ }^{\circledR}$ DataMart, 2010-2017............................................. 74

Table 4.2 Unadjusted mean and Standard Deviation (SD) of baseline and follow-up costs (2018 US \$) of adults with mental health conditions (MHC) who used Telemental health (TMH) services and those used only in-person mental health (MH) services, Optum Clinformatics ${ }^{\circledR}$

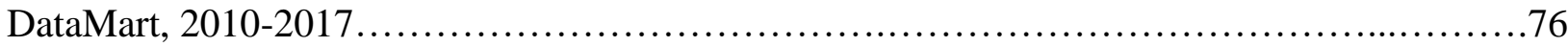

Table 4.3 Parameter estimates of Telemental health use (TMH) from difference-in-differences (DID) analyses for total healthcare costs and $\mathrm{MH}$-related costs among adults with mental health conditions (MHC), Optum Clinformatics ${ }^{\circledR}$ DataMart, 2010-2017..............................77 


\section{List of Figures}

Figure 3.1 Aim 2 Cohort identification process......................................59

Figure 3.2 Distribution of selected mental health $(\mathrm{MH})$ services delivered via Telemental health

$(\mathrm{TMH})$ /in-person.............................................................6

Figure 4.1 Aim 3 Cohort identification process....................................... 78 


\section{List of Appendices}

Appendix 7.1 Procedure codes and modifiers used to identify mental health visits.............95

Appendix 7.2 Chapter 4 supplemental table: Marginal effects estimated from multivariable difference-in-differences (DID) analyses of total healthcare costs and mental health (MH)-related costs among adults with mental health conditions (MHC), Optum Clinformatics ${ }^{\circledR}$ DataMart,

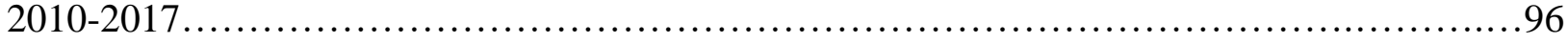




\section{List of Abbreviations}

\begin{tabular}{|c|c|}
\hline AHRF & Area Health Resources File \\
\hline AOR & Adjusted odds ratio \\
\hline ATA & American Telemedicine Association \\
\hline $\mathrm{CCHP}$ & Center for Connected Health Policy \\
\hline CI & Confidence interval \\
\hline $\mathrm{COL}$ & Column \\
\hline CPT & Current Procedural Terminology \\
\hline $\mathrm{DC}$ & District of Columbia \\
\hline DID & Difference-in-differences \\
\hline FFS & Fee-for-service \\
\hline HCPCS & The Healthcare Common Procedure Coding System \\
\hline HIT & Health Information Technology \\
\hline HMO & Health Maintenance Organization \\
\hline ICD-9/10-CM & $\begin{array}{l}\text { International Classification of Diseases, Ninth/Tenth Revision, Clinical } \\
\text { Modification }\end{array}$ \\
\hline IT & Information Technology \\
\hline LR & Log-likelihood \\
\hline MA & Medicare Advantage \\
\hline ME & Marginal effect \\
\hline MH & Mental health \\
\hline MHC & Mental health condition \\
\hline MHPS & Mental health provider shortage \\
\hline MHSA & Mental health and substance abuse \\
\hline N-MHSS & National Mental Health Services Survey \\
\hline NQF & National Quality Forum \\
\hline OOP & Out-of-pocket \\
\hline PCP & Primary care physician \\
\hline PPO & Preferred Provider Organization \\
\hline POS & Point-of-Service \\
\hline QI & Quality Improvement \\
\hline SD & Standard deviation \\
\hline SIG & Significance \\
\hline SUD & Substance use disorder \\
\hline TMH & Telemental health \\
\hline TPM & Two-part Model \\
\hline US & United States \\
\hline VAHC & Veteran Administration Health Center \\
\hline VHA & Veterans Health Administration \\
\hline VPC & Variance partition coefficient \\
\hline
\end{tabular}




\section{CHAPTER 1}

\section{Introduction}

\subsection{Background and Significance}

\section{Epidemiology of Mental Health Conditions}

Mental health is an indispensable part of an individual's overall well-being, interpersonal relationships, and ability to contribute to society. Mental health conditions (MHCs) are a wide range of health conditions characterized by alterations in thinking, mood, and/or behavior that are associated with impaired functioning. ${ }^{1}$ The manifestations of MHCs varies by conditions and individuals, some conditions (e.g., schizophrenia, bipolar disorder, major depressive disorders) can cause serious functional impairments that substantially interferes daily activities. MHC affects 44.7 million, or one in five, adults in the United States (US). ${ }^{2}$ The resulting disease burden of MHCs is among the highest across all diseases, accounting for about one-third (32.4\%) of years lived with disability globally. ${ }^{3,4}$ Furthermore, individuals with MHC die 8 - 32 years earlier than those without any MHC.,6

\section{The Burden of Untreated MHCs}

Although MHCs can be extraordinary disabling, they can be managed with proper treatments. ${ }^{7}$ However, a substantial proportion of adults with MHCs remain untreated. Data from the 2016 National Survey on Drug Use and Health indicated that $56.9 \%$ of adults with MHC did not receive any treatments in the previous year. ${ }^{2}$ Untreated MHCs can have a series of consequences, including worsening the condition and making treatment harder, leading to the development of chronic physical conditions (e.g., cardiovascular diseases), impairing quality of life, and increasing suicidal ideation. ${ }^{8}$ According to the National Alliance on Mental Illness, untreated mental illnesses cost about $\$ 100$ billion a year due to productivity loss. ${ }^{9}$ 


\section{Barriers to Mental Health Treatments}

Despite policy changes to increase mental health parity and integrate mental and physical healthcare, ${ }^{10,11}$ the shortage of mental health providers remains a significant barrier to mental health $(\mathrm{MH})$ treatments. ${ }^{12,13}$ As of 2017, there were 5,042 designated Mental Health Care Health Professional Shortage Areas in the US. ${ }^{14}$ It is reported that over three-quarters (77\%) of US counties had a severe shortage of mental health providers. ${ }^{15}$ Insufficient mental health providers can lead to a long waiting time for patients seeking mental health treatments. A recent study assessing the access to and attitudes towards mental health services reported that over one-third (38\%) of adults with MHCs had to wait longer than one week for mental health treatments. ${ }^{16}$ Timely access to mental health services is critical to successful treatment, ${ }^{17}$ and timelines of care is a key indicator of quality healthcare. ${ }^{18}$ Therefore, addressing the shortage of mental health providers is a critical step in improving mental healthcare in the US.

Other commonly reported barriers included geographical isolation and stigma associated with visiting mental health providers. ${ }^{16,19}$ A study reported that $46 \%$ of adults with MHC had to drive more than an hour to seek mental health services. ${ }^{16}$ The study also revealed that nearly onethird (31\%) of adults with MHC reported worries about being judged when they have sought mental health services. ${ }^{16}$

\section{Telehealth and Access to Care}

Telehealth is rising as a promising solution to provider shortage and limited access to care due to geographic barriers. ${ }^{20}$ Telehealth, often referred to as telemedicine, has many definitions. Commonly it is defined as "the use of medical information exchanged from one site to another via electronic communications to improve a patient's clinical health status." ${ }^{21}$ Two forms of telehealth, namely live interactive video (synchronous telehealth) and store-and-forward 
electronic information exchange (asynchronous telehealth), are currently paid by insurers and therefore often used in clinical care for consultation, diagnosis, and treatments. ${ }^{22-25}$ Results from a systematic review assessing 106 telehealth interventions across various disease areas concluded that telehealth was most effective for managing care conditions that do not primarily rely on physical examinations. Thus, telehealth is considered ideal for managing MHCs. ${ }^{26}$

\section{Telemental health (TMH) and Access to Mental Healthcare}

The application of telehealth in mental healthcare is referred as telemental health (TMH), which includes telepsychology, telepsychiatry, and telebehavioral health. ${ }^{27}$ Current TMH applications include mental health assessment, treatment, education, monitoring, and collaboration among providers. ${ }^{28}$ It can be offered by a wide range of providers: primary care physicians, psychiatrists, psychologists, nurse practitioners, social workers, and counselors. ${ }^{28}$ TMH has been found effective in removing geographic barriers to mental health treatments by maximizing the reach of the existing mental health professionals. Furthermore, TMH can expand the capacity of primary care settings in delivering mental health care by bridging the communications among mental health professionals, primary care physicians, and patients. A recent systematic review of 134 studies on TMH concluded that TMH interventions were effective in improving access to mental health care, especially for those living in isolated areas. ${ }^{29}$ Given that many adults with MHC do not seek treatment due to the stigma associated with visiting mental health providers, evidence also suggests that TMH may also increase access by helping adults with MHCs feel less stigmatized. ${ }^{30-32}$

\section{Adoption of Telehealth and TMH among Healthcare Providers}

The significant advances in information and communication technologies have accelerated the adoption and use of telehealth in general among healthcare providers and 
facilities. Data from the American Hospital Association annual survey of acute care hospitals showed that $42 \%$ of US hospitals have some forms of telehealth services by $2012 .{ }^{39}$ The adoption of telehealth was affected by the type of facility, rurality, population density, and state policies. Teaching hospitals, those equipped with advanced medical technologies, non-profit institutions, and those belong to a larger hospital system were more likely to have telehealth capacities. ${ }^{39}$ Facilities in rural areas have higher adoption rates than those in urban areas. ${ }^{39}$ The pass of telemedicine parity laws that require private payers to reimburse for telehealth services to the same extent as in-person services were associated with higher odds of adoption, whereas state policies that require out-of-state providers to have special licenses when delivering telehealth services to Medicaid patients reduced the odds of adoption. ${ }^{39}$

The first step in the wide-spread implementation of TMH in delivering mental health treatments is the adoption of TMH among mental health facilities. However, little is known about TMH adoption among mental health facilities. Such knowledge is warranted to inform policy-making and healthcare resources allocation. This leads to the rationale for Aim 1, which estimated the adoption rate of TMH and examined state- and facility-level factors associated with TMH adoption among mental health facilities.

\section{TMH Use among Adults with MHCs}

Recognizing the potential for TMH to remove access-related barriers to treatment, federal, state, and local governments have made substantial efforts to increase TMH services in recent years. ${ }^{40}$ However, only a few studies have examined TMH use among adults with MHCs in real-world settings using recent data. One study analyzing 2008 and 2009 Medicaid claims reported very low $(<0.1 \%) \mathrm{TMH}$ use and high state-level variations due to differences in coverage and reimbursement policies for TMH across states. ${ }^{41}$ Notable variations in TMH use 
were also reported in a study analyzing 2004-2014 claims data of Medicare beneficiaries with MHCs, who had the same TMH coverage and reimbursement policies. ${ }^{42}$ While these studies illustrated the role of reimbursement policies in TMH use, they also highlighted the wide variation in TMH use among states, despite having the same reimbursement and coverage for TMH services. This finding suggests that patient characteristics and state-level factors may also contribute to TMH use. Previous studies have reported that TMH use was significantly associated with younger age, having any disability, and living in a poor community. ${ }^{43,44}$ Health plan characteristics (e.g., out-of-pocket expenditures ${ }^{45}$ ), factors that may increase the need for TMH/MH services (e.g., previous treatment seeking, ${ }^{46}$ polypharmacy ${ }^{47}$ ), and environmental capacities that allow TMH use were not explored.

\section{Adoption of Telehealth and TMH among Healthcare Payers}

Like any other healthcare services, telehealth adoption by healthcare payers is essential to the overall deployment of telehealth. The overall reimbursement environment for telehealth services is improving over time in both public and private sectors, with large variation by payers. ${ }^{33}$ The Veterans Health Administration (VHA) is the largest payer of telehealth services, including TMH, in the US. The VHA telehealth programs encompass 44 clinical specialties in various clinical settings (e.g., emergency care, intense care unit). ${ }^{34}$ Unlike the VHA, the Medicare fee-for-service (FFS) programs limit reimbursement and coverage for telehealth by types of healthcare professionals, modalities of telehealth services, geographic locations where patients receive care (i.e., originating site) ${ }^{35,36}$ For example, Medicare FFS programs cover only cover synchronous TMH services for beneficiaries living in rural areas or areas with shortages of providers. ${ }^{35,36}$ Medicare Advantage (MA) plans may cover telehealth services and/or areas 
beyond the FFS programs' coverage after the approval of the Centers for Medicare and Medicaid Services. ${ }^{35,36}$

Telehealth reimbursement policies vary widely in Medicaid programs and private insurances. As for 2017, all 50 state Medicaid programs have some type of coverage for TMH. ${ }^{37}$ Private insurers have embraced telehealth better than public insurers,${ }^{38}$ with all top-ten health insurance companies have covered TMH in their plans. There is evidence showing that private payers are more likely to be billed for telehealth services than public payers. ${ }^{38}$

While private insurance provides an important source of health coverage for adults with MHCs ${ }^{48}$ knowledge about TMH use among commercially insured adults with MHC is limited. Although TMH has been covered for almost all MH services from various providers (e.g., psychiatrists, social worker, physicians), ${ }^{49}$ how TMH is used in various MH services and who is delivering TMH in real-world practice remained unknown. Such knowledge is vital to inform health insurers and healthcare policymakers how to better incorporate TMH in delivering mental health services into real-world healthcare settings. These knowledge gaps lead to the rationale for Aim 2, which estimated TMH use, explored the role of TMH use in delivering MH services, and examined the associations of patient- and state-level factors to TMH use among adults with MHCs using linked claims data from a large U.S. commercial plan.

\section{The Comparative Efficacy of TMH versus In-person Services}

Results of randomized clinical trials and systematic reviews have demonstrated that TMH in various modalities (e.g., internet, phone, video-conferencing) is comparable or even superior to in-person care in assessing and treating adults with MHCs across different diagnostic and demographic groups. ${ }^{50-54}$ The majority of TMH studies have focused on anxiety and mood disorders, probably due to their high prevalence and their amenability to remote treatments. ${ }^{54}$ 
Specifically, there is consistent evidence revealing that TMH is associated with higher treatment adherence and improved depressive symptoms as compared to in-person care. ${ }^{55-57}$ Studies evaluating TMH in assessing and treating serious MHCs such as schizophrenia and bipolar disorders are also rising. ${ }^{58,59}$ Preliminary evidence suggested that TMH appeared to improve outcomes of serious MHCs. ${ }^{58,59}$

\section{The Impact of TMH use on Healthcare Outcomes in Real-world Care Settings}

Although the comparative efficacy of TMH in delivering MH care as compared to inperson care is well-documented in clinical trials, real-world evidence on healthcare outcomes associated with TMH use among adults with MHCs was lacking. Existing evidence primarily comes from studies using data from the VHA. A study examining outcomes of 98,609 veterans receiving TMH services reported a $25 \%$ reduction in psychiatric admissions and hospital stays after initiating TMH over four years. ${ }^{60}$ However, the study did not have individuals receiving only in-person MH services as controls. ${ }^{60}$ Studies comparing outcomes among MHC patients receiving TMH and in-person MH services are warranted to establish the value of TMH services.

Given the limited healthcare resources, healthcare costs have always been an essential measurement of healthcare outcomes in the US. In response to the rising healthcare costs, the Institute of Health Improvement developed the "Triple Aim" to define high-value care as those can improve population health and individual care experience with reduced per-capita costs. ${ }^{61}$ To be aligned with the "Triple Aim," the National Quality Forum (NQF) and the American Telemedicine Association (ATA) used healthcare costs as one of the key measurements in their newly developed telehealth value framework. ${ }^{62}$

Existing studies have primarily focused on indirect medical costs such as time and expenses related to travel. TMH has been found effective in reducing the costs of traveling 
among patients as well as providers. ${ }^{63-66}$ No study has compared direct healthcare costs between MHC patients who used TMH services and those used only in-person MH services. Such knowledge can provide valuable insights to healthcare payers regarding the expansion of TMH coverage.$^{67}$ This leads to the rationale of aim 3 , which examined the association of TMH use with direct healthcare cost among adults with MHC by comparing the total and MH-related costs among adults with MHC who used TMH services and those used in-person MH service only from a commercial insurance's perspective.

\subsection{Innovation}

a) Comprehensive analysis of the factors associated with TMH adoption among mental health facilities: This is the first study to estimate the TMH adoption rate and examine state- and patient-level factors associated with TMH adoption among mental health facilities in the US with a linked population-based data.

b) Examination of the association between state-level TMH capacity and TMH use among commercially insured adults with MHCs in the US: This is the first study to examine the association between the state-level adoption of TMH among mental health facilities and TMH use among adults with MHCs who were enrolled in a geographically diverse commercial health insurance in the US.

c) Description of the role of TMH in delivering mental health services: the first study to characterize the role of TMH in delivering various mental health services among adults MHCs enrolled in a large commercial health insurance in the US.

d) Application of econometric techniques to evaluate the impact of TMH use on total third-party healthcare costs and MH-related costs: This is the first study to evaluate 
how TMH use influences total healthcare costs MH-related costs from a third-party payer's perspective.

e) Use of a geographically diverse data of commercially insured adults with MHCs: Data from the Optum Clinformatics ${ }^{\circledR}$ DataMart allows the examination of TMH use among commercially insured adults with MHCs across all 50 states and the District of Columbia.

f) Use of standard prices of healthcare services and prescription drugs to measure third-party healthcare costs: The standard prices provided by Optum Clinformatics ${ }^{\circledR}$ DataMart estimated the allowed payments from insurers with standard pricing algorithms that account for types of services, the quantity of services, and relevant resources cost involved in providing the services. In this way, the healthcare costs of commercially insured adults with MHCs from different geographic areas and those enrolled in different insurance plans can be compared in a consistent manner.

\subsection{Specific Aims}

AIM 1: Estimate TMH adoption rates and examine state- and facility-level characteristics associated with TMH adoption among mental health facilities.

Hypothesis: TMH adoption will be more likely in facilities with non-profit ownership, higher IT capability, more elderly patients, and those located in states with friendly telehealth policies and higher levels of provider shortage.

\section{AIM 2: Characterize TMH use and identify state- and patient-level factors associated with}

\section{TMH use among commercially insured adults (age $\geq 18$ years) with MHCs.}

Hypothesis: $\mathrm{TMH}$ will be more likely to be used by MHC patients who are younger, enrolled in managed care plans, and those with severe MHCs; Patients residing in states 
that have a higher percentage of rural counties or higher provider shortage will be more likely to use TMH as compared to those residing in states that are less rural and with a lower provider shortage.

\section{AIM3: Evaluate the impact of TMH use on total third-party healthcare costs and MH- related costs among commercially insured adults (age $\geq 18$ years) with MHCs.}

Hypothesis: TMH use will increase MH-related healthcare costs but will not influence total third-party healthcare costs.

\subsection{Approach}

\section{Conceptual Framework}

Aim 1: The conceptual framework for Aim 1 was built on common constructs and factors that have been examined in previous studies on telehealth, electronic medical record (HER), and other health information technologies (HITs). ${ }^{68-70}$ The model posits that the adoption of TMH by a healthcare facility is a result of multilevel factors, including environmental resources, policy-, market-, and facility-level characteristics. The environmental resource most pertinent to TMH use is the local broadband access (25 Mbps/3 Mbps service). ${ }^{71}$ Policy characteristics include 1 ) state-level coverage and reimbursement policies for $\mathrm{TH}$; 2) licensure policies that require providers to have licenses for cross-state $\mathrm{TH}$ practice, and 3) state consent policies that require some informed consent by patients before receiving TH services. Market characteristics consist of factors that influence the demand for TMH. Such factors include the level of rurality and the shortage of health care providers (i.e., primary care and mental health). Facility characteristics that may affect TMH adoption can be grouped into five domains: 1) facility type (e.g., care setting, ownership, affiliation, and volume of patients); 2) comprehensiveness of mental health treatment (e.g., treatment focus, the number of mental health treatment approaches, services, and 
special programs, the availability of non-English services); 3) quality improvement practice 4) Information Technology capacity; and 5) payer-mix (e.g., Medicare, Medicaid, Veteran Health Administration) and case-mix, which includes the demographic composition (e.g., age, sex, race/ethnicity) and percentages of high-need patients (e.g., veterans, patients with co-occurring mental health and substance abuse).

Aim 2: The conceptual framework of Aim 2 was adapted from the determinants of health model proposed by Wilkinson and Marmot, ${ }^{72}$ and the Telehealth Research and Policy Framework proposed by a multidisciplinary group of experts and literature review. ${ }^{73}$ The model suggests that factors that affect TMH use can be grouped into four domains, including 1) biological factors (e.g. age, sex); 2) health insurance characteristics (e.g., Medicare Advantage Enrollment, type of health plan, out-of-pocket expenditures); 3) need factors (e.g., severity of MHC, any mental health specialist visit, physical chronic conditions, polypharmacy, substance use disorders, obesity); as well as 4) environmental factors (e.g. poverty, rurality, healthcare provider shortage, TMH capacity).

Aim 3: The conceptual framework of Aim 3 was based on the adapted Andersen's Behavioral Model of Health Services Use ${ }^{74}$ which posits that healthcare utilization of an individual is a function of multiple factors. These factors can be grouped into four domains, including 1) predisposing factors that represent the individual characteristics predictive of using healthcare services (e.g., age, sex); 2) enabling factors that may enable access to healthcare services (e.g., TMH use, type of health insurance, type of insurance plan); 3) need factors that define an individual's health status (e.g., severe MHC diagnosis, mental health specialist visits, the number of physical chronic conditions, the presence of polypharmacy, obesity, and any substance use disorders); and 4) environmental factors that may influence an individual's 
healthcare utilization (e.g., \% counties under poverty, \% rural counties, \% counties with $\mathrm{MH}$ provider shortage, and \% MH facilities with TMH adoption).

\section{Data Sources}

To accomplish the study objectives, we used data from multiple sources. To estimate the TMH adoption and examine state- and facility-level factors associated with TMH adoption among mental health facilities (Aim 1), we used linked data from five sources: 1) 2016 National Mental Health Services Survey (N-MHSS); 2) the 2016-2017 Area Health Resources File (AHRF); 3) the 2016 Broadband Progress Report from the Federal Communications Commission; ${ }^{75}$ 4) the State Telemedicine Gaps Analysis: Coverage \& Reimbursement from the ATA $;{ }^{76}$ and 5) the State Telehealth Laws and Reimbursement Policies from the Center for Connected Health Policy (CCHP). ${ }^{77}$ The N-MHSS is an annual survey conducted by the Substance Abuse and Mental Health Services Administration of all known public and private mental health treatment facilities throughout all 50 states, the District of Columbia, and other US jurisdictions. It is the only source of national and state-level data on the mental health services delivery system for publicly- and privately-operated specialty mental health care facilities. ${ }^{78} \mathrm{~N}-$ MHSS collects a comprehensive set of information on facility type, primary treatment focus, mental treatment characteristics, management characteristics such as computerized functionality, payer mix, licensing, certification, or accreditation, as well as demographics of patients. ${ }^{78}$ The AHRF include data on healthcare providers, population characteristics and environment at the county, state and national levels from over 50 data sources.

To characterize TMH use, identify state- and patient-level factors associated with TMH use (Aim 2), and evaluate the impact of TMH use on healthcare costs (Aim 3), we used linked data from several sources: 1) de-identified 10\% sample of the Optum Clinformatics ${ }^{\circledR}$ DataMart 
(Optum Clinformatics ${ }^{\circledR}$, Eden Prairie, MN, USA) from $1^{\text {st }}$ January 2010 through $30^{\text {th }}$ June 2017; 2) 2016-2017 AHRF; and 3) the 2010 - 2016 N-MHSS. Optum Clinformatics ${ }^{\circledR}$ provides deidentified paid medical and prescription claims for individuals enrolled in the commercial insurance plans and Medicare Advantage plan of a large U.S. health insurance company. This databases also encompass demographic characteristics (i.e., year of birth, gender, residential state) and plan type (e.g., health maintenance organization, preferred provider organization, point of service, fee-for-service) that are routinely collected during health insurance enrollment. ${ }^{79}$ 


\section{CHAPTER 2}

\section{Facility and State-level Factors Associated with Telemental Health (TMH) Adoption Among Mental Health Facilities in The United States (US)}

\subsection{Abstract}

Introduction: Telemental health $(\mathrm{TMH})$ is a promising approach to increase access to mental healthcare. This study examined the TMH adoption rates and associations with facility and statelevel factors among US mental health (MH) facilities. Methods: This retrospective, crosssectional study used linked data for 2016 from the National Mental Health Services Survey $(\mathrm{N}=$ 11,833), Area Health Resources File, and national reports for broadband access and telehealth policies. The associations of facility and state-level characteristics with TMH adoption were examined with multi-level logistic regressions. Results: Overall, 25.9\% had adopted TMH. Having veteran affiliation [Adjusted Odds Ratio $(A O R)=18.53,95 \%$ Confidence Interval (95\%CI): 10.66-32.21] and high Information Technology (IT) capacities [AOR (95\%CI): 2.89(2.10-3.98)] were the strongest correlates of TMH adoption. Other facility characteristics indicating higher TMH adoption were: having public ownership, high patient volumes, comprehensive MH treatments or Quality Improvement practices, private or non-Medicaid public payers, and treating elderly patients (AORs: $1.16-2.41$ ). The adoption was less likely among facilities treating more patients with substance abuse disorders or African Americans. TMH adoption varied substantially across states, with adoption more likely in states issuing special telehealth licenses and those with more rural counties. Discussion: One in four $\mathrm{MH}$ facilities adopted TMH in 2016. TMH adoption varied by multiple facility- and state-level factors. Our findings suggest that: legal/regulatory burden and lower facility IT capacity may 
discourage TMH adoption; significant racial disparities exist in $\mathrm{TMH}$ access, and there is a need to increase TMH use for substance use disorders.

\subsection{Introduction}

Mental health conditions (MHCs) affect 44.7 million adults in the $\mathrm{US}^{2}{ }^{2}$ one in five adults experience an MHC in a given year. ${ }^{2}$ Although MHCs can be highly disabling, they can be managed with proper treatments. ${ }^{7}$ Despite policy changes to increase mental health parity and integrate mental and physical healthcare, ${ }^{10,11} 50 \%$ of adults with MHCs remain untreated. ${ }^{2}$ Untreated MHCs can impose a heavy burden on individuals, their families, payers, and society. ${ }^{8,80}$ The shortage of mental health providers remains a significant barrier to mental health $(\mathrm{MH})$ treatments. ${ }^{12,13}$ As of 2017, there were 5,042 designated Mental Health Care Health Professional Shortage Areas in the US. ${ }^{14}$

Tele-mental health (TMH), the delivery of $\mathrm{MH}$ services through remote technologies (e.g., video-based conferencing), is emerging as a promising solution to the shortage of providers. ${ }^{27}$ Existing studies have demonstrated the efficacy of TMH in removing geographic barriers to $\mathrm{MH}$ treatments ${ }^{60,81}$ and facilitating the collaboration between primary care providers and mental health providers. ${ }^{82}$ Given that many patients do not seek $\mathrm{MH}$ treatment due to the stigma associated with in-person visits to mental health facilities, TMH may also increase treatment seeking by "bringing" mental health providers to primary care settings. ${ }^{30-32}$ Furthermore, a recent review of eight systematic reviews of TMH interventions indicated that TMH was comparable to in-person care in assessing and treating various mental health conditions. ${ }^{83}$ As TMH has great potential for improving mental healthcare delivery, it is important to understand how TMH is implemented in real-world healthcare settings. 
The first step in the wide-spread implementation of TMH is the adoption of TMH in mental health facilities. Adoption of TMH may be determined by multiple factors. However, rigorous studies of TMH adoption rates are lacking. Likewise, although several studies have investigated factors associated with the adoption of Electronic Medical Record (EMR) system and other Health Information Technologies (HITs), ${ }^{39,68-70}$ little is known about the factors associated with TMH adoption. Such knowledge is needed to inform healthcare policy and allocation of healthcare resources as well as to improve healthcare outcomes of patients with mental health conditions through TMH-affiliated collaborative care. Therefore, this study aimed to (1) estimate the TMH adoption rate and (2) examine facility and state-level factors associated with TMH adoption among mental health facilities in the US using linked population-based data.

\subsection{Methods}

\section{Study design and data sources}

This retrospective cross-sectional study used linked data from five sources: 1) 2016 National Mental Health Services Survey (N-MHSS); 2) the 2016-2017 Area Health Resources File (AHRF); 3) the 2016 Broadband Progress Report from the Federal Communications Commission; ${ }^{75}$ 4) the State Telemedicine Gaps Analysis: Coverage \& Reimbursement from the American Telemedicine Association (ATA); ${ }^{76}$ and 5) the State Telehealth Laws and Reimbursement Policies from the Center for Connected Health Policy (CCHP). ${ }^{77}$ The N-MHSS is an annual survey conducted by the Substance Abuse and Mental Health Services Administration (SAMHSA) of all known public and private mental health treatment facilities throughout all 50 states, the District of Columbia, and other US jurisdictions. It is the only source of national and state-level data on the mental health services delivery system for publicly and privately operated mental health care specialty facilities. ${ }^{78} \mathrm{~N}$-MHSS collects a comprehensive 
set of information on facility type, primary treatment focus, mental treatment characteristics, management characteristics such as computerized functionality, payer mix, licensing, certification, or accreditation, as well as demographics of patients. ${ }^{78}$ The survey universe was identified from the database produced after fielding the 2010 and $2014 \mathrm{~N}-\mathrm{MHSS} .{ }^{78}$ The $2016 \mathrm{~N}$ MHSS was conducted from March 2016 through January 2017 among 13,983 facilities across the nation with a response rate of $91 \%$. A total of 12,172 facilities were eligible for reporting in the final dataset. ${ }^{78}$

The Area Health Resources File (AHRF) for 2016 was used to identify state-level population characteristics and healthcare resources. The AHRF data provide county, state and national-level data on health care professions, health facilities, population characteristics, and the general environment from over 50 valid data sources, including the American Medical Association, the American Community Survey, and the U.S. Census Bureau. ${ }^{84}$

\section{Study population}

The study population included 11,883 mental health facilities located in the 50 states and the District of Columbia that responded to the query about TMH use and patient characteristics in the 2016 N-MHSS. Facilities in American Samoa, Guam, Puerto Rico, and the Virgin Islands were excluded due to insufficient information on telehealth policies $(\mathrm{N}=99)$.

\section{Measures}

\section{Dependent Variable: TMH adoption (Yes/No)}

The dependent variable was TMH adoption of mental health facilities. A binary variable (yes/no) was created based on the responses of the N-MHSS question "which of these mental health treatment approaches are offered at this facility, at this location?" from the facility director. The survey provided a checklist of items and facilities that checked the item of 
"telemedicine therapy" were identified as facilities with TMH adoption, whereas those that did not check this item were identified as facilities without TMH adoption.

\section{Explanatory Variables:}

The explanatory variables were selected based on the common constructs and factors that have been examined in previous studies on telehealth, EMR, and other HITs. ${ }^{39,68-70}$ Consistent with the findings of these studies, we hypothesized that the adoption of TMH by a mental health facility may be associated with multiple state-and facility-level factors, including environmental resources, policy, market, and facility-level characteristics.

Facility-level characteristics were measured in five domains: 1) facility type that included care setting, ownership, affiliation, and volume of patients; 2) comprehensiveness of mental health treatment, including treatment focus, the number of mental health treatment approaches, services, and special programs as well as the availability of non-English services; 3) quality improvement (QI) practice; 4) Information Technology (IT) capacity; and 5) payer mix [Medicare/Medicaid/Veteran Health Administration (VHA)] and case-mix, which included demographic composition (e.g., age, sex, race/ethnicity) and percentages of high-need patients [e.g., \% veteran patients and \% patients with co-occurring mental health and substance abuse (MHSA)]. The IT capacity was defined as the level of technology involvement in 14 routine facility activities. The level of technology involvement was measured by the mean score of all activities rated on a 3-point scale, where "1" denotes no technology involvement (i.e., rely on paper), "2" indicates partial involvement (i.e., both electronic and paper), and "3" represents fully involvement (i.e., only electronic).

State-level characteristics included environmental resources, telehealth policy, and market characteristics. Environmental resources were measured as the percentage of counties 
with broadband access (25Mbps/3 Mbps service) based on the 2016 Broadband Progress Report. ${ }^{75}$ The telehealth policy environment was measured by three factors: 1) reimbursement and coverage of telehealth; 2) licensure policies that require providers to have special licenses for cross-state telehealth practice, and 3) consent policies that require informed consent by patients before receiving any telehealth services. We used the ATA grading $(\mathrm{A} / \mathrm{B} / \mathrm{C} / \mathrm{F})$ to represent the overall reimbursement and coverage environment of each state. ${ }^{76}$ The summary grades were developed based on 13 indicators that included telehealth parity, Medicaid policies, and innovative payment models. ${ }^{76}$ The licensure and consent policy for each state were obtained from CCHP's State Telehealth Laws and Reimbursement Policies Report. ${ }^{77}$ We categorized the licensure policy of a state into five groups: 1) requiring individual state license, 2) adopting the Federation of State Medical Boards (FSMB)'s Interstate Medical Licensure Compact policy that allows for an expedited application for licenses in participating states; 3) issuing special telehealth license, 4) having exemptions for acceptable practice or states, and 5) not requiring licenses or policy not defined. The consent requirement of a state was dichotomized as "yes (i.e., consent needed)" and "no (i.e., consent not needed)." Market characteristics were derived from the 2016-2017 AHRF and included factors that influence the demand for TMH: the level of rurality represented by the percentage of rural counties (2013 rural-urban continuum code of 8 and 9) and provider shortages for primary care providers (PCP) and mental health providers (MHP). ${ }^{84}$ We categorized provider shortage into three groups based on percentages counties that were designated as shortage areas in the state: 1 ) high (states ranked on $4^{\text {th }}$ quartile for both PCPs and MHPs were considered having high level of shortage); 2) moderate (states ranked on $2^{\text {nd }}$ or $3^{\text {rd }}$ quartiles for either PCPs or MHPs) and 3) low (states ranked on $1^{\text {st }}$ quartile for PCPs and MHPs). 


\section{Statistical analysis}

TMH adoption rates were calculated for all mental health facilities and for specific subgroups of $\mathrm{MH}$ facilities categorized by facility and state-level characteristics (environmental resource, policy and, market characteristics). The adjusted association of each explanatory variable and TMH adoption was examined with multi-level logistic regressions because facilities (Level-1) were nested within states (Level-2). The level-1 variables included all the facility characteristics, and the level-2 variables included the state-level environmental resource, telehealth policy environment, and market characteristics. Wald chi-square tests were used to evaluate state variance in TMH adoption. Variance Partition Coefficients (VPCs) were calculated to quantify variance due to differences between states. Several 2-level logistic regressions were constructed to identify significant factors associated with TMH adoption. The model-building process is outlined in Table 1. Model 1 was an unconditional model where only the intercept was fitted. Model 2 was a random intercept model where all the level-1 explanatory variables were fitted, whereas Models 3 - 5 had level-2 variables added stepwise. The likelihood-ratio (LR) test was used to assess the significance of 2-level models versus (vs.) 1-level models. Data management and analyses were conducted using SAS 9.4 (SAS Institute Inc., Cary, NC) and Stata 14 (StataCorp LLC, College Station, TX).

\subsection{Results}

\section{Description of mental health facilities}

As illustrated in Table 1, most facilities were outpatient settings (84.8\%), were private non-profit (64.0\%), did not have religious affiliations (93.3\%), and had high patient volume (54.6\%, >100 patients daily). Most focused on mental health only (67.5\%), offered comprehensive mental health treatments $(56.1 \%-83.4 \%)$, implemented four or more QI practices 
(71.2\%), or had moderate to high IT capabilities (78.9\%). Most facilities had Medicaid (88.5\%), Medicare (68.3\%), private insurances $(80.0 \%)$ in their payer-mix. The patient mix in most facilities included at least some elderly (64.9\%) and racial/ethnic minority (i.e., African American, Hispanic/Latino, Asian, American Indian, Pacific Islanders) patients (95.2\%); highneed patients such as veterans and patients with MHSA comprised less than $50 \%$ in most facilities (54.3\%-77.5\%). On the state level, most facilities were in states that had grade A/B telehealth reimbursement and coverage (82.4\%), needed patient consent for telehealth services (68.4\%), and required some forms of licenses for cross-state telehealth practice (62.4\%). More than one-third of facilities were in states with a high shortage of PCPs and MHPs; almost twofifths were in states with high percentages (i.e., > 20\%) of rural counties. About half of the facilities were in states with poor broadband access.

\section{Description of TMH adoption among mental health facilities}

Overall, $25.9 \%$ of mental health facilities reported adopting TMH as one of their treatment approaches. The adoption of TMH varied widely by facility-level characteristics, ranging from $10.7 \%$ in residential facilities to $92.6 \%$ in Veteran Administration Health Centers (VAHCs) (Table 2). TMH adoption rates were highest in Veteran facilities (87.7\%), facilities with higher treatment capacities $(28.7 \%-83.1 \%)$, and those with high IT capacities (55.0\%). At the state level, the adoption rates varied from as low as $3.4 \%$ in Connecticut to as high as $68.8 \%$ in North Dakota. Adoption rates varied by environmental resource, policy and market characteristics. Adoption rates (42.1\%) were the highest in states with high broadband access, and the lowest (16.1\%) in states with low levels of provider shortages (Table 2).

\section{Facility-level factors associated with TMH adoption}


Results from the fully adjusted analyses (Model 5) indicated that facility characteristics in all five domains (facility type, comprehensiveness of mental health treatment, QI practice, IT capability, and payer and case-mix) were significantly associated with TMH adoption (Table 3). It is noteworthy that Veteran Administration Health Centers were 18 times more likely to adopt $\mathrm{TMH}$ as psychiatric inpatient facilities [Adjusted Odds Ratio $(\mathrm{AOR})=18.53,95 \%$ Confidence Interval (95\%CI): 10.66-32.21]. Facilities with high IT capacities were almost three times more likely as those with low capacities to have TMH (AOR $=2.89,95 \% \mathrm{CI}$ : 2.10-3.98). Other facility characteristics associated with higher likelihood of TMH adoption included: inclusion of outpatient settings; high patient volume; public ownership; availability of comprehensive MH treatment; use of QI practices; private or non-Medicaid public payers; and treating elderly patients or higher percentages of veterans (AORs: $1.16-2.41$ ). On the other hand, facilities that were affiliated with religious organizations [AOR (95\%CI): $0.72(0.57-0.91)]$ or treated higher percentages of African American patients [AOR (95\%CI): 0.74 (0.65-0.85)] or patients with MHSA (AORs: $0.81-0.83$ ) were significantly less likely to have adopted TMH (Table 3).

\section{State-level factors associated with TMH adoption}

As presented in Table 1, results of Wald Chi-square tests indicated significant variation in TMH adoption across states. Adding environmental, policy, and market characteristics (level-2 variables) into the model (Model 5) reduced the proportion of variance in TMH adoption due to state variance from $15.9 \%$ to $6.5 \%$ (Table 1). Market characteristics contributed most to the variance reduction (14.1\% in Model 3 to $7.0 \%$ in Model 4). In the fully adjusted model (Table 3), licensure policy and rurality were significantly associated with TMH adoption. Facilities located in states having a special application process for licenses used in cross-state telehealth practice were more than twice as likely to have adopted TMH as compared to those located in 
states not requiring or defining such license $[\mathrm{AOR}=2.24,95 \%$ Confidence Interval $(95 \% \mathrm{CI})$ : $1.16-4.32]$. The odds of TMH adoption across states increased with increasing percentage of rural counties (AORs: 2.09-2.36, $\mathrm{p}<0.01$ ). Other policy factors, provider shortages, and broadband access were not associated with TMH adoption.

\subsection{Discussion}

In this large population-based study of U.S. mental health facilities, only $26 \%$ reported using TMH as a treatment approach in 2016. This adoption rate was much lower than the rate reported for general telehealth (42\%) in acute care hospitals (American Hospital Association Annual Survey of Hospitals), ${ }^{39}$ but consistent with findings from the American Medical Association's 2016 Physician Practice Benchmark Survey indicating that $27.8 \%$ of psychiatrists had used telemedicine to interact with patients. ${ }^{85}$ One reason for the discrepancy in adoption rates may be the high need in acute care hospitals for telehealth services such as teleradiology, teledermatology, teleophthalmology, and telecardiology. ${ }^{86,87}$

Our results indicated that the adoption of TMH by a mental health facility could be influenced by multiple factors, including the type of facility, $\mathrm{MH}$ treatment capacities, IT capability, QI practice, and payer and case-mix of the facility. For example, TMH adoption was significantly higher in Veteran Administration Medical Centers and facilities that had higher proportions of veteran patients. As the nation's largest health care system, the VA has been the leader in telehealth in the US since $2003 .{ }^{88}$ In addition to the VA's continuous commitment to telehealth and mental health, the integrated nature of the system may also contribute to the adoption of TMH. ${ }^{39,89}$ Mental health facilities that included outpatient settings were also more likely to have $\mathrm{TMH}$, likely reflecting the fact that most $\mathrm{MH}$ services adapted to $\mathrm{TMH}$ are provided in outpatient settings. ${ }^{28,52}$ Consistent with previous reports, ${ }^{39,69,70}$ ownership was also 
strongly associated with TMH adoption. In addition, public mental facilities were more likely to adopt TMH as compared to private for-profit facilities, perhaps due to differences in fiscal incentives. ${ }^{90,91}$ Given that most mental health facilities were private non-profit, future studies should explore barriers to TMH adoption among non-profit facilities to invest in TMH.

In addition to facility type, higher IT capacity was associated with higher TMH adoption. This association may in part reflect differences in infrastructure. For example, facilities with higher IT capacities are likely those with a patient referral, health records, and/or provider collaboration system (i.e., part of the definition of IT capacity in our study). These systems are often required for effective and sustainable TMH implementation. ${ }^{92}$ TMH adoption was also more likely among facilities with comprehensive $\mathrm{MH}$ treatments. It is plausible that facilities that adopted TMH can get access to remote specialists, thereby offering a variety of $\mathrm{MH}$ services. As this was a cross-sectional study, we are unable to parse out whether TMH led to comprehensive services.

Facility payer type and patient-mix were also significantly associated with TMH adoption. Facilities treating patients insured by Medicare, VHA, and private payers were more likely to adopt TMH. Facilities treating elderly patients were more likely to have TMH as compared to those who did not. The often complex health care needs of elderly patients with mental health conditions may contribute to TMH adoption, as TMH may facilitate the integrated management of mental and physical chronic conditions. ${ }^{93}$ In contrast, TMH adoption was significantly less likely among facilities serving higher percentages of African Americans, possibly due to lack of trust in new health care innovations among this group. ${ }^{94}$ George and colleague reported that African Americans showed more concerns about confidentiality, privacy, and care quality of telemedicine as compared to Hispanic controls. ${ }^{95}$ As African Americans tend 
to experience more severe mental health conditions due to access barriers, ${ }^{96}$ demonstration and educational programs are needed to increase the "buy-in" of TMH among African American patients.

Facilities that primarily served patients with co-occurring mental health and substance abuse disorders were significantly less likely to adopt TMH relative to those serving fewer MHSA patients. TMH has been considered a promising tool to combat the U.S. substance abuse crisis by improving access to substance abuse experts and mental health specialists. ${ }^{97}$ However, TMH benefits may be limited due to the legal restriction on prescribing Medication-Assisted Treatments via TMH. ${ }^{98}$ Policy initiatives such as the Improving Access to Remote Behavioral Health Treatment Act of 2018, which aims to boost availability of Medication-Assisted Treatment prescribers and ease legal restrictions, may help fully realize the potential of $\mathrm{TMH}$ in behavioral health. ${ }^{98}$

TMH adoption rates varied substantially across states, and the variation was primarily driven by differences in telehealth policies and level of rurality. TMH adoption was more likely in states with more rural counties. This finding is consistent with the previous report on telehealth adoption among acute hospitals. The higher telehealth/TMH adoption in rural areas might be a result of financial incentives provided by federal and foundation funding that focuses on rural health. ${ }^{39,99,100}$ Mental health facilities located in states that had a special application process for licensure for providing interstate telehealth services were more likely to have TMH, than those in states without specific licensure requirements. As of 2017, medical boards in nine states had established processes to issue telehealth-specific licenses to allow out-of-state providers to provide telehealth services. ${ }^{77}$ Twenty-two states had adopted the Federation of State Medical Board's Interstate Medical Licensure Compact to allow an expedited process of 
applying for licenses in other states. ${ }^{77}$ Although adopting the Compact policy was not associated with TMH adoption in our results, it could nonetheless help increase future TMH adoption, as more states adopt this policy and facilities acclimatize themselves to this new policy. In contrast to the observed associations of TMH adoption to state licensure policies, telehealth reimbursement and consent policy were unrelated to TMH adoption, likely reflecting the improvement in overall telehealth coverage and reimbursement in recent years. ${ }^{76}$

This is the first study to our knowledge to investigate TMH adoption rates and associated multilevel factors among U.S. mental health facilities. Additional strengths include the large, representative sample and the use of comprehensive linked data from all 50 states and the District of Columbia. Our findings not only provide robust estimates of overall TMH adoption rates in U.S. mental health facilities but also offer insight into factors that may influence facilitylevel decisions to invest in TMH and suggest potential strategies to increase adoption. However, several limitations should be noted. First, we estimated TMH adoption based on the reported telemedicine use among mental health facilities, and our data did not allow determination of how telemedicine was used. Thus, we may have over-estimated TMH adoption and use. Second, limited data availability only allowed us to measure environmental resources and market characteristics at the state level; thus we were unable to capture within-state variation in these factors. In addition, we were not able to capture the financial profile of mental health facilities or potential market competition, which could also affect the decision to invest in TMH.

Furthermore, N-MHSS did not adjust for non-response; the results of our study may not be generalizable to facilities (9.9\%) that did not participate in the 2016 N-MHSS. Finally, as the study relied on cross-sectional data, relationships should be considered associative rather than causal. 
In summary, although TMH appears to offer a promising tool for facilities to expand access to care and serve high-need patients, only one in four mental health facilities adopted TMH in 2016. TMH adoption was affected by multiple facility and state-level factors. The legal or regulatory burden on providers in seeking licensure for cross-state practice and low facility IT capacity may discourage TMH adoption. Racial disparities in $\mathrm{TMH}$ access warrant further attention. Policy initiatives that could facilitate the prescribing of substance use disorder medications via TMH are needed to increase access to care for patients with substance use disorders.

\subsection{Declaration of Conflicting Interests and Funding Source}

The authors declare that there is no conflict of interest. Research reported in this was supported by the NIGMS of the National Institutes of Health under award number U54GM104942. The content is solely the responsibility of the authors and does not necessarily represent the official views of the National Institutes of Health. 


\section{Table 2.1 Model building process for multi-level multivariable logistic regressions on Telemental health (TMH) adoption among mental health facilities in the United States.}

\begin{tabular}{|c|c|c|c|c|c|}
\hline & Model 1 & Model 2 & Model 3 & Model 4 & Model 5 \\
\hline Model description & $\begin{array}{c}\text { Unconditional } \\
\text { random } \\
\text { intercept } \\
\text { model with } \\
\text { state }\end{array}$ & $\begin{array}{c}\text { level-1 } \\
\text { variables }^{\text {a }}\end{array}$ & $\begin{array}{c}\text { level-1 } \\
\text { variables a \& } \\
\text { level-2 } \\
\text { variables } \\
\text { (policy) }\end{array}$ & $\begin{array}{c}\text { level-1 } \\
\text { variables }{ }^{\text {a }} \& \\
\text { level-2 } \\
\text { variables } \\
\text { (policy \& } \\
\text { market) }^{\mathrm{b}}\end{array}$ & $\begin{array}{c}\text { level-1 } \\
\text { variables }{ }^{\mathrm{a}} \& \\
\text { level-2 } \\
\text { variables } \\
\text { (policy \& } \\
\text { market \& } \\
\text { environmental } \\
\text { resource) }^{\mathrm{b}} \\
\end{array}$ \\
\hline $\begin{array}{l}\text { Level-2 residual variance } \\
\text { (Standard error) }\end{array}$ & $0.584(0.129)$ & $0.624(0.141)$ & $0.541(0.124)$ & $0.249(0.062)$ & $0.230(0.059)$ \\
\hline Wald Chi-square $^{d}$ & $4.527^{*}$ & $4.425^{*}$ & $4.363^{*}$ & $4.016^{*}$ & $3.905^{*}$ \\
\hline VPC $^{\text {e }}$ & 0.151 & 0.159 & 0.141 & 0.070 & 0.065 \\
\hline Model fit (-2LL) ${ }^{f}$ & $898.73^{\text {*** }}$ & $714.00^{\text {**** }}$ & $542.39^{* * *}$ & $266.24^{* * *}$ & $233.00^{* * *}$ \\
\hline
\end{tabular}

Note: The study population included mental health facilities located in the 50 states and the District of Columbia that responded to the query about telemedicine use and patient characteristics in the 2016 N-MHSS. Facilities in American Samoa, Guam, Puerto Rico, and Virgin Islands were excluded due to insufficient information on telehealth policies.

a level-1 variables included all facility characteristics: the type of facility setting, ownership, religious affiliation; the number of mental health treatment approaches, mental health services, mental health programs, as well as quality improvement practice, the availability of non-English service, the IT capacity, the acceptance of Medicare, Medicaid, or Veteran Health Administration patient, licensing, certification, or accreditation of the facility, average daily number of patients, the number of mental health admissions in last year, the percentage of veteran patients in last year, and daily patient composition [e.g., \% cooccurring mental health and substance abuse patients, $\%$ female, $\%$ elderly (> 65 years old), $\%$ Hispanic/Latino, $\%$ African American, \% Minority (American Indian/Asian/Pacific Islanders)].

${ }^{\mathrm{b}}$ level-2 variables included environmental-, policy-, and market characteristics measured at the state-level: \%counties with broadband access, the American Telemedicine Association telehealth coverage \& reimbursement grading, patient consent policy, interstate telehealth licensure policy, and \% rural counties.

${ }^{c}$ the random slope model included all the variables in Model 3 as fixed-effects and $\%$ female patients per day as randomeffects.

${ }^{\mathrm{d}}$ Wald Chi-square values were calculated as (level-2 residual variance/standard error), compared against the critical value of Chi-square with 1 degree of freedom (3.841).

e The Variance Partition Coefficient (VPC) was calculated with the formula: level-2 residual variance / (level-1 residual variance + level-2 residual variance), where level-1 residual variance for logistic model $=3.29$.

${ }^{\mathrm{f}}$ Model fit was assessed by the Likelihood ratio test $=$ Log likelihood from 2-level model - Log likelihood from single-level model. A significant Likelihood ratio test indicates the necessity of a 2-level model.

*: $\mathrm{p}<0.05 ;{ }^{* * *}: \mathrm{p}<0.001$. 
Table 2.2 Descriptions of mental health facilities and Telemental health (TMH) adoption among mental health facilities in the United States.

\begin{tabular}{|c|c|c|c|c|}
\hline & \multicolumn{2}{|c|}{ All facilities } & \multicolumn{2}{|c|}{ Facilities with TMH } \\
\hline & $\mathbf{N}$ & Column \% & $\mathbf{N}$ & $\%$ \\
\hline Total & 11,883 & 100.0 & 3,082 & 25.9 \\
\hline \multicolumn{5}{|c|}{ Level-1: Facility characteristics } \\
\hline \multicolumn{5}{|c|}{ Type of facility } \\
\hline \multicolumn{5}{|l|}{ Care setting } \\
\hline Psychiatric inpatient & 1,804 & 15.2 & 356 & 19.7 \\
\hline Residential & 1,633 & 13.7 & 174 & 10.7 \\
\hline VAMC & 377 & 3.2 & 349 & 92.6 \\
\hline $\mathrm{CMHC}$ & 2,587 & 21.8 & 1,079 & 41.7 \\
\hline Outpatient & 5,088 & 42.8 & 1,030 & 20.2 \\
\hline Multi-setting & 394 & 3.3 & 94 & 23.9 \\
\hline \multicolumn{5}{|l|}{ Ownership } \\
\hline Private-for-profit & 2,069 & 17.4 & 380 & 18.4 \\
\hline Private-non-profit & 7,601 & 64.0 & 1,714 & 22.5 \\
\hline Public & 2,213 & 18.6 & 988 & 44.6 \\
\hline \multicolumn{5}{|l|}{ Religious affiliation } \\
\hline Yes & 763 & 6.4 & 105 & 13.8 \\
\hline No & 11,091 & 93.3 & 2,965 & 26.7 \\
\hline Not reported & 29 & 0.2 & 12 & 41.4 \\
\hline \multicolumn{5}{|c|}{ Annual mental health admissions } \\
\hline $0-100$ & 3,323 & 28.0 & 549 & 16.5 \\
\hline $100-250$ & 1,472 & 12.4 & 329 & 22.4 \\
\hline $250-500$ & 1,474 & 12.4 & 395 & 26.8 \\
\hline $500-1000$ & 3,684 & 31.0 & 1,192 & 32.4 \\
\hline$>1000$ & 1,930 & 16.2 & 617 & 32.0 \\
\hline \multicolumn{5}{|l|}{ Daily volume of patients a } \\
\hline $1-100$ & 5,398 & 45.4 & 938 & 17.4 \\
\hline $100-250$ & 4,340 & 36.5 & 1,320 & 30.4 \\
\hline $250-500$ & 1,311 & 11.0 & 488 & 37.2 \\
\hline $500-1000$ & 388 & 3.3 & 123 & 31.7 \\
\hline$>1000$ & 446 & 3.8 & 213 & 47.8 \\
\hline
\end{tabular}

Comprehensiveness of mental health treatments

\section{Treatment focus}

Mental health only

Mental health \& Substance Abuse

General health

\# of mental health treatment approaches ${ }^{b}$

$0-3$
8,019

3,499

365

1,967
67.5

29.4

3.1

16.6

237
20.7

34.8

$203 \quad 55.6$

12.0 
$\begin{array}{llllll}4-5 & 9,916 & 83.4 & 2,845 & 28.7\end{array}$

\# of mental health services ${ }^{\mathrm{c}, \mathrm{l}}$

$\begin{array}{lllll}0-4 & 6,668 & 56.1 & 1,128 & 16.9\end{array}$

$\begin{array}{lllll}\geq 5 & 5,212 & 43.9 & 1,954 & 83.1\end{array}$

\# of special mental care programs ${ }^{\mathrm{d}}$

$\begin{array}{lcccc}0-3 & 10,541 & 88.7 & 2,538 & 24.1 \\ 4-5 & 1,342 & 11.3 & 544 & 75.9\end{array}$

Non-English services

\begin{tabular}{|c|c|c|c|c|}
\hline Yes & 7,197 & 60.6 & 2,064 & 28.7 \\
\hline No & 4,685 & 39.4 & 1,018 & 21.7 \\
\hline \multicolumn{5}{|c|}{ Quality improvement practice $^{e}$} \\
\hline $0-3$ & 3,377 & 28.4 & 696 & 20.6 \\
\hline 4 & 3,708 & 31.2 & 915 & 24.7 \\
\hline 5 & 4,757 & 40.0 & 1,462 & 30.7 \\
\hline \multicolumn{5}{|c|}{ IT capacity $^{f, l}$} \\
\hline Low & 2,509 & 21.1 & 310 & 12.4 \\
\hline Moderate & 9,006 & 75.8 & 2,577 & 28.6 \\
\hline High & 342 & 2.9 & 188 & 55.0 \\
\hline
\end{tabular}

Medicaid

$\begin{array}{lllll}\text { Yes } & 10,515 & 88.5 & 2,635 & 25.1\end{array}$

$\begin{array}{lllll}\text { No } & 1,155 & 9.7 & 334 & 28.9\end{array}$

$\begin{array}{lllll}\text { Not reported } & 213 & 1.8 & 113 & 53.1\end{array}$

Medicare

Yes

No

$\begin{array}{cccc}8,120 & 68.3 & 2,339 & 28.8 \\ 3,454 & 29.1 & 630 & 18.2 \\ 309 & 2.6 & 113 & 36.6\end{array}$

Not reported

Veteran Health Administration

$\begin{array}{lcccr}\text { Yes } & 2,613 & 22.0 & 1,131 & 43.3 \\ \text { No } & 6,770 & 57.0 & 1,350 & 19.9 \\ \text { Not reported } & 2,500 & 21.0 & 601 & 24.0\end{array}$

Any private insurance

\begin{tabular}{lcccc} 
Yes & 9,501 & 80.0 & 2,694 & 28.4 \\
No & 2,158 & 18.2 & 306 & 14.2 \\
Not reported & 224 & 1.9 & 82 & 36.6 \\
\hline
\end{tabular}

Any elderly a

$\begin{array}{lcccr}\text { Yes } & 7,714 & 64.9 & 2,372 & 30.7 \\ \text { No } & 4,169 & 35.1 & 710 & 17.0 \\ \text { Females a } & & & & \\ 0-10 \% & 567 & 4.8 & 199 & 35.1 \\ 11-50 \% & 5,872 & 49.4 & 1,399 & 23.8\end{array}$




$$
>50 \%
$$

\% African American a

$$
\begin{aligned}
& 0-20 \% \\
& >20 \%
\end{aligned}
$$

Any Hispanic/Latino patients ${ }^{\text {a }}$

Yes

No

Any minority patients a

Yes

No

\% Mental health \& substance abuse patients ${ }^{\text {a }}$

$0-20 \%$

$20-40 \%$

$40-60 \%$

$60-80 \%$

Not reported

Annual \% of veteran patients

$0-50 \%$

$>50 \%$

Not reported
5,444

9,068

2,815

5,993

5,890

11,308

575

4,327

2,127

1,256

1,536

2,637

9,209

302

2,372
45.8

76.3

23.7

2,520

562

27.8

20.0

27.0

24.9

$49.6 \quad 1,465$

95.2

2,895

25.6

4.8

187

32.5

21.6

28.7

27.4

23.0

31.9

22.2

354

840

22.0

87.7

2.5

2,030

33.2

Level-2 variables: Policy characteristics ${ }^{\mathrm{g}}$

ATA telehealth coverage $\&$ reimbursement grading ${ }^{\text {h }}$

$\begin{array}{lcccc}\text { A } & 1,362 & 11.5 & 427 & 31.4 \\ \text { B } & 8,425 & 70.9 & 2,098 & 24.9 \\ \text { C } & 2,096 & 17.6 & 557 & 26.6\end{array}$

Patient consent ${ }^{i}$

$\begin{array}{lllll}\text { Yes } & 8,127 & 68.4 & 2,058 & 25.3 \\ \text { No } & 3,756 & 31.6 & 1,024 & 27.3\end{array}$

Licensure $^{\mathrm{i}}$

Individual state

1,398

11.8

447

32.0

FSMB Compact

2,670

22.5

707

26.5

Special telehealth

1,450

12.2

485

33.4

With exemptions

1,900

16.0

641

33.7

Not required/defined

4,465

37.6

802

18.0

\section{Level-2 variables: Market characteristics ${ }^{\text {h }}$}

\section{Provider shortage $^{\mathrm{j}}$}

Low

Moderate

High

$\%$ rural counties $^{j}$
4,215

3,133

4,535

6,114

$0-10 \%$

35.5

26.4

146

16.1

1,478

27.1

38.2

1,458

34.3 


\begin{tabular}{|c|c|c|c|c|}
\hline $10-20 \%$ & 3,510 & 29.5 & 1,172 & 33.4 \\
\hline$\geq 20 \%$ & 2,259 & 19.0 & 813 & 36.0 \\
\hline \multicolumn{5}{|c|}{ Level-2 variables: Environmental resources ${ }^{h}$} \\
\hline \multicolumn{5}{|c|}{ \% counties with broadband access ${ }^{k}$} \\
\hline $0-10 \%$ & 6,112 & 51.4 & 1,082 & 17.7 \\
\hline $10-20 \%$ & 4,391 & 37.0 & 1,419 & 32.3 \\
\hline$\geq 20 \%$ & 1,380 & 11.6 & 581 & 42.1 \\
\hline
\end{tabular}

Note: The study population included mental health facilities located in the 50 states and the District of Columbia that responded to the query about telemedicine use and patient characteristics in the $2016 \mathrm{~N}$-MHSS. Facilities in American Samoa, Guam, Puerto Rico, and Virgin Island were excluded due to insufficient information on telehealth policies.

a N-MHSS measured total number of patients and patient mix of a facility on a specific day (April 29, 2016).

b Mental health treatment approaches evaluated in N-MHSS included individual psychotherapy, couples/family/group therapy, cognitive behavioral/dialectical behavioral therapy/behavior modification, integrated dual disorders treatment, trauma therapy, activity therapy, electroconvulsive therapy, and psychotropic medication. c Mental health services evaluated in N-MHSS included intensive case/case management/chronic disease/illness management, integrated primary care services, any counseling, family psychoeducation, education services, psychosocial rehabilitation services, psychiatric emergency walk-in services, suicide prevention services, peer support services, screening for tobacco use, smoking cessation services.

d Special mental health programs included mental health programs that is dedicated or designed exclusively for serious mental illnesses, co-occurring mental and substance abuse disorders, post-traumatic stress disorder, senior/elderly patients or veterans.

e Quality improvement practice evaluated in N-MHSS included continuing education, case review, outcome followup after discharge, utilization review, and satisfaction surveys.

f The IT capacity was defined as the level of technology involvement in 14 routine facility activities, including mental health intake, scheduling appointments, assessment/evaluation, treatment planning, client progress monitoring, discharge, referral, issue/receive lab results, prescribing/dispensing medications, checking medication interactions, health records, provider collaboration, billing, and satisfaction surveys. The level of technology involvement was measured by the mean score of all activities rated on a 3-point scale, where "1" denotes no technology involvement (i.e., rely on paper), "2" indicates partial involvement (i.e., both electronic and paper), and "3" represents fully involvement (i.e., only electronic).

g environmental-, policy-, and market characteristics were measured for the state where the facility was located. $\mathrm{h}$ the American Telemedicine Association (ATA) has given each state a grade (A, B, C, or F) for the coverage and reimbursement policies for $\mathrm{TH}$, basing on health plan parity and Medicaid conditions of payment. $i$ the patient consent policy that requires informed consent before telehealth services and the licensure policy that requires license for interstate telehealth practice were obtained from the Centers for Connected Health Policy's (CCHP) annual State Telehealth Laws and Reimbursement Policies Report.

$\mathrm{j}$ The percentage counties with primary care physician (PCP) shortage, mental health professional (MHP) shortage, and the percentage of rural counties were obtained from the Area Health Resources File (AHRF).

$\mathrm{k}$ Broadband access was defined as having access to $25 \mathrm{Mbps} / 3 \mathrm{Mbps}$ service; \% counties with broadband access in the locating state were derived from the 2016 Broadband Progress Report.

1 Missing data category is not presented due to small sample size $(\mathrm{n}<10)$. 
Table 2.3 Adjusted odds ratios (AORs) and 95\% confidence intervals (95\% CI) of facility- and state-level characteristics on Telemental health (TMH) adoption among mental health facilities in the United States.

\begin{tabular}{|c|c|c|c|}
\hline Variables & Model 2 & Model 4 & Model 5 \\
\hline & AOR $[95 \% \mathrm{CI}]$ & AOR $[95 \% \mathrm{CI}]$ & AOR $[95 \% \mathrm{CI}]$ \\
\hline \multicolumn{4}{|c|}{ Level-1: Facility characteristics } \\
\hline \multicolumn{4}{|c|}{$\underline{\text { Type of facility }}$} \\
\hline \multicolumn{4}{|l|}{ Care setting } \\
\hline Residential & $1.27[0.98,1.63]$ & $1.27[0.99,1.64]$ & $1.27[0.99,1.64]$ \\
\hline VAMC & $18.30[10.52,31.82]^{* * *}$ & $18.38[10.57,31.97]^{* * *}$ & $18.53[10.66,32.21]^{* * *}$ \\
\hline СMHC & $2.46[2.03,2.98]^{* * *}$ & $2.46[2.03,2.98]^{* * *}$ & $2.41[1.99,2.93]^{* * *}$ \\
\hline Outpatient & $1.57[1.30,1.89]^{* * *}$ & $1.57[1.31,1.89]^{* * *}$ & $1.56[1.30,1.88]^{* * *}$ \\
\hline Multi-setting & $1.94[1.43,2.62]^{* * *}$ & $1.94[1.43,2.63]^{* * *}$ & $1.94[1.43,2.63]^{* * *}$ \\
\hline Psychiatric inpatient & & Reference & \\
\hline \multicolumn{4}{|l|}{ Ownership } \\
\hline Private-non-profit & $1.10[0.94,1.29]$ & $1.11[0.95,1.29]$ & $1.10[0.95,1.29]$ \\
\hline Public & $1.67[1.39,2.01]^{* * *}$ & $1.67[1.39,2.01]^{* * *}$ & $1.66[1.38,2.00]^{* * *}$ \\
\hline Private-for-profit & & Reference & \\
\hline \multicolumn{4}{|l|}{ Religious affiliation } \\
\hline Yes & $0.72[0.57,0.91]^{* *}$ & $0.72[0.57,0.91]^{* *}$ & $0.72[0.57,0.91]^{* *}$ \\
\hline No & & Reference & \\
\hline \multicolumn{4}{|c|}{ Annual mental health admissions } \\
\hline $100-250$ & $1.10[0.92,1.32]$ & $1.10[0.92,1.32]$ & $1.10[0.92,1.32]$ \\
\hline $250-500$ & $1.27[1.05,1.52]^{*}$ & $1.27[1.05,1.52]^{*}$ & $1.26[1.05,1.52]^{*}$ \\
\hline $500-1000$ & $1.33[1.12,1.59]^{* *}$ & $1.33[1.12,1.58]^{* *}$ & $1.33[1.12,1.59]^{* *}$ \\
\hline$>1000$ & $1.39[1.15,1.67]^{* *}$ & $1.39[1.15,1.67]^{* *}$ & $1.39[1.15,1.68]^{* *}$ \\
\hline $0-100$ & & Reference & \\
\hline \multicolumn{4}{|l|}{ Total patients ${ }^{\text {a }}$} \\
\hline $100-250$ & $1.11[0.97,1.27]$ & $1.11[0.97,1.27]$ & $1.12[0.98,1.28]$ \\
\hline $250-500$ & $1.10[0.91,1.32]$ & $1.09[0.91,1.32]$ & $1.11[0.92,1.33]$ \\
\hline $500-1000$ & $0.86[0.64,1.15]$ & $0.86[0.64,1.14]$ & $0.87[0.65,1.16]$ \\
\hline$>1000$ & $1.41[1.08,1.83]^{*}$ & $1.41[1.08,1.83]^{*}$ & $1.42[1.09,1.84]^{*}$ \\
\hline $1-100$ & & Reference & \\
\hline
\end{tabular}

Comprehensiveness of mental health treatments

Treatment focus

Mental health \&

Substance Abuse

General services

$1.24[1.10,1.39]^{* * *}$

$1.24[1.10,1.39]^{* * *}$

$1.23[1.10,1.38]^{* * *}$

Mental health only

$1.38[1.00,1.90]^{*}$

$1.38[1.01,1.90]^{*}$

$1.38[1.01,1.90]^{*}$

\# of mental health treatment approaches ${ }^{b}$

4-5

$2.00[1.69,2.37]^{* * *}$

$2.00[1.69,2.37]^{* * *}$

$2.00[1.69,2.36]^{* * *}$ 
0-3

\# of mental health services ${ }^{\mathrm{c}}$

Reference

\begin{tabular}{|c|c|c|c|}
\hline$\geq 5$ & $1.80[1.61,2.00]^{* * *}$ & $1.80[1.61,2.00]^{* * *}$ & $1.79[1.61,2.00]^{* * *}$ \\
\hline $0-4$ & & Reference & \\
\hline
\end{tabular}

\# of special mental care programs ${ }^{\mathrm{d}}$
4-5
$1.15[0.99,1.34]$
$1.15[0.99,1.34]$
$1.15[0.99,1.34]$
$0-3$
Reference

Non-English services

Yes

$1.20[1.07,1.35]^{* *}$

$1.20[1.08,1.35]^{* *}$

$1.21[1.08,1.36]^{* *}$

No

Reference

\section{Quality improvement practice}

\# of Quality Improvement practice ${ }^{\mathrm{e}}$

\begin{tabular}{|c|c|c|c|}
\hline 4 & $1.13[0.99,1.29]$ & $1.13[0.99,1.29]$ & $1.13[0.99,1.29]$ \\
\hline 5 & $1.35[1.19,1.53]^{* * *}$ & $1.35[1.19,1.53]^{* * *}$ & $1.35[1.18,1.53]^{* * *}$ \\
\hline $0-3$ & \multicolumn{3}{|c|}{ Reference } \\
\hline \multicolumn{4}{|c|}{ IT capability $f$} \\
\hline Moderate & $1.91[1.65,2.21]^{* * *}$ & $1.91[1.65,2.22]^{* * *}$ & $1.90[1.64,2.20]^{* * *}$ \\
\hline High & $2.93[2.13,4.03]^{* * *}$ & $2.93[2.13,4.04]^{* * *}$ & $2.89[2.10,3.98]^{* * *}$ \\
\hline Low & \multicolumn{3}{|c|}{ Reference } \\
\hline \multicolumn{4}{|c|}{ Payer mix } \\
\hline \multicolumn{4}{|l|}{ Medicaid } \\
\hline Yes & $1.13[0.91,1.41]$ & $1.13[0.91,1.41]$ & $1.12[0.90,1.40]$ \\
\hline No & & Reference & \\
\hline \multicolumn{4}{|l|}{ Medicare } \\
\hline Yes & $1.25[1.08,1.45]^{* *}$ & $1.25[1.08,1.45]^{* *}$ & $1.26[1.09,1.47]^{* *}$ \\
\hline No & & Reference & \\
\hline \multicolumn{4}{|c|}{ Veteran Health Administration } \\
\hline Yes & $1.37[1.21,1.56]^{* * *}$ & $1.37[1.21,1.56]^{* * *}$ & $1.36[1.20,1.55]^{* * *}$ \\
\hline No & & Reference & \\
\hline \multicolumn{4}{|l|}{ Private } \\
\hline Yes & $1.51[1.27,1.79]^{* * *}$ & $1.50[1.27,1.78]^{* * *}$ & $1.47[1.24,1.74]^{* * *}$ \\
\hline No & & Reference & \\
\hline \multicolumn{4}{|c|}{ Case-mix } \\
\hline \multicolumn{4}{|c|}{$\%$ of female patients ${ }^{a}$} \\
\hline $11-50 \%$ & $0.77[0.57,1.05]$ & $0.77[0.57,1.05]$ & $0.78[0.57,1.06]$ \\
\hline$>50 \%$ & $0.83[0.61,1.13]$ & $0.83[0.61,1.13]$ & $0.83[0.61,1.14]$ \\
\hline $0-10 \%$ & & Reference & \\
\hline \multicolumn{4}{|c|}{ Having elderly patients a } \\
\hline Yes & $1.17[1.03,1.32]^{*}$ & $1.16[1.03,1.32]^{*}$ & $1.16[1.02,1.31]^{*}$ \\
\hline No & & Reference & \\
\hline \multicolumn{4}{|c|}{$\%$ of African American patients ${ }^{a}$} \\
\hline$>20 \%$ & $0.74[0.65,0.85]^{* * *}$ & $0.74[0.65,0.85]^{* * *}$ & $0.74[0.65,0.85]^{* * *}$ \\
\hline
\end{tabular}


$0-20 \%$

Having Hispanic/Latino patients ${ }^{a}$

$\begin{array}{ll}\text { Yes } & 1.09[0.98,1.22] \\ \text { No } & \end{array}$

Any minority patients ${ }^{\text {a }}$

Yes $\quad 1.25[1.00,1.57]$

No

$\%$ of MHSA patients ${ }^{a}$

$\begin{array}{ll}20-40 \% & 1.03[0.89,1.19] \\ 40-60 \% & 0.95[0.80,1.14] \\ >60 \% & 0.83[0.70,0.98]^{*} \\ \text { Not reported } & 0.81[0.67,0.98]^{*} \\ 0-20 \% & \end{array}$

Annual \% of veteran patients

$\begin{array}{ll}>50 \% & 2.74[1.65,4.56]^{* * *} \\ \text { Not reported } & 1.24[1.02,1.50]^{*} \\ 0-50 \% & \end{array}$

Reference
$1.10[0.98,1.23]$

Reference

$$
1.24[0.99,1.56]
$$

$1.24[0.99,1.56]$

Reference

$1.03[0.89,1.19]$

$1.03[0.89,1.19]$

$0.95[0.80,1.14]$

$0.96[0.81,1.14]$

$0.83[0.70,0.98]^{*}$

$0.83[0.70,0.98]^{*}$

$0.81[0.67,0.98]^{*}$

$0.81[0.67,0.98]^{*}$

Reference
$2.75[1.65,4.57]^{* * *}$

$1.24[1.02,1.50]^{*}$

Level-2: Policy characteristics ${ }^{\mathrm{g}}$

ATA telehealth coverage $\&$ reimbursement grading h
A
B
$1.02[0.47,2.21]$
$0.57[0.31,1.05]$
$0.91[0.49,1.68]$
$0.74[0.46,1.19]$
C
Reference

Patient consent ${ }^{\mathrm{i}}$

\begin{tabular}{|c|c|c|c|}
\hline Yes & - & $0.82[0.52,1.29]$ & $0.84[0.61,1.17]$ \\
\hline No & & Reference & \\
\hline
\end{tabular}

\section{Licensure $^{\mathrm{i}}$}

Individual state

FSMB Compact

Special telehealth

With exemptions

Not required/defined

$\begin{array}{ccc}- & 1.70[0.82,3.56] & 1.54[0.88,2.72] \\ - & 1.63[0.93,2.86] & 1.05[0.69,1.59] \\ - & 2.28[1.02,5.06]^{*} & 2.24[1.16,4.32]^{*} \\ - & 1.37[0.73,2.58] & 1.09[0.67,1.78] \\ & \text { Reference } & \end{array}$

Level-2: Market characteristics ${ }^{\mathrm{g}}$

\section{Provider shortage $^{j}$}

High

Moderate

Low

$\%$ rural counties ${ }^{j}$

$10-20 \%$

$\geq 20 \%$

$0-10 \%$

$\begin{array}{lll}- & - & 0.90[0.53,1.54] \\ - & - & 1.41[0.83,2.39]\end{array}$

Reference

$2.09[1.33,3.29]^{* *}$

$2.36[1.34,4.18]^{* *}$

Reference

Level-2: Environmental resources ${ }^{\mathrm{g}}$ 


\section{\% counties with broadband access ${ }^{k}$}

$\begin{array}{lccr}10-20 \% & - & - & 1.02[0.65,1.62] \\ \geq 20 \% & - & - & 1.63[0.92,2.87] \\ 0-10 \% & & \text { Reference } & \end{array}$

Note: The study population included mental health facilities located in the 50 states and the District of Columbia that responded to the query about telemedicine use and patient characteristics in the 2016 N-MHSS. Facilities in American Samoa, Guam, Puerto Rico, and Virgin Islands were excluded due to insufficient information on telehealth policies.

a N-MHSS measured total number of patients and patient mix of a facility on a specific day (April 29, 2016).

b Mental health treatment approaches evaluated in N-MHSS included individual psychotherapy, couples/family/group therapy, cognitive behavioral/dialectical behavioral therapy/behavior modification, integrated dual disorders treatment, trauma therapy, activity therapy, electroconvulsive therapy, and psychotropic medication.

c Mental health services evaluated in N-MHSS included intensive case/case management/chronic disease/illness management, integrated primary care services, any counseling, family psychoeducation, education services, psychosocial rehabilitation services, psychiatric emergency walk-in services, suicide prevention services, peer support services, screening for tobacco use, smoking cessation services.

d Special mental health programs included mental health programs that is dedicated or designed exclusively for serious mental illnesses, co-occurring mental and substance abuse disorders, post-traumatic stress disorder, senior/elderly patients or veterans. e Quality improvement practice evaluated in N-MHSS included continuing education, case review, outcome follow-up after discharge, utilization review, and satisfaction surveys.

f The IT capacity was defined as the level of technology involvement in 14 routine facility activities, including mental health intake, scheduling appointments, assessment/evaluation, treatment planning, client progress monitoring, discharge, referral, issue/receive lab results, prescribing/dispensing medications, checking medication interactions, health records, provider collaboration, billing, and satisfaction surveys. The level of technology involvement was measured by the mean score of all activities rated on a 3-point scale, where "1" denotes no technology involvement (i.e., rely on paper), "2" indicates partial involvement (i.e., both electronic and paper), and "3" represents fully involvement (i.e., only electronic).

g environmental-, policy-, and market characteristics were measured for the state where the facility was located.

$\mathrm{h}$ the American Telemedicine Association (ATA) has given each state a grade (A, B, C, or F) for the coverage and reimbursement policies for $\mathrm{TH}$, basing on health plan parity and Medicaid conditions of payment.

$\mathrm{i}$ the patient consent policy that requires informed consent before telehealth services and the licensure policy that requires license for interstate telehealth practice were obtained from the Centers for Connected Health Policy's (CCHP) annual State Telehealth Laws and Reimbursement Policies Report.

j The percentage counties with primary care physician (PCP) shortage, mental health professional (MHP) shortage, and the percentage of rural counties were obtained from the Area Health Resources File (AHRF).

$\mathrm{k}$ Broadband access was defined as having access to $25 \mathrm{Mbps} / 3 \mathrm{Mbps}$ service; \% counties with broadband access in the locating state were derived from the 2016 Broadband Progress Report.

$* \mathrm{p}<0.05 ; * * \mathrm{p}<0.01 ; * * * \mathrm{p}<0.001$ 


\section{CHAPTER 3}

\section{Telemental Health Use Among Adults with Mental Health Conditions Enrolled in A}

\section{Nationwide Commercial Health Insurance}

\subsection{Abstract}

Objective: To examine telemental health (TMH) use and associated patient and statelevel factors among adults with mental health conditions (MHCs). Data sources: Linked deidentified claims (01/01/2010 - 06/30/2017) from Optum Clinformatics ${ }^{\circledR}$ DataMart, Area Health Resources File, and the National Mental Health Services Survey were used. Study design: This study utilized a retrospective, dynamic cohort of adults with MHCs $(\mathrm{N}=248,060)$. Data collection/methods: MHCs were identified using diagnostic codes, and TMH use was ascertained using procedure codes derived from claims data. Multilevel logistic regression was used to assess the association of TMH use with patient- and state-level factors. Principal findings: Approximately 16 in 10,000 adults with MHCs used TMH. Patient characteristics associated with TMH use included younger age, higher monthly out-of-pocket expenditures, past mental health specialist visits, and diagnosis of a severe MHC (Adjusted Odds Ratio (AOR): 1.42-9.46). TMH use varied widely across states and was significantly and positively associated with high mental health provider shortages and high TMH capacity. Conclusions: TMH use was low among commercially insured MHC patients. TMH use was mainly driven by patients' need, provider shortages, and TMH capacity, suggesting that expanding TMH capacity could help alleviate the unmet need for mental health services.

\subsection{Introduction}

Mental health conditions (MHCs) affect 43.8 million adults in the United States (US). ${ }^{101}$ MHCs can have a devastating impact on all aspects of an individual's life and are the costliest 
health conditions with an annual cost of $\$ 201$ billion on the population level. ${ }^{101,102}$ Despite the availability of effective mental health (MH) treatments, only $43 \%$ of US adults with MHCs received mental health services in $2016 .{ }^{103}$ The longstanding shortage of mental health providers is of a significant contributor to the unmet needs for mental health care, especially in underserved rural areas. ${ }^{104,105}$ It is reported that about $77 \%$ of US counties had a severe shortage of mental health professionals (e.g., psychiatrists, psychologists, and social workers). ${ }^{104}$

The unresolved shortage of mental health providers has incentivized payers and providers to find innovative alternative care delivery models to supplement in-person care. In this context, Telemental health (TMH), broadly defined as the delivery of MH services remotely, has been increasingly considered as a part of mental health care. ${ }^{106}$ TMH has the advantages of expanding patient access to existing providers, decreasing patients' indirect costs (i.e., travel time and cost), and potentially reducing the stigma associated with visiting mental health facilities. ${ }^{30}$ Results from randomized trials have demonstrated that $\mathrm{TMH}$ in various modalities (e.g., internet, phone, video-conferencing) is comparable or even superior to in-person care for evaluating and treating patients with MHCs, including those with severe MHCs, such as schizophrenia. ${ }^{50,51,107}$

Recognizing the potential for TMH to remove access-related barriers to treatment, federal, state, and local governments have made substantial efforts to increase TMH services in recent years. ${ }^{40}$ However, only a few studies have examined TMH use among adults with MHCs in real-world settings using recent data. One study analyzing 2008 and 2009 Medicaid claims reported very low $(<0.1 \%) \mathrm{TMH}$ use and high state-level variations due to differences in coverage and reimbursement policies for TMH across states. ${ }^{41}$ Notable variations in TMH use were also reported in a study analyzing 2004-2014 claims data of Medicare beneficiaries with MHCs, who had the same TMH coverage and reimbursement policies. ${ }^{42}$ While these studies 
illustrated the role of reimbursement policies in TMH use, they also highlighted the wide variation in TMH use among states, despite having the same reimbursement and coverage for TMH services. This finding suggests that patient characteristics and state-level factors may also contribute to TMH use.

Little is known about patient-level factors and other state-level factors associated with TMH use. Previous studies have reported that TMH use was significantly associated with younger age, having any disability, and living in a poor community. ${ }^{43,44}$ Health plan characteristics (e.g., out-of-pocket expenditures ${ }^{45}$ ), factors that may increase the need for TMH/MH services (e.g., previous treatment seeking, ${ }^{46}$ polypharmacy ${ }^{47}$ ), and environmental capacities that allow TMH use were not explored. Furthermore, there is evidence showing that private payers are more likely to be billed for telehealth services than public payers. ${ }^{38}$ While commercial insurance provides an important source of health coverage for adults with MHCs, ${ }^{48}$ knowledge about TMH use among commercially insured adults with MHC is limited. Although TMH has been covered for almost all MH services from various providers (e.g., psychiatrists, social worker, physicians), ${ }^{49}$ how TMH is used in various $\mathrm{MH}$ services and who is delivering TMH in real-world practice remained unknown. Such knowledge is vital to inform health insurers and healthcare policymakers how to better incorporate TMH in delivering mental health services into real-world healthcare settings.

In this study, we examined TMH use, explored the role of TMH use in delivering $\mathrm{MH}$ service, and assessed the association of patient and state-level factors to TMH use in adults with MHCs using linked claims data from a large U.S. commercial plan.

\subsection{Methods}

\section{Data sources}


This study used linked data from several sources: 1) de-identified 10\% sample drawn from the Optum Clinformatics ${ }^{\circledR}$ DataMart (Optum Clinformatics ${ }^{\circledR}$, Eden Prairie, MN, USA) from $1^{\text {st }}$ January 2010 through $30^{\text {th }}$ June $2017 ; 2$ ) the 2016-2017 Area Health Resources File (AHRF); and 3) the 2010 - 2016 National Mental Health Services Survey (N-MHSS). Optum Clinformatics ${ }^{\circledR}$ provides de-identified paid medical and prescription claims and enrollment information for individuals enrolled in a large US commercial health insurance company, which has geographically diverse enrollment across all 50 states and the District of Columbia (DC). ${ }^{108}$ The AHRF includes data on healthcare providers, population characteristics and environment at the county, state and national levels from over 50 data sources. ${ }^{109}$ The N-MHSS is the only source of national and state-level data on mental health service delivery system for all known public and private mental health treatment facilities in the US. ${ }^{78}$ Data from these three sources were linked using state identifiers.

\section{Study design}

This study employed a retrospective, dynamic cohort design with a baseline and followup period. The study cohort consisted of adults with MHC conditions. Adults with any MHC were identified during a calendar year by one inpatient claim or two or more outpatient claims with a primary or secondary diagnosis of schizophrenia, bipolar disorder, major depressive disorder and other MHCs using the International Classification of Diseases, Ninth or Tenth Revision (ICD9/ICD10) codes (ICD9: 291-292, 295 - 316; ICD10: F10-F99). ${ }^{42}$ Study cohorts were identified for each year and pooled together. For adults with MHC who were identified in multiple years, the first observation was kept for subsequent analyses.

TMH use was assessed in the 12 months after first observed MHC diagnosis. For TMH users, the first observed TMH use date was considered as the index date; this date was used to 
define a baseline period. A pseudo-index date was randomly selected from all MH services dates for adults without a record of TMH use. The six months before the index/pseudo-index dates was defined as the baseline period.

\section{Study sample}

The study sample comprised adults at least 18 years of age at baseline) who were: 1) diagnosed with any MHC in a calendar year between 2010 and 2016; 2) had at least two MH service encounters during the study period; and 3) were continuously enrolled in the insurance plan throughout the baseline and 12-month follow-up period. The cohort identification process is illustrated in Figure 3.1. All MH service encounters were identified with procedure codes (Appendix 7.1). ${ }^{42}$

\section{Measures}

Outcome variable: Any TMH Use

Any TMH use was identified from MH service claims using Healthcare Common Procedure Coding System (HCPCS)/ Current Procedural Terminology (CPT) codes (“0188T”, “0189T”, “G0406 - 0408”, “G0425 - 0427”, “G0459”) and/or modifiers appended with the procedure codes (“GT”, “GQ”, “95”). ${ }^{42,49}$ Adults with MHCs with no record of TMH use were considered non-users. We also examined annual TMH visit rate, which was calculated as the number of TMH visits per 1,000 adults with MHCs from 2010 to 2017. The type of MH services delivered via TMH/in-person was determined by HCPCS/CPT codes and modifiers, and the type of provider who delivered TMH/in-person MH services was identified with provider category codes.

Explanatory variables: 
The explanatory variables were selected based on the common and unique constructs from determinants of health model proposed by Wilkinson and Marmot, ${ }^{72}$ the Telehealth Research and Policy Framework proposed by a multidisciplinary group of experts, ${ }^{73}$ and a comprehensive review of the literature. ${ }^{42,110}$ Factors that could affect TMH use can be grouped into several domains, including 1) biological factors (age and sex); 2) health insurance characteristics [types of health insurance (Medicare Advantage/others), types of health plan (Health Maintenance Organization/Preferred Provider Organization/Point of Service/Fee-forservice), average monthly out-of-pocket (OOP) expenditures for medical services]; 3) need factors that may increase the need for TMH [severity of MHCs, number of physical chronic conditions, the presence of polypharmacy (> 5 drug classes in a 90-day period), ${ }^{111}$ obesity, and any substance use disorders (i.e., alcohol, tobacco, drug), and in-person mental health specialist visits]; and 4) environmental factors [state-level poverty, rurality, mental health provider shortages (MHPS), and TMH capacity]. We also adjusted for the year of TMH use to account for policies and other environmental factors that may change over time.

State-level poverty, rurality, and MHPS were measured as the average percentage, within each state, of counties in poverty, rural counties, and counties with MHPS; all percentages were derived using AHRF data. TMH capacity was defined as the average percentage of mental health facilities adopting the use of telemedicine within a state, using $2010-2016$ N-MHSS data. To facilitate interpretation of the relationship between state-level factors and TMH use by patients, we analyzed environmental factors by comparing the lower and upper $50^{\text {th }}$ percentiles.

Patient-level factors were derived using the medical claims, pharmacy claims, and enrollment files from Optum Clinformatics ${ }^{\circledR}$. The severity of MHCs was determined with an algorithm developed by researchers from the Healthcare Cost and Utilization Project. ${ }^{112}$ The 
chronic physical conditions examined were based on a list of 13 chronic conditions (i.e., arthritis, asthma, coronary artery disease, cardiac arrhythmias, congestive heart failure, chronic kidney disease, chronic obstructive pulmonary disease, dementia and related disorders, diabetes, hypertension, hyperlipidemia, osteoporosis, stroke) by the Multiple Chronic Conditions working group. ${ }^{113}$ All the patient-level factors were measured during the baseline period.

\section{Statistical analyses}

The percentage of TMH users were calculated for all adults with MHCs and within subgroups defined by patient-level and state-level characteristics. Several multilevel logistic regressions were constructed to identify factors associated with $\mathrm{TMH}$ use among adults with MHCs, where patients (Level-1) were nested within states (Level-2). Table 2 outlines the modelbuilding process. Level-1 variables included health insurance characteristics as well as biological and need factors, while Level-2 variables included all environmental factors. State-level variations in TMH use were evaluated with Wald chi-square tests and quantified by Variance Partition Coefficients (VPCs). The likelihood-ratio (LR) test was employed to evaluate the significance of two-level models versus (vs.) single-level models. As Medicare Advantage only covered telehealth for those in underserved areas, subgroup analyses were performed for adults with MHCs enrolled in Medicare Advantage plans who lived in underserved states (i.e., states with high level of rurality or shortage of providers). All analyses were conducted using SAS 9.4 (SAS Institute Inc., Cary, NC) and Stata 14 (StataCorp LLC, College Station, TX).

\subsection{Results}

\section{Sample description}

A total of 248,060 adults with MHC were identified. As presented in Table 1, the most prevalent MHC was anxiety disorders (42.5\%). Almost one-third (32.2\%) of the study sample 
had at least one serious MHC, including schizophrenia (1.5\%), bipolar disorder $(7.7 \%)$, and major depressive disorder $(24.8 \%)$. The sample was predominantly female $(61.5 \%)$, with a mean age of 49.2 years [Standard deviation $(\mathrm{SD})=18.4]$.

At baseline, the majority (77.9\%) of the study sample were enrolled in non-HMO plans such as Preferred Provider Organizations, Point of Services, and Fee-for-Services plans. About one-third (32.6\%) of the cohort had received at least one MH service for a severe MHC diagnosis, and $21.9 \%$ had visited a mental health specialist. About $16.9 \%$ had been diagnosed with tobacco/alcohol use disorders, $5.8 \%$ with drug use disorders, and $8.2 \%$ with obesity. Polypharmacy was present in $27.8 \%$ of MHC patients. Nearly two-fifths (38.9\%) of MHC patients were from states with high levels of poverty; the majority were from states with high levels of rurality $(58.2 \%)$ or MHPS $(54.6 \%)$. Over half $(56.5 \%)$ of adults with MHCs in our study sample resided in states with a low TMH capacity (Table 1).

Across states, variation in patient characteristics and environmental factors was substantial. For example, the percentage of adults with severe MHCs varied from $20 \%$ to $45 \%$, and MA-enrollment ranged from $0 \%$ to $94 \%$. Percentages of rural counties and counties with MHPS varied widely, ranging from $0 \%$ to $91.3 \%$ and 0 to $100 \%$, respectively. TMH capacity was as low as $1.8 \%$ in Connecticut and as high as $61.4 \%$ in North Dakota.

\section{Description of MHS delivered by TMH and in-person among TMH users}

Almost all TMH services (95.3\%) were delivered via interactive audio/video telecommunications systems. Across various MHS categories, TMH played an essential role in the delivery of outpatient/inpatient consultation and pharmacological management, with 94.7\% and $53.8 \%$ of these procedures administered via TMH, respectively (Figure 3.2). Among all the MH services delivered by $\mathrm{TMH}$, the most commonly used services were routine office visits 
(40.5\%), initial hospital inpatient care (17.8\%), and pharmacological management (16.6\%). By contrast, the most prevalent in-person MH visits were for psychotherapy (30.5\%). Regarding the type of providers who delivered MHS, most TMH services were delivered by psychiatrists $(62.3 \%)$ and social workers/counselors (21.8\%), whereas in-person MHS were delivered by a range of providers, including psychiatrists (24.5\%), social workers/counselors (21.2\%), family physicians/internalist (11.6\%), and psychologists (11.3\%).

\section{Description of TMH users among adults with MHCs}

From 2010 to 2017, the percentage of TMH users among adults with MHC had increased by 23 -fold [0.03\% to $0.69 \%$ ], and the annual rate of TMH visits had increased by almost eightfold [1.1 to 8.7 visits/1,000 adults with MHCs]. Overall, only $0.16 \%$ of adults with MHC ( $\mathrm{N}=$ 385) had ever used TMH in the 12 months after MHC diagnosis. The percentage of TMH users varied significantly across all patient-level characteristics, except for sex (Table 1). TMH use was highest (1.62\%) among adults with schizophrenia, and lowest (0.03\%) among MHC patients enrolled in non-MA plans. TMH use also varied across states. States with high TMH capacities had the highest TMH use $(0.24 \%)$, whereas states with low MHPS had the lowest use $(0.08 \%)$. A total of 18 states (e.g., Alaska, Connecticut, Delaware) had zero TMH use, most of which were clustered at the Northeast. TMH use was highest in North Dakoda (1.16\%), followed by Tennessee $(0.69 \%)$ and Indiana $(0.68 \%)$.

\section{The association of Patient-level factors with TMH use among adults with MHCs}

Table 2 presents the results of our multilevel logistic regression analyses. In the fully adjusted model (Model 4), age, all insurance characteristics, and most need factors were significantly associated with TMH use. Specifically, age was significantly and inversely associated with TMH use [Adjusted Odds Ratio (AOR) per year increment: 0.98, 95\% 
Confidence Interval (95\%CI): 0.97-0.99]. Furthermore, higher monthly OOP expenditures were associated with higher odds of TMH use [(AORs: 1.48-1.89, $\mathrm{p}<0.01)$ ]. Need factors associated with TMH use included a history of services for a severe MHC diagnosis [AOR (95\%CI): 2.14 (1.66-2.76)], in-person visits to mental health specialists [AOR (95\%CI): 9.46 (7.39-12.11)], and polypharmacy [AOR (95\% CI): $1.42(1.10-1.83)]$ at baseline.

\section{State-level factors associated with TMH use among adults with MHCs}

Results from likelihood ratio tests indicated significant variation in TMH use across states (Table 2). Adding state-level factors to the regression model resulted in a reduction in state-level variance, with VPC values declining from 0.43 to 0.30 ). State-level MHPS and TMH capacity were significantly associated with TMH use: MHC patients residing in states with high levels of MHPS [AOR $(95 \% \mathrm{CI}): 4.65(1.72-12.60)]$ and states with high TMH capacity [AOR (95\%CI): $2.62(1.09-6.28)]$ were more likely to use TMH than were those residing in states with low levels of MHPS and TMH capacity, respectively (Table 2).

\section{Secondary Analysis: Patient- and State-level factors associated with TMH use among MA enrollees with MHC living in underserved states}

As TMH use was much higher in the MA subgroup, we assessed correlates of TMH utilization among MA enrollees in separate analyses. Among 61,861 MA enrollees with MHC lived in underserved states, $0.47 \%$ used TMH. Results from multilevel regressions indicated associations of TMH use to patient- and state-level factors that were similar to those observed in the pooled sample. TMH use was more likely among MA enrollees with MHCs who were younger than 65 years [AOR(95\%CI): 1.99 (1.52-2.60)], who had higher monthly OOP payments (AORs: $1.39-1.57, \mathrm{p}<0.05$ ), who had visited any mental health specialist [AOR (95\% CI): 9.86 (7.40-13.14)], who had received a diagnosis of any severe MHC [AOR (95\%CI): 
1.83 (1.36-2.46)], and who resided in states with high MHPS [AOR (95\%CI): 4.29 (1.79-10.29)]. However, TMH capacities and polypharmacy were not significantly associated with TMH use (Table 3).

\subsection{Discussion}

Overall, our study examined the role of TMH in delivering mental health services and identified patient and state-level factors associated with TMH use in a large commercially insured adult population with MHCs. Approximately 16 in 10,000 adults with MHC and 47 in 10,000 of those enrolled in MA plans and lived in underserved states used TMH in a given year, respectively. We found that most TMH services were delivered by psychiatrists, suggesting TMH may have been used primarily for increasing access to mental healthcare specialists. This may reflect the ongoing severe shortage of psychiatrists and the growing demand for mental healthcare specialists in the US. ${ }^{15,54}$ Given that the American Psychiatric Association (APA) had coordinated with the American Telemedicine Association (ATA) to develop a clinical guideline on videoconferencing in mental health, ${ }^{114}$ it is also plausible that psychiatrists may differ from other mental health providers in training, which leads to a higher uptake of TMH in their practice. Future efforts to expand TMH services may target allied mental health professionals such as psychologists, social workers, counselors, and nurse practitioners, who play important roles in delivering in-person mental health services to patients with MHCs.

The role of $\mathrm{TMH}$ in delivering $\mathrm{MH}$ services varied by the type of service. $\mathrm{TMH}$ has mostly been used for outpatient/inpatient consultations (94.7\%) and pharmacological management $(53.8 \%)$. The extensive use of TMH for providing consultations may be due to the shortage of psychiatrists in the US. ${ }^{15}$ Consultations via TMH can make psychiatry services available to patients with MHCs, regardless of time and geographical barriers. ${ }^{115}$ 
Pharmacological management is an essential part of mental healthcare. Given the increasingly important role that pharmacists are playing in comprehensive medication management in collaborative care models, additional research is needed to explore the feasibility, acceptability, and outcomes of pharmacist-delivered, TMH based medication management. ${ }^{116}$

On the other hand, the delivery of psychotherapy using TMH was rare despite growing evidence supporting the effectiveness of psychotherapy delivered via TMH using a range of modalities. ${ }^{52,117,118}$ This might be due to a lack of clinician confidence and experience in delivering Tele-psychotherapy. ${ }^{31,117,119}$ A recent systematic review of studies comparing the therapeutic alliance of Tele vs. in-person psychotherapy suggested that guidelines and training on TMH delivery of psychotherapy could increase clinicians' comfort and confidence. ${ }^{118}$ The newly released guidelines on videoconferencing-based TMH services by the American Psychiatric Association and American Telemedicine Association may help to increase the use of TMH for providing psychotherapy in the future. ${ }^{114}$

Overall, TMH use was significantly associated with patient age, health insurance characteristics, and severity of MHC. We found that TMH use was more likely among patients with MHCs of younger age and among MA enrollees who were less than 65 years old, consistent with previously reported results from studies of Medicaid and Medicare populations. ${ }^{41,42}$ Although there is evidence that telehealth programs are well-accepted by elderly populations, ${ }^{120}$ home-based telehealth has not been widely covered by Medicare or most commercial plans, potentially limiting TMH use by older adults. Efforts that facilitate home-based TMH services, including expanding high-speed internet coverage and reimbursement coverage of Tele-home health, may help increase TMH use among elderly patients. ${ }^{121}$ 
Another interesting finding is that higher monthly out-of-pocket (OOP) expenditures were associated with higher odds of TMH use in both the pooled sample and MHC patients in MA plans. Higher OOP expenditures have been perceived as significant barriers to healthcare. ${ }^{45}$ While the insurance plans required some OOP costs (i.e., co-pay, coinsurance, deducible) for patients using $\mathrm{TMH}$ and in-person $\mathrm{MH}$ services, patients bearing higher direct healthcare costs might choose TMH to reduce their indirect healthcare costs (i.e., traveling time and costs). ${ }^{122,123}$

Our results indicated that patients with greater mental health care needs were more likely to use TMH services. TMH use was most prevalent among patients with schizophrenia. Furthermore, having any severe MHC diagnosis at baseline was associated with higher odds of TMH use. This might be because patients with severe MHCs have a critical need for pharmacological and psychosocial treatments, as well as specialist care. ${ }^{58}$ It has been well documented that geographical barriers such as longer travel distance to care are negatively associated with receiving mental health treatments. ${ }^{124}$ Limited access to care due to geographical barriers may have a greater impact on healthcare outcomes of adults with severe MHCs as compared to those with less severe conditions. ${ }^{125} \mathrm{TMH}$ can facilitate access to mental health specialist for these patients, especially those with limited or no access to mental health specialists. ${ }^{54}$ Furthermore, our finding that TMH use was more likely among patients with a history of any specialist visits at baseline indicated that TMH might help patients with MHCs continue specialist care with follow-up visits via TMH.

We found that TMH use was much higher among patients with MHCs who were enrolled in MA plans than those who were not. It is surprising that MA enrollees had higher TMH use because non-MA insurance plans do not limit TMH coverage to patients living in underserved rural areas, while MA plans do limit coverage. It is plausible that MHC patients in MA plans had 
more severe conditions (Supplemental table) and therefore perceive more need for mental health services in general than those who were not in MA plans.

It is worth noting that polypharmacy was associated with higher odds of TMH use among all adults with MHCs, but not among those enrolled in MA plans. Although pharmacological management was one of the most commonly used TMH services, Medicare covers this service only as part of inpatient care. Given that polypharmacy is more prevalent in the elderly population, as compared to their younger counterparts, expanding Medicare TMH coverage for pharmacological management in outpatient settings may increase TMH use by this population.

TMH use varied significantly across states despite similar TMH coverage and reimbursement policies. Results from multilevel analyses revealed that this inter-state variation could be partially explained by state-level shortages of mental health providers and differences in TMH capacity among mental health facilities. Patients from states with greater shortages or higher TMH capacity were significantly more likely to use TMH, than those without such an environment. These findings suggest that increasing TMH capacity in health facilities is an essential step in encouraging TMH use among patients with MHCs.

\section{Limitations}

The study has several strengths, including our large sample size and the identification of factors contributing to state-level variation in TMH use. We derived study cohorts from claim data of a geographically diverse population from all 50 states and DC, which supports the examination of state-level variation in $\mathrm{TMH}$ use across the nation. ${ }^{108}$ Furthermore, we used linked, nation-wide data from three databases, permitting us to capture a wide array of information regarding both patient- and state-level factors. However, several limitations should also be noted. First, we only had access to claims till June $30^{\text {th }}, 2017$, potentially leading to 
underestimation of TMH usage this year. Second, MA only covers TMH for those in underserved rural areas. Due to the lack of information on personal residency such as zip codes, the percentage of TMH users among MA enrollees with MHCs in our study may underrepresent the utilization of TMH in this population. Third, the results may be affected by unmeasured factors (e.g., race/ethnicity, education levels, patient and provider preferences) that were not captured in our data. Furthermore, as claims data do not include clinical information on disease severity (e.g., symptoms, functioning status), the number of patients with severe MHCs may have been underestimated due to our reliance on diagnostic codes. Another limitation is that subgroup analyses of TMH use among patients with a specific MHC, or among those who were only privately insured was not possible due to limited sample sizes of users in these groups. Finally, although we examined TMH use among enrollees of one of the largest commercial plans in the country, our results may not be generalizable to MHC patients insured by other commercial plans.

\subsection{Conclusion}

In summary, TMH use among adults with MHCs was low in this study of a large, commercially insured population. Psychotherapy delivered via TMH was rare relative to those delivered in-person. Substantial state-level variation in TMH use was observed even in the presence of similar reimbursement and coverage policies. Our results suggest that current TMH use among MHC patients were driven mainly by a greater need to access mental health specialists. TMH use was also particularly low among certain patient subgroups, suggesting that targeted efforts are needed to encourage TMH use in these populations. Collaborative efforts by payers and providers, particularly those that could expand TMH capacity in healthcare settings, 
could increase the availability of TMH in mental health care delivery, and thus, increase access to mental healthcare services for patients with MHCs. 
Table 3.1 Description of adults with mental health conditions (MHC) and percentages of Telemental health (TMH) users among adults with MHC.

\begin{tabular}{lccc}
\hline & \multicolumn{2}{c}{ Adults with MHCs } & TMH users\% \\
\hline & $\mathbf{N}$ & Column \% & $\mathbf{N}(\%)$ \\
All adults with MHC & 248,060 & 100.00 & $385(0.16)$ \\
Any serious MHC & 79,861 & 32.19 & $295(0.37)$ \\
Schizophrenia & 3,639 & 1.47 & $59(1.62)$ \\
Bipolar & 19,010 & 7.66 & $104(0.55)$ \\
Major depressive disorders & 61,571 & 24.82 & $178(0.29)$ \\
Any anxiety disorders & 105,501 & 42.53 & $200(0.19)$ \\
Year of TMH use & & & \\
2010 & 43,256 & 17.44 & $11(0.03)$ \\
2011 & 36,680 & 14.79 & $24(0.07)$ \\
2012 & 34,780 & 14.02 & $25(0.07)$ \\
2013 & 31,761 & 12.80 & $41(0.13)$ \\
2014 & 31,847 & 12.84 & $49(0.15)$ \\
2015 & 34,111 & 13.75 & $80(0.24)$ \\
2016 & 31,827 & 12.83 & $129(0.41)$ \\
2017 & 3,798 & 1.53 & $26(0.69)$ \\
\hline
\end{tabular}

Patient-level: Biological factors ${ }^{\dagger}$

Age [mean (standard deviation)], years $49.2(18.4)$

$58.1(15.8)$

Sex
Female
152,498
61.49
$233(0.15)$

Male

95,524

38.51

$152(0.16)$

Patient-level: Health insurance characteristics ${ }^{\dagger}$

Type of insurance

Medicare Advantage (MA)

Non-MA

$\begin{array}{ccc}70,466 & 28.41 & 325(0.46) \\ 177,594 & 71.59 & 60(0.03)\end{array}$

Plan type

Health Maintenance Organization (HMO)

$54,799 \quad 22.09$

$174(0.32)$

Non-HMO

$193,261 \quad 77.91$

$211(0.11)$

Monthly out-of-pocket expenditures

Zero

$146,379 \quad 59.01$

$131(0.09)$

Low ( $1^{\text {st }} \& 2^{\text {nd }}$ Quartile)

$39,673 \quad 15.99$

$104(0.26)$

High $\left(3^{\text {rd }} \& 4^{\text {th }}\right.$ Quartile $)$

$62,008 \quad 25.00$

$150(0.24)$

Patient-level: Need factors ${ }^{\dagger}$

\section{Any severe MHC *}

Yes

80,860

32.60

$295(0.37)$

No

167,200

67.40

$90(0.05)$

Any mental health specialist visit

Yes

$54,397 \quad 21.93$

$280(0.52)$

No

193,663

78.07

$105(0.05)$

Number of physical chronic conditions ${ }^{\S}$

\begin{tabular}{lccc} 
None & 117,389 & 47.32 & $76(0.07)$ \\
$1-2$ & 49,445 & 19.93 & $72(0.15)$ \\
\hline
\end{tabular}




\begin{tabular}{|c|c|c|c|}
\hline 3 or more & 81,226 & 32.74 & $237(0.29)$ \\
\hline \multicolumn{4}{|l|}{ Polypharmacy ${ }^{\mathbb{I I}}$} \\
\hline Yes & 69,039 & 27.83 & $210(0.30)$ \\
\hline No & 179,021 & 72.17 & $175(0.10)$ \\
\hline \multicolumn{4}{|c|}{ Any tobacco/alcohol use disorder } \\
\hline Yes & 41,804 & 16.85 & $86(0.21)$ \\
\hline No & 206,256 & 83.15 & $299(0.15)$ \\
\hline \multicolumn{4}{|l|}{ Any drug use disorder } \\
\hline Yes & 14,435 & 5.82 & $71(0.29)$ \\
\hline No & 233,625 & 94.18 & $314(0.14)$ \\
\hline \multicolumn{4}{|l|}{ Obesity } \\
\hline Yes & 20,251 & 8.16 & $65(0.32)$ \\
\hline No & 227,809 & 91.84 & $320(0.14)$ \\
\hline \multicolumn{4}{|c|}{ State-level: Environmental factors } \\
\hline \multicolumn{4}{|l|}{ Poverty $^{\dagger \dagger}$} \\
\hline Low ( $1^{\text {st }} \& 2^{\text {nd }}$ Quartile $)$ & 151,530 & 61.09 & $207(0.14)$ \\
\hline High $\left(3^{\text {rd }} \& 4^{\text {th }}\right.$ Quartile $)$ & 96,530 & 38.91 & $178(0.18)$ \\
\hline \multicolumn{4}{|l|}{ Rurality ${ }^{\dagger \dagger}$} \\
\hline Low ( $1^{\text {st }} \& 2^{\text {nd }}$ Quartile $)$ & 103,685 & 41.80 & $125(0.09)$ \\
\hline High $\left(3^{\text {rd }} \& 4^{\text {th }}\right.$ Quartile $)$ & 144,375 & 58.20 & $260(0.25)$ \\
\hline \multicolumn{4}{|c|}{ Mental health provider shortage ${ }^{\dagger \dagger}$} \\
\hline Low ( $1^{\text {st }} \& 2^{\text {nd }}$ Quartile $)$ & 112,535 & 45.37 & $115(0.09)$ \\
\hline High $\left(3^{\text {rd }} \& 4^{\text {th }}\right.$ Quartile $)$ & 135,525 & 54.63 & $270(0.24)$ \\
\hline \multicolumn{4}{|l|}{ TMH capacity $\#$} \\
\hline $1^{\text {st }}$ Quartile & 64,948 & 26.18 & $24(0.04)$ \\
\hline $2^{\text {nd }}$ Quartile & 75,345 & 30.37 & $99(0.13)$ \\
\hline $3^{\text {rd }}$ Quartile & 50,319 & 20.29 & $155(0.31)$ \\
\hline $4^{\text {th }}$ Quartile & 57,448 & 23.16 & $107(0.19)$ \\
\hline
\end{tabular}

Note: ${ }^{\dagger}$ all patient-level factors were measured in the six-month period before index dates based on deidentified claims of Optum Clinformatics ${ }^{\circledR}$ DataMart. The first TMH use date was used as index date for users and a pseudo-index date was randomly selected from all mental health service dates among non-users.

* the severity of mental health conditions was measured with a algorithm developed by researchers from the Healthcare Cost and Utilization Project based on diagnostic codes from claims.

$\S$ chronic conditions examined included arthritis, asthma, coronary artery disease, cardiac arrhythmias, congestive heart failure, chronic kidney disease, chronic obstructive pulmonary disease, dementia and related disorders, diabetes, hypertension, hyperlipidemia, osteoporosis, stroke.

II polypharmacy was defined as having more than five medications from different therapeutic classes in the 90 days before index date.

${ }^{\dagger}$ poverty, rurality, and mental health provider shortage were measured as average percentages of counties under qualified for each condition from 2010-2016 based on data from the 2016-2017 Area Health Resources File.

$\#$ TMH capacity was measured as average percentages of mental health facilities with telemedicine from 2010-2016 based on data from the 2010-2016 National Mental Health Services Survey. 
Table 3.2 Results of multilevel logistic regressions on Telemental health use (yes/no) among adults with mental health conditions (MHCs).

\begin{tabular}{|c|c|c|c|c|}
\hline & Model 1 & Model 2 & Model 3 & Model 4 \\
\hline & AOR $(95 \% \mathrm{CI})$ & AOR $(95 \% \mathrm{CI})$ & AOR $(95 \% \mathrm{CI})$ & AOR $(95 \% \mathrm{CI})$ \\
\hline Year of TMH use & $1.45[1.37,1.53]^{\text {*** }}$ & $1.45[1.37,1.53]^{* * *}$ & $1.45[1.37,1.53]^{* * *}$ & $1.44[1.36,1.53]^{* * *}$ \\
\hline \multicolumn{5}{|c|}{ Patient-level: Biological factors ${ }^{\dagger}$} \\
\hline Age (years) & $0.98[0.97,0.99]^{* * *}$ & $0.98[0.97,0.99]^{* * *}$ & $0.98[0.97,0.99]^{* * *}$ & $0.98[0.97,0.99]^{* *}$ \\
\hline \multicolumn{5}{|l|}{ Sex } \\
\hline Female & $1.16[0.93,1.44]$ & $1.16[0.94,1.44]$ & $1.16[0.94,1.43]$ & $1.16[0.94,1.44]$ \\
\hline \multicolumn{5}{|l|}{ Male } \\
\hline \multicolumn{5}{|c|}{ Patient-level: Health insurance characteristics ${ }^{\dagger}$} \\
\hline \multicolumn{5}{|l|}{ Type of plan } \\
\hline MA & $\begin{array}{c}15.70 \\
{[10.66,23.11]^{* * *}}\end{array}$ & $\begin{array}{c}15.72 \\
{[10.68,23.14]^{* * *}}\end{array}$ & $\begin{array}{c}15.92 \\
{[10.81,23.45]^{* * *}}\end{array}$ & $\begin{array}{c}15.94 \\
{[10.83,23.47]^{* * *}}\end{array}$ \\
\hline \multicolumn{5}{|l|}{ Non-MA } \\
\hline \multicolumn{5}{|l|}{ Plan type } \\
\hline HMO & $1.25[0.97,1.62]$ & $1.25[0.96,1.61]$ & $1.25[0.96,1.61]$ & $1.27[0.98,1.65]$ \\
\hline \multicolumn{5}{|l|}{ Non-HMO } \\
\hline \multicolumn{5}{|c|}{ Monthly out-of-pocket expenditures } \\
\hline Low & $1.48[1.13,1.95]^{* *}$ & $1.48[1.12,1.94]^{* *}$ & $1.48[1.13,1.95]^{* *}$ & $1.48[1.13,1.95]^{* *}$ \\
\hline High & $1.89[1.45,2.45]^{* * *}$ & $1.88[1.45,2.45]^{* * *}$ & $1.89[1.45,2.45]^{* * *}$ & $1.89[1.45,2.46]^{* * *}$ \\
\hline Zero & & & & \\
\hline
\end{tabular}

\section{Patient-level: Need factors}

\begin{tabular}{|c|c|c|c|c|}
\hline \multicolumn{5}{|c|}{ Any severe $\mathrm{MHC}^{\S}$} \\
\hline Yes & $\begin{array}{c}2.14 \\
{[1.66,2.76]^{* * *}}\end{array}$ & $\begin{array}{c}2.14 \\
{[1.66,2.76]^{* * *}}\end{array}$ & $\begin{array}{c}2.14 \\
{[1.66,2.76]^{* * * *}}\end{array}$ & $\begin{array}{c}2.14 \\
{[1.66,2.76]^{* * * *}}\end{array}$ \\
\hline \multicolumn{5}{|l|}{ No } \\
\hline \multicolumn{5}{|c|}{ Any in-person mental health specialist visit } \\
\hline Yes & $9.39[\underset{* * *}{7.34}, 12.03]$ & $9.41[7.35,12.04]^{* * *}$ & $9.45[7.38,12.10]^{* * *}$ & $9.46[7.39,12.11]^{* * *}$ \\
\hline \multicolumn{5}{|l|}{ No } \\
\hline \multicolumn{5}{|c|}{ Number of physical chronic conditions II } \\
\hline $1-2$ & $1.32[0.93,1.87]$ & $1.32[0.93,1.87]$ & $1.33[0.93,1.87]$ & $1.32[0.93,1.87]$ \\
\hline 3 or more & $1.35[0.97,1.87]$ & $1.35[0.97,1.88]$ & $1.35[0.97,1.88]$ & $1.35[0.97,1.88]$ \\
\hline \multicolumn{5}{|c|}{ None } \\
\hline \multicolumn{5}{|c|}{ Polypharmacy ${ }^{\dagger+}$} \\
\hline Yes & $1.41[1.09,1.82]^{* *}$ & $1.42[1.10,1.83]^{* *}$ & $1.42[1.10,1.84]^{* *}$ & $1.42[1.10,1.83]^{* *}$ \\
\hline \multicolumn{5}{|l|}{ No } \\
\hline \multicolumn{5}{|c|}{ Any tobacco/alcohol use disorder } \\
\hline Yes & $1.03[0.79,1.34]$ & $1.03[0.79,1.34]$ & $1.03[0.79,1.34]$ & $1.03[0.79,1.34]$ \\
\hline \multicolumn{5}{|l|}{ No } \\
\hline \multicolumn{5}{|c|}{ Any drug use disorder } \\
\hline Yes & $1.23[0.89,1.70]$ & $1.23[0.89,1.70]$ & $1.23[0.89,1.70]$ & $1.23[0.89,1.70]$ \\
\hline \multicolumn{5}{|l|}{ No } \\
\hline Obesity & & & & \\
\hline
\end{tabular}




\begin{tabular}{|c|c|c|c|c|}
\hline $\begin{array}{l}\text { Yes } \\
\text { No }\end{array}$ & $1.08[0.81,1.44]$ & $1.08[0.80,1.44]$ & $1.08[0.80,1.44]$ & $1.08[0.80,1.44]$ \\
\hline \multicolumn{5}{|c|}{ State level: Environmental factors } \\
\hline Poverty & - & & & \\
\hline $\begin{array}{l}\text { High } \\
\text { Low }\end{array}$ & & $0.87[0.32,2.34]$ & $0.68[0.27,1.68]$ & $0.62[0.27,1.43]$ \\
\hline \multicolumn{5}{|l|}{ Rurality $*$} \\
\hline $\begin{array}{l}\text { High } \\
\text { Low }\end{array}$ & - & $2.43[0.90,6.54]$ & $1.03[0.36,2.91]$ & $0.94[0.35,2.47]$ \\
\hline \multicolumn{5}{|c|}{ Mental health provider shortage } \\
\hline $\begin{array}{l}\text { High } \\
\text { Low }\end{array}$ & - & - & $5.38[1.87,15.50]^{* *}$ & $4.65[1.72,12.60]^{* *}$ \\
\hline \multicolumn{5}{|l|}{ TMH capacity $\$$} \\
\hline $2^{\text {nd }}$ quartile & - & - & - & $2.62[1.09,6.28]^{*}$ \\
\hline $3^{\text {rd }}$ quartile & - & - & - & $2.53[0.98,6.55]$ \\
\hline $4^{\text {th }}$ quartile & - & - & - & $1.65[0.55,4.95]$ \\
\hline $1^{\text {st }}$ quartile & & & & \\
\hline \multicolumn{5}{|c|}{ Random-effect parameters } \\
\hline LR test IIII & $503.60^{* * *}$ & $451.45^{* * *}$ & $315.86^{* * *}$ & $186.67^{* * * *}$ \\
\hline Residual variance & $2.51(0.75)$ & $2.28(0.68)$ & $1.74(0.54)$ & $1.38(0.47)$ \\
\hline VPC ${ }^{\dagger \dagger}$ & 0.43 & 0.41 & 0.35 & 0.30 \\
\hline
\end{tabular}

Note: AOR (95\%CI): adjusted odds ratio and associated $95 \%$ confidence interval estimated from multilevel logistic regressions.

HMO: health maintenance organization. Non-HMO include plans from preferred provider organizations, point of services, fee-for-service, and others.

MA: Medicare Advantage. Non-MA plans were other commercial insurances.

${ }^{\dagger}$ all patient-level factors were measured in the six-month period before index dates based on de-identified claims of Optum Clinformatics ${ }^{\circledR}$ DataMart. The first TMH use date was used as index date for users and a pseudo-index date was randomly selected from all mental health service dates among non-users.

$¥$ average monthly out-of-pocket (OOP) expenditures were categorized as zero, low, and high. Low/high OOP expenditures were defined as the lower and upper $50^{\text {th }}$ percentiles.

$\S$ the severity of mental health conditions was measured with a algorithm developed by researchers from the Healthcare Cost and Utilization Project based on diagnostic codes from claims.

"I chronic conditions examined included arthritis, asthma, coronary artery disease, cardiac arrhythmias, congestive heart failure, chronic kidney disease, chronic obstructive pulmonary disease, dementia and related disorders, diabetes, hypertension, hyperlipidemia, osteoporosis, stroke.

${ }^{\dagger \dagger}$ polypharmacy was defined as having more than five medications from different therapeutic classes in the 90 days before index date.

\# poverty, rurality, and mental health provider shortage were measured as average percentages of counties under qualified for each condition from 2010-2016 based on data from the 2016-2017 Area Health Resources File. Low/high levels were defined as the lower and upper $50^{\text {th }}$ percentiles.

$\$$ TMH capacity was measured as average percentages of mental health facilities with telemedicine from 20102016 based on data from the 2010-2016 National Mental Health Services Survey.

III Log-likelihood test was used to assess the significance of multilevel models against single-level models.

It† VPCs were calculated with the formula: level-2 residual variance / (level-1 residual variance + level-2 residual variance), where level-1 residual variance for logistic model $=3.29$.

${ }^{*}, \mathrm{p}<0.05 ;{ }^{* *}, \mathrm{p}<0.01 ;{ }^{* * *}, \mathrm{p}<0.001$. 
Table 3.3 Results of multilevel logistic regressions on Telemental health use (yes/no) among Medicare Advantage enrollees with mental health conditions (MHC) who lived in underserved states.

\begin{tabular}{|c|c|}
\hline & AOR (95\% CI) \\
\hline Year of TMH use & $1.37[1.28,1.47]^{* * * *}$ \\
\hline \multicolumn{2}{|c|}{ Patient-level: Predisposing factors ${ }^{\dagger}$} \\
\hline \multicolumn{2}{|c|}{ 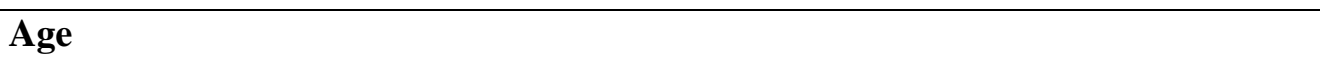 } \\
\hline $18-64$ years & $1.99[1.52,2.60]^{* * *}$ \\
\hline 65 years or older & \\
\hline \multicolumn{2}{|l|}{ Sex } \\
\hline Female & $1.19[0.93,1.52]$ \\
\hline Male & \\
\hline \multicolumn{2}{|c|}{ Patient-level: Health insurance characteristics ${ }^{\dagger}$} \\
\hline \multicolumn{2}{|l|}{ Plan type } \\
\hline Health Maintenance Organization (HMO) & $0.84[0.62,1.13]$ \\
\hline Non-HMO & \\
\hline \multicolumn{2}{|l|}{ Monthly out-of-pocket expenditures } \\
\hline Low $\left(1^{\text {st }} \& 2^{\text {nd }}\right.$ quartile $)$ & $1.39[1.02,1.89]^{*}$ \\
\hline High $\left(3^{\text {rd }} \& 4^{\text {th }}\right.$ quartile $)$ & $1.57[1.15,2.13]^{* *}$ \\
\hline Zero & \\
\hline \multicolumn{2}{|c|}{ Patient-level: Need factors ${ }^{\dagger}$} \\
\hline \multicolumn{2}{|l|}{ Any severe MHC } \\
\hline Yes & $1.83[1.36,2.46]^{* * *}$ \\
\hline No & \\
\hline \multicolumn{2}{|l|}{ Any in-person mental health specialist visit } \\
\hline Yes & $9.86[7.40,13.14]^{* * *}$ \\
\hline No & \\
\hline \multicolumn{2}{|l|}{ Number of physical chronic conditions } \\
\hline $1-2$ & $1.24[0.81,1.90]$ \\
\hline 3 or more & $1.25[0.86,1.82]$ \\
\hline None & \\
\hline \multicolumn{2}{|l|}{ Polypharmacy ${ }^{\S}$} \\
\hline Yes & $1.19[0.90,1.58]$ \\
\hline No & \\
\hline \multicolumn{2}{|l|}{ Any tobacco/alcohol use disorder } \\
\hline Yes & $0.98[0.73,1.33]$ \\
\hline No & \\
\hline \multicolumn{2}{|l|}{ Any drug use disorder } \\
\hline Yes & $1.18[0.81,1.72]$ \\
\hline No & \\
\hline \multicolumn{2}{|l|}{ Obesity } \\
\hline Yes & $1.01[0.73,1.41]$ \\
\hline No & \\
\hline
\end{tabular}




\section{State level: Environmental factors}

\begin{tabular}{|c|c|c|}
\hline \multicolumn{3}{|l|}{ Poverty ${ }^{\text {II }}$} \\
\hline & $\operatorname{High}\left(3^{\text {rd }} \& 4^{\text {th }}\right.$ quartile $)$ & $0.50[0.24,1.05]$ \\
\hline & Low $\left(1^{\text {st }} \& 2^{\text {nd }}\right.$ quartile $)$ & \\
\hline \multicolumn{3}{|l|}{ Rurality ${ }^{\text {II }}$} \\
\hline & $\operatorname{High}\left(3^{\text {rd }} \& 4^{\text {th }}\right.$ quartile $)$ & $0.80[0.34,1.84]$ \\
\hline & Low ( $1^{\text {st }} \& 2^{\text {nd }}$ quartile $)$ & \\
\hline \multicolumn{3}{|c|}{ Mental health provider shortage II } \\
\hline & High $\left(3^{\text {rd }} \& 4^{\text {th }}\right.$ quartile $)$ & $4.29[1.79,10.29]^{* *}$ \\
\hline & Low $\left(1^{\text {st }} \& 2^{\text {nd }}\right.$ quartile $)$ & \\
\hline \multicolumn{3}{|c|}{ TMH capacity ${ }^{\dagger \dagger}$} \\
\hline & High $\left(3^{\text {rd }} \& 4^{\text {th }}\right.$ quartile $)$ & $1.53[0.88,2.65]$ \\
\hline & Low $\left(1^{\text {st }} \& 2^{\text {nd }}\right.$ quartile $)$ & \\
\hline \multicolumn{3}{|c|}{ Random-effect parameters } \\
\hline & Likelihood Ratio test vs. logistic model & $76.94^{* * *}$ \\
\hline & Residual variance & $1.00(0.38)$ \\
\hline & Variance Partition Coefficients (VPCs) & 0.23 \\
\hline
\end{tabular}

Note: AOR (95\%CI): adjusted odds ratio and associated 95\% confidence interval estimated from multilevel logistic regressions. Underserved states were defined as states with high percentages of rural counties or counties with shortage of healthcare providers. ${ }^{\dagger}$ all patient-level factors were measured in the six-month period before first TMH use based on de-identified claims of Optum Clinformatics ${ }^{\circledR}$ DataMart. The first TMH use date was used as index date for users and a pseudo-index date was randomly selected from all mental health service dates among non-users.

$\$$ chronic conditions examined included arthritis, asthma, coronary artery disease, cardiac arrhythmias, congestive heart failure, chronic kidney disease, chronic obstructive pulmonary disease, dementia and related disorders, diabetes, hypertension, hyperlipidemia, osteoporosis, stroke.

${ }^{\S}$ polypharmacy was defined as having more than five medications from different therapeutic classes in the 90 days before index date.

II poverty, rurality, and mental health provider shortage were measured as average percentages of counties under qualified for each condition from 2010-2016 based on data from the 2016-2017 Area Health Resources File.

${ }^{\dagger} \mathrm{TMH}$ capacity was measured as average percentages of mental health facilities with telemedicine from 2010-2016 based on data from the 2010-2016 National Mental Health Services Survey.

H VPCs were calculated with the formula: level-2 residual variance / (level-1 residual variance + level-2 residual variance), where level-1 residual variance for logistic model $=$ 3.29 .

${ }^{*}, \mathrm{p}<0.05 ;^{* *}, \mathrm{p}<0.01 ;{ }^{* * *}, \mathrm{p}<0.001$. 


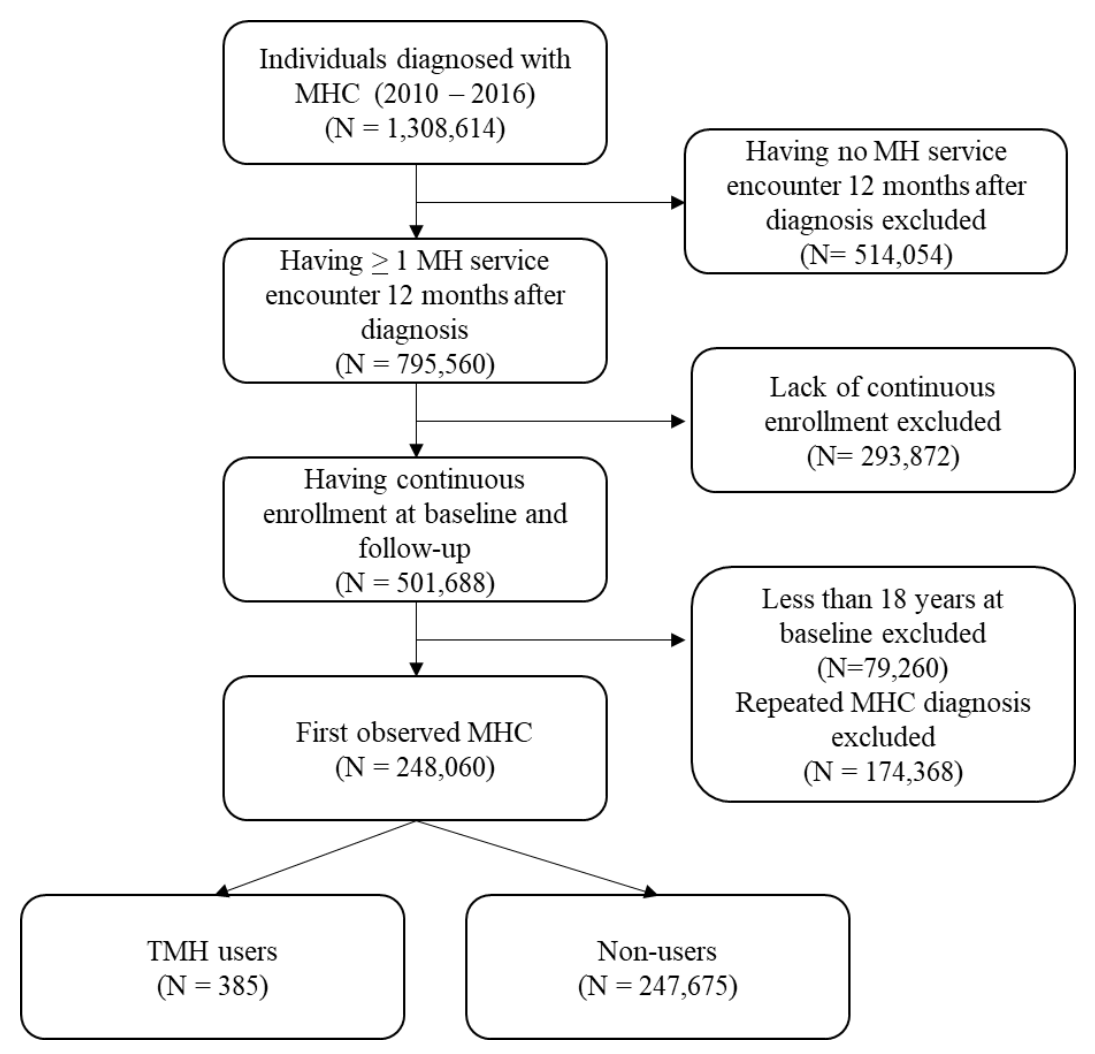

Figure 3.1 Aim 2 Cohort identification process. 


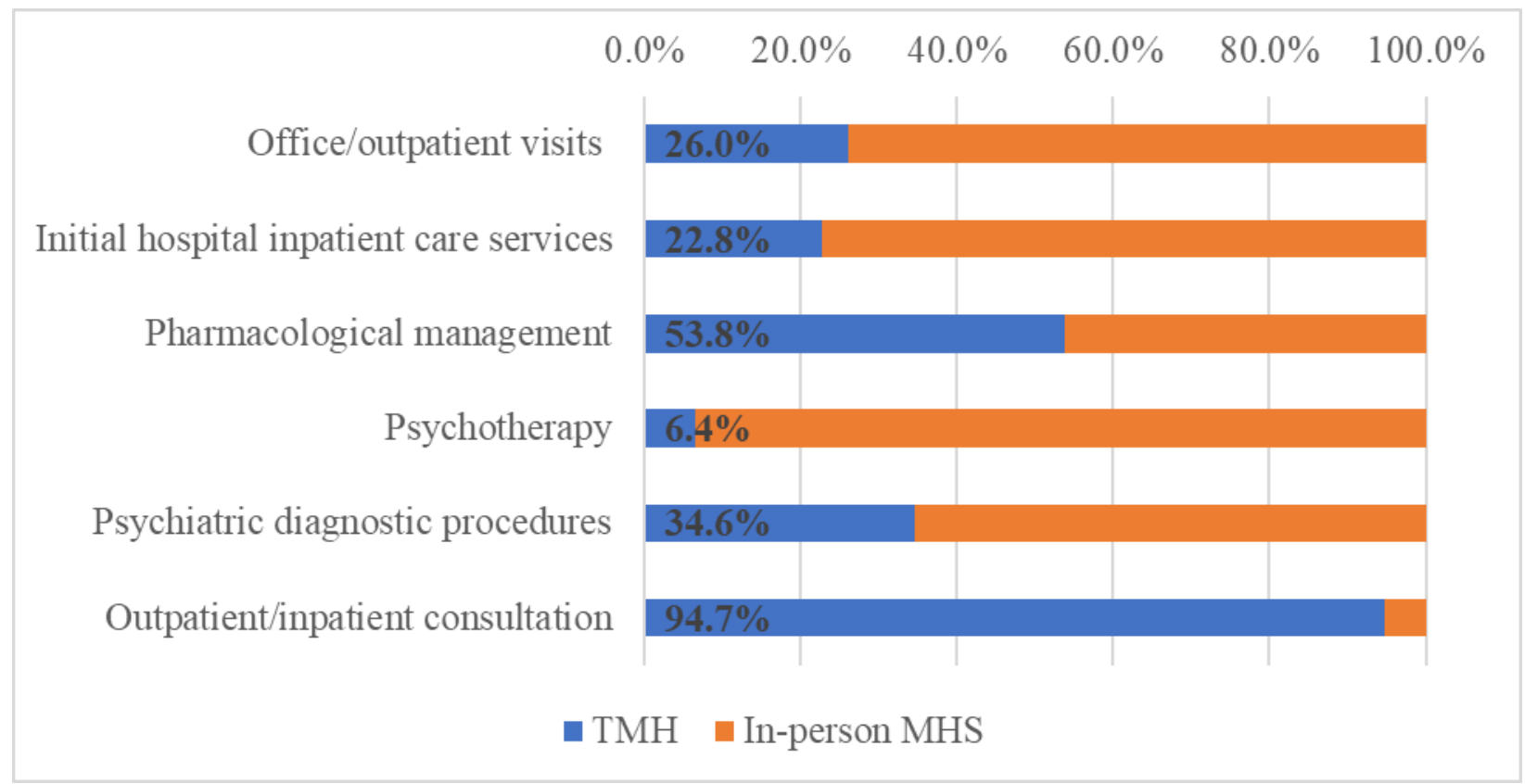

Figure 3.2 Distribution of selected mental health (MH) services delivered via Telemental health (TMH)/in-person. 


\section{CHAPTER 4}

\section{The Impact of Telemental Health (TMH) Use on Healthcare Costs Among Commercially Insured Adults with Mental Health Conditions}

\subsection{Abstract}

Objective: To determine the impact of TMH use on total healthcare costs and mental health (MH)-related costs paid by a third party among adults with mental health conditions (MHC). Method: This study employed a pre-post design with a non-equivalent control group. The cohort comprised adults with MHCs identified using diagnosis codes from de-identified claims data of the Optum Clinformatics ${ }^{\circledR}$ DataMart (01/01/2010 - 06/30/2017). We identified MH service users and TMH users $(\mathrm{N}=348)$ based on procedure codes. Non-users $(\mathrm{N}=238,595)$ were defined as those who only used in-person MH services. A Difference-in-Differences (DID) analysis was performed within a multivariable two-part model (TPM) framework to examine the impact of TMH use on adjusted standardized costs (2018 US \$) of all healthcare services and MH services. Patient-level and state-level factors were adjusted in TPM. Results: TMH use was associated with significantly higher MH-related costs [Marginal effect $=\$ 461.3,95 \%$ confidence interval: $\$ 142.4$ - \$780.2] and an excess of $\$ 370$ increase in MH-related costs at follow-up as compared to baseline. However, TMH use was not associated with an increase in total third-party healthcare costs nor with changes in total costs from baseline to follow-up. Conclusions: Despite having a higher likelihood of MH services use and MH-related costs, TMH users did not have higher total costs as compared to adults using only in-person $\mathrm{MH}$ services. Our findings suggest that TMH can increase access to MH care without increasing total healthcare costs among adults with MHC.

\subsection{Highlights}


- Existing clinical trials have demonstrated that MH services delivered via TMH are comparable to those delivered in-person for assessing and treating various MHCs. However, there is a concern that TMH may increase healthcare utilization and costs.

- This study is the first to examine the impact of TMH use on third-party healthcare costs and mental health $(\mathrm{MH})$-related costs by comparing the changes in costs between TMH users and in-person MH service users (i.e., without TMH use) among adults with MHCs seeking care in real-world clinical settings.

- The study results suggested that TMH use was associated with an excess of $\$ 370$ in $\mathrm{MH}$ related costs from baseline to follow-up. However, TMH users did not differ significantly from non-users in average total healthcare costs. Future studies are warranted to determine if this apparent cost-offset is due to improvements in $\mathrm{MH}$ and physical health outcomes or to other factors.

\subsection{Introduction}

Mental health conditions (MHCs) are the most expensive conditions among ten conditions with fast spending growth (i.e., heart conditions, trauma, cancer) in the United States, with an estimated excess cost of $\$ 38$ billion in direct healthcare expenses in 2013. ${ }^{102}$ The high MHC prevalence coupled with continued undertreatment of MHC have contributed significantly to this high economic burden. ${ }^{8,80,103}$ Telemental health (TMH) has emerged as an alternative care delivery approach to meet the growing demand of $\mathrm{MH}$ care and shortages of $\mathrm{MH}$ providers. ${ }^{126} \mathrm{TMH}$ is the use of remote technologies such as video-conferencing systems to deliver MH care. ${ }^{106}$ Clinical trials have shown that TMH is well accepted ${ }^{107,127,128}$ and has comparable efficacy in the evaluation and treatment of MHC patients across a broad range of demographic groups. ${ }^{51-54,129}$ 
Although the comparative efficacy of TMH in delivering MH care relative to in-person care is well-documented in clinical trials,${ }^{51-54,129}$ studies on outcomes of TMH use among adults with MHCs in real-world settings are sparse, limited primarily to those using data from the Veterans Health Administration. For instance, a study examining outcomes of 98,609 veterans receiving TMH services reported a $25 \%$ reduction in psychiatric admissions and hospital stays after initiating TMH over four years. ${ }^{60}$ However, the study did not compare the changes in healthcare utilization and costs between individuals with TMH use and those with only in-person MH services use ${ }^{60}$ limiting interpretation of findings.

To establish the true value of TMH services, studies comparing healthcare utilization and costs among MHC patients receiving TMH and in-person MH services are warranted. In response to the rising healthcare costs, the Institute of Health Improvement developed the "Triple Aim" to define high-value care as care that can improve population health and individual care experience with reduced per-capita costs. ${ }^{61}$ In alignment with the "Triple Aim," the National Quality Forum (NQF) and the American Telemedicine Association use healthcare costs as one of the key measurements in their newly developed telehealth value framework. ${ }^{62}$ However, previous studies have primarily focused on indirect medical costs such as time and expenses related to travel. ${ }^{130-132}$ No study has compared the direct healthcare costs of individuals with MHC who used TMH services with that of individuals with MHC who used only in-person MH services. Understanding the relative utilization and direct care costs will help inform and guide the decisions of healthcare payers regarding the expansion of TMH coverage. ${ }^{67}$

Therefore, the primary objective of this study was to determine the impact of TMH use on total healthcare costs and mental health $(\mathrm{MH})$-related costs among adults with mental health conditions (MHC) using data from a large commercial insurer. 


\subsection{Methods}

\section{Data source}

Data (January 2010 through June 2017) were derived from Optum Clinformatics ${ }^{\circledR}$ DataMart (Eden Prairie, MN, USA), a large commercial health plan in the US Data included deidentified medical claims of physician, hospital, and prescription drug services as well as enrollment information for covered individuals from all 50 states and the District of Columbia. ${ }^{108}$ We linked claims data with that from the 2016-2017 Area Health Resources File (AHRF) ${ }^{109}$ and the 2010-2016 National Mental Health Services Survey (N-MHSS) ${ }^{78}$ using state identifiers. The linkage was conducted to obtain state-level information on poverty, rurality, and $\mathrm{MH}$ provider shortage from the AHRF, as well as the TMH capacity from N-MHSS files.

\section{Study design}

We used a pre-post study design with a non-equivalent control group. The pre- (baseline) and post- (follow up) periods each consisted of six months. For TMH users, the first TMH service date after 12 months of MHC diagnosis was used as the index date. For those who used only in-person MH services (i.e., non-users), a pseudo-index date was randomly selected from all MH service dates between 2010 and 2016 to allow a six-month follow-up. Standardized costs were assessed for both six months before (i.e., baseline) and six months after (i.e., follow-up) the index/pseudo-index dates.

\section{Study cohort}

The study cohort comprised adults ( $\geq 18$ years old at baseline) who were diagnosed with any MHC between 2010 and 2016, who had at least one MH service encounter 12months after the MHC diagnosis, and who were continuously enrolled in any health plan during the baseline and follow-up periods. We identified adults with MHC based on the primary and secondary 
diagnosis codes [the International Classification of Diseases, Ninth Revision (ICD-9) codes: 291292; 295-316; ICD-10: F10-F99] in inpatient and outpatient medical claims. ${ }^{42}$ Adults with MCH were defined as those with an $\mathrm{MCH}$ diagnosis from at least two outpatient visits or one inpatient admission. ${ }^{42} \mathrm{MH}$ service encounters were identified with procedure codes (Appendix 7.1) or primary/secondary diagnostic codes of any MHC. ${ }^{42}$ Data on adults with MHC for each year (2010-16) were combined to form the study cohort. The cohort identification process is illustrated in Figure 4.1. The final analytic cohort included 238,943 unique adults with MHC.

\section{Measures}

\section{Outcomes}

Standardized total healthcare costs included total insurer-allowed payments for inpatient stays, facility outpatient visits, professional services, and prescription drugs during the period assessed. To account for differences in allowed payments across health plans and provider contracts, standardized prices were used to represent allowed payments. Standardized prices for medical services and prescription drugs were estimated using algorithms developed by the Optum researchers, accounting for the type and quantity of services as well as the relative resource costs involved in providing the service. All the costs were normalized to 2018 US dollars using the standard prices and cost factors provided by Optum Clinformatics ${ }^{\circledR}$ DataMart.

$\underline{M H-\text { related costs }}$ were estimated by summing up all allowed payments from the insurer for MH services using standard prices and cost factors. MH services were identified by procedure or primary/secondary diagnostic codes as described above (study cohort part).

\section{Key independent variable: TMH use versus (vs.) No TMH use}

We defined comparison groups based on TMH use (yes/no), identified from MH service claims using the relevant Healthcare Common Procedure Coding System (HCPCS)/Current 
Procedural Terminology (CPT) codes (“0188T”, “0189T”, "G0406 - 0408”, "G0425 - 0427”, “G0459”) and/or modifiers (“GT”, "GQ”, "95”). ${ }^{42,49}$ These codes were listed on the policy document of the insurer and have been used in a previous study of TMH among Medicare beneficiaries. $^{42,49}$ The TMH user group included adults with MHC who had any record of TMH use; non-user group included those who used only in-person MH services.

\section{Other independent variables}

We selected other independent variables based on the adapted Andersen's Behavioral Model of Health Services Use. ${ }^{74}$ The model posits that individual healthcare utilization is a function of multiple factors, including 1) predisposing factors (age, sex); 2) enabling factors [TMH use (yes/no), type of insurance (Medicare Advantage/others), type of health plan (Health Maintenance Organization/others); 3) need factors [severe MHC diagnosis (yes/no), mental health specialist visits (yes/no); the number of physical chronic conditions (none/one or two/three or more); or the presence of polypharmacy (use of $>5$ drug classes in a 90-day period ${ }^{111}$ ), obesity, and/or any substance (i.e., alcohol, tobacco, drug) use disorders (SUD)]; and 4) environmental factors (the percentage of: counties under poverty, rural counties, counties with MH provider shortage, and MH facilities with $\mathrm{TMH}$ adoption by state). We also adjusted for the year of TMH use to account for the effect of time. Time from MHC diagnoses to index/pseudoindex dates (136 \pm 104 days) was also included in the multivariable analyses.

All need factors were identified from inpatient and outpatient claims using ICD9/ICD10 diagnostic codes. An algorithm developed by researchers of the Healthcare Cost and Utilization

Project was used to define the severity of MHCs using ICD9/10 diagnostic codes. ${ }^{112}$ The severity classes derived from this algorithm were strong predictors of hospitalizations after an Emergency department visit. ${ }^{112}$ The number of chronic physical conditions [range 0-13, based on a list 
developed by the Multiple Chronic Conditions (MCC) working group ${ }^{113}$ ] were also ascertained using ICD9/ICD10 codes from claims data. All patient-level factors were measured at the baseline and follow-up period. Environmental factors, measured at the state level, were ascertained using data from the AHRF.

\section{Statistical analyses}

Chi-square tests and independent t-tests were conducted to compare the characteristics of TMH users and non-users at baseline and follow-up, respectively. Standard linear regression is usually not appropriate for modeling costs due to certain common characteristics of cost data, including zero mass, non-negative values, and positive skewness. ${ }^{133}$ Therefore, we used a mixed distributions approach to model costs. Specifically, we used two-part models (TPM), in which the probability of non-zero costs (yes/no) was modeled with logistic regressions, and the magnitude of costs was modeled within Generalized Linear models (GLMs) using gamma distributions and log-link functions. ${ }^{133}$ The marginal effect (ME) and associated $95 \%$ confidence interval $(95 \% \mathrm{CI})$ were used to represent the parameter estimate. As need factors were derived from claims of service encounters, we adjusted for need factors in GLM but not in the logistic regression analyses. We adjusted for all other independent variables in both parts of TPM; these variables include the year of TMH use, time from MHC diagnosis to index/pseudo-index dates, and predisposing-, enabling-, and environmental factors. A Difference-in-Differences (DID) estimate was included in the TPMs to estimate the excess change in both total third-party healthcare costs and $\mathrm{MH}-$ related costs due to TMH use. The DID estimate is an interaction term of TMH use (yes vs. no) and time (follow-up vs. baseline), representing the effect of using TMH on cost changes from baseline to follow-up. ${ }^{134}$ For all analyses in this study, a $p$-value less than 
0.05 was considered statistically significant. All data management and analyses were performed with SAS 9.4 (SAS Institute Inc., Cary, NC) and Stata 14 (StataCorp LLC, College Station, TX).

\subsection{Results}

\section{Sample description}

A total of 238,943 adults with MHC were identified. The most prevalent MHC was anxiety disorder (42.4\%). About one-third of the study sample had serious MHCs such as schizophrenia (1.4\%), bipolar disorder (7.6\%), and major depressive disorder (24.7\%). The sample was predominantly female $(61.5 \%)$, with a mean age of 48.5 years $(\mathrm{SD}=18.3)$.

Overall, only 15 in 10,000 adults with MHCs used TMH (Table 1). At both baseline and follow-up, TMH users differed significantly from non-users in age and in all enabling-, need-, and environmental factors and non-users (Table 1). For example, at baseline, a higher percentage of TMH users had received a serious MHC diagnosis $(75.6 \%$ vs. $32.2 \%, \mathrm{p}<0.001)$ and had visited a mental health specialist $(51.2 \%$ vs. $19.2 \%, \mathrm{p}<0.001)$ as compared to non-users. Furthermore, a greater percentage of TMH users than non-users had a record of polypharmacy (54.3\% vs. $27.7 \%, \mathrm{p}<0.001)$ and had been diagnosed with at least two comorbid chronic conditions $(61.2 \%$ vs. $32.7 \%, \mathrm{p}<0.001)$. The distribution of all patient-level factors within each group was similar at baseline and follow-up (Table 1).

\section{Unadjusted and adjusted MH-related costs}

As illustrated in Table 2, "within" group differences in MH-related costs varied between TMH users and non-users. While a similar percentage of TMH users received MH services at baseline and follow-up (29.9\% vs. $33.3 \%, \mathrm{p}=0.548)$, the percentage of non-users receiving $\mathrm{MH}$ services declined significantly (from $13.9 \%$ to $8.3 \%, \mathrm{p}<0.001$ ) from the baseline to the followup period. Similarly, average MH-related costs for TMH users did not change significantly from 
baseline to follow-up $(\$ 9,703.7$ vs. $\$ 11,009.1, \mathrm{p}=0.643)$. In contrast, the average $\mathrm{MH}$-related costs for non-users increased significantly during this period (from $\$ 7,417.3$ to $\$ 8,894.3$, $\mathrm{p}<$ $0.001)$.

Between group comparisons using DID analysis indicated that TMH users were over twice as likely than non-users to utilize MH services after adjustment for other factors (AOR: 2.06, 95\% CI: $1.62-2.63)$. Based on the marginal effect estimate, TMH use was also associated with $\$ 461.3$ higher MH-related costs. Furthermore, after adjusting for other factors, TMH user had increased MH-related costs at follow-up as compared to baseline, and these increased costs were $\$ 369.6$ higher than those among non-users, after adjusting for other factors. (Table 3).

Other factors associated with higher MH-related costs included MA enrollment, all need factors such as having polypharmacy, any SUD, and multiple chronic physical conditions, and living in states with high levels of poverty (Appendix 7.2 supplemental table).

\section{Unadjusted and adjusted total healthcare costs}

Table 2 presents the average unadjusted total costs and MH-related costs among TMH users and non-users at baseline and follow-up. When comparing follow-up utilization and costs to those at baseline, TMH users had similar percentages of individuals with zero costs $(5.7 \%$ vs. $6.0 \%)$ and average total healthcare costs $(\$ 12,960.1$ vs. $\$ 13,336.6)$ at baseline and follow-up. However, among non-users, a significantly higher percentage of individuals had zero costs at follow-up than at baseline (7.9\% vs. $7.1 \%, \mathrm{p}<0.001)$, with a corresponding decrease in average total healthcare costs $(\$ 6,115.6$ at follow-up vs. $\$ 6,642.3$ at baseline, $p<0.001)$.

Group difference in differences from baseline to follow-up were examined with multivariable DID analysis within a TPM framework. Results from DID analysis indicated that TMH use $(\mathrm{ME}=-\$ 253.9,95 \% \mathrm{CI}:-\$ 1,601.5-\$ 1,093.7)$ was not associated with total healthcare 
costs. Furthermore, TMH use (ME of DID estimate $=\$ 554.3,95 \%$ CI: $-\$ 1,005.2-\$ 2,113.8)$ had no significant impact on the change in healthcare costs from baseline to follow-up (Table 3). Other factors contributing to higher total third-party costs were similar to those for higher $\mathrm{MH}$ related costs (Appendix 7.2 supplemental table).

\subsection{Discussion}

Our study is the first to compare direct total third-party healthcare costs and MH-related costs among adults with MHCs who used TMH services and those used only in-person MH services (i.e., non-users). It has been reported that payers may be less likely to adopt TMH due to the fear of higher utilization and costs associated with $\mathrm{TMH}{ }^{135}$. Our findings suggest that TMH use did not have an impact on total healthcare costs. However, TMH use was associated with a greater likelihood of using MH services, suggesting that TMH may have increased access to MH services for adults with MHC. Although our study did not have information on wait time, published studies suggest that TMH may contribute to increased utilization by reducing the wait time for MH services. For example, in a study evaluating a Telemental health clinic, the number of days to the first service was $42 \%$ shorter for services delivered via TMH than in-person services (19.1 vs. 33.1, $\mathrm{p}<0.001)$, and the wait time for medication management via TMH was $43.2 \%$ of that for in-person services ${ }^{136}$.

It is worth noting that, in our study, TMH was associated with increased MH-related costs but not total healthcare costs, suggesting that reduced use of non-MH services may offset the incremental costs from using $\mathrm{MH}$ services. Although we were not able to analyze the source of this cost-offset (e.g., reduced inpatient use, reduced emergency room visits) due to small sample sizes, it is plausible that TMH may have positive spill-over effects on physical health, resulting in decreased use of non-MH services. Such "cost-offset effect" following MH treatment 
has been reported in other clinical populations, including adults with co-existing depression and diabetes ${ }^{137}$. As a key priority of the patient-centered medical home model (PCMH) is to integrate mental and physical health, TMH may offer unique opportunities to achieve such integration ${ }^{138}$. Future study is needed to explore how to incorporate TMH in the PCMH model. The increased costs of MH services might also be offset by TMH-facilitated medication management. Polypharmacy was prevalent among adults with MHC in our study, characterizing $27.8 \%$ of adults with MHC overall and 56.6\% of those with TMH use (Table 1). We found that the presence of polypharmacy was significantly associated with both higher healthcare costs and MH-related costs (Appendix 7.2 supplemental table). Without proper management, polypharmacy has been shown to increase healthcare costs due to elevated risks of adverse drug reactions and drug-drug interactions ${ }^{139}$. Medication management is one of the most commonly used TMH services ${ }^{140}$. Studies have demonstrated the efficacy of TMH in improving medication adherence and associated clinical outcomes ${ }^{141,142}$. Furthermore, TMH allows for frequent short visits if necessary, such as those for reviewing medications, a feature that is especially valuable for patients for whom travel is difficult due to distance, weather, poor health, or other challenges (i.e., obesity).

In brief, findings of our study suggest TMH may expand access to MH services without increasing total healthcare costs. Access to SUD treatments is even more limited than access to MH services, with only one in ten individuals with SUD received treatments ${ }^{143}$. As SUD and MH treatments share common barriers (e.g., provider shortage, stigma), much attention has been focused on the potential for using telehealth to increase access to SUD care ${ }^{97,144}$. Having any substance use disorders (SUD) was a significant contributor to overall healthcare $(\$ 3,981.2$ \$4,798.9) costs and MH-related costs (\$201.3-\$382.9) in our study (Appendix 7.2 supplemental 
Table). Cost-saving might be achieved in the future by employing telehealth for delivery of SUD care. A recent study of claims data had reported a very low rate of use (0.62 visits/1,000 patients) of telehealth in treating SUD in real-world practice ${ }^{110}$. The low usage was partially due to regulatory and reimbursement barriers in prescribing SUD treatments via telehealth approach ${ }^{110}$. Given that SUD and MHC often co-occur, expanding telehealth coverage for SUD may also help reduce healthcare costs among adults with MHCs.

This study has several strengths, including the population-based design and comparison of TMH vs in person mental health care use in a real-world setting. We standardized prices of medical services and prescriptions to estimate healthcare costs to facilitate comparison across patients with different health plans and provider contracts. Our study cohort comprised a geographically diverse sample of adults with different MHCs, increasing generalizability of the study findings. Furthermore, we employed a pre-post DID study design, which allowed us to account for certain unobserved factors (e.g., disease severity) that may affect healthcare costs ${ }^{134}$.

Several limitations of the study should also be noted. First, our study estimated only direct healthcare costs and our study population was restricted to adults enrolled in a single private insurance plan. Indirect healthcare costs such as those related to travel of patients, their family members, and providers were not considered. Given that multiple studies have demonstrated that TMH can reduce indirect healthcare costs due to lost productivity (e.g., time off from work) and transportation expenses ${ }^{54,64,145}$, indirect $\mathrm{MH}$ costs are likely to be comparable or lower for MHC patients who used TMH as compared to those used in-person $\mathrm{MH}$ services. Second, we only estimated costs for MH services. Costs of prescription medications used to treat MHCs were not included because some of these medications (e.g., antidepressants) are often used to treat physical conditions such as pain and insomnia ${ }^{146}$. Third, due to the limited 
sample size, we did not conduct subgroup analyses to compare the costs of specific MH services delivered via TMH vs. in-person. Furthermore, as this study compared short-term (i.e., six months) healthcare costs between TMH users and non-users. The long-term impact of TMH use on healthcare costs among adults with MHCs thus warrants further examination. Finally, although our study cohort was drawn from enrollees in one of the largest commercial plans in the country, our results may not be generalizable to MHC patients insured by other commercial plans.

\subsection{Conclusion}

This is the first study to examine the association between TMH use and direct healthcare costs over time from a private payer's perspective. TMH use was associated with an increased utilization of MH services and a corresponding excess in MH-related costs due to. However, TMH use did significantly affect total third-party healthcare costs. Future studies should examine the sources of this apparent "cost-offset" and explore whether TMH use can lead to cost-savings over a longer period. 
Table 4.1 Characteristics of adults with mental health conditions (MHCs) who used Telemental health (TMH) and those used in-person mental health (MH) services at baseline and follow-up, Optum Clinformatics ${ }^{\circledR}$ DataMart, 2010-2017

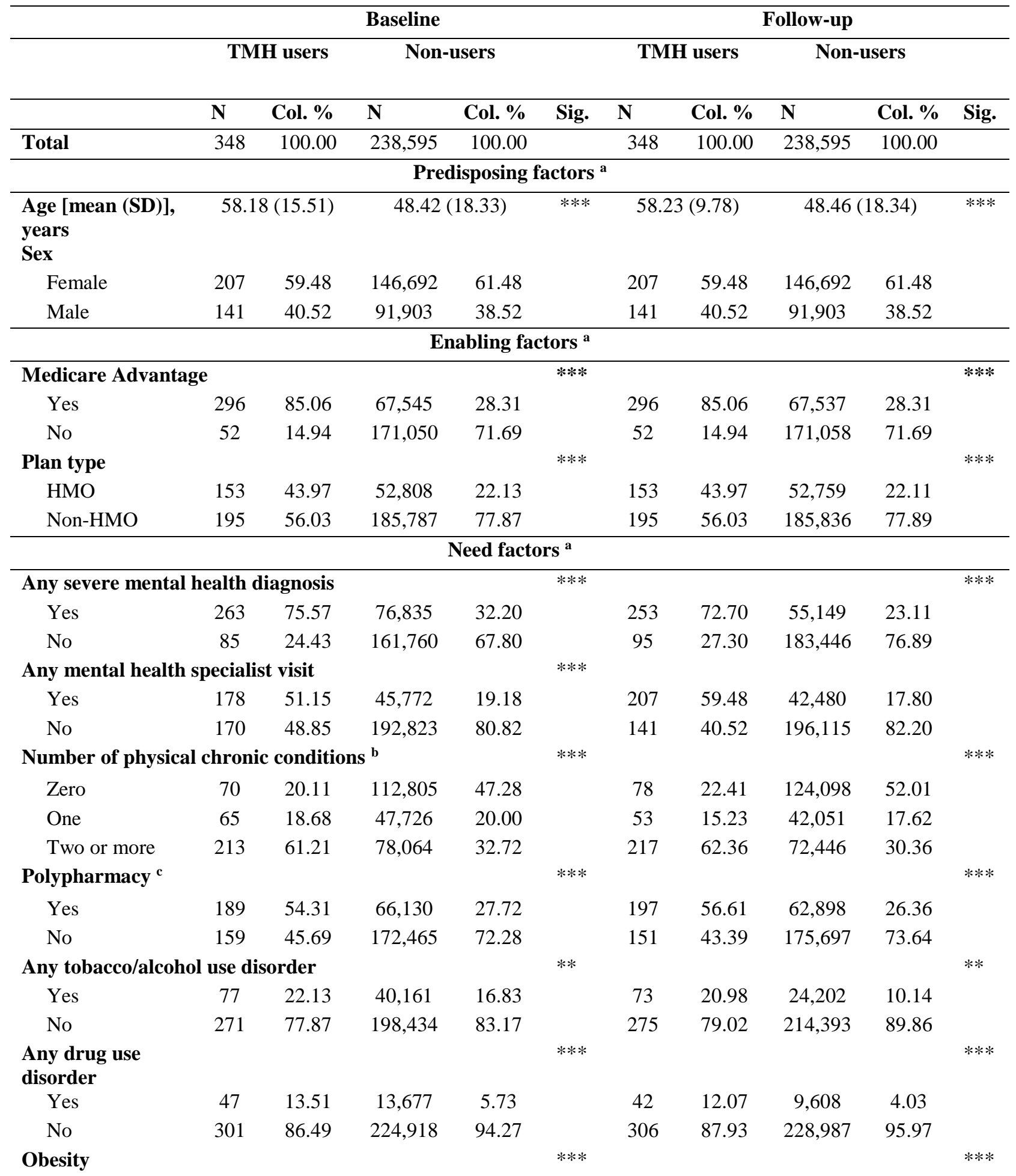




\begin{tabular}{|c|c|c|c|c|c|c|c|c|c|c|}
\hline Yes & 57 & 16.38 & 19,235 & 8.06 & & 47 & 13.51 & 16,571 & 6.95 & \\
\hline No & 291 & 83.62 & 219,360 & 91.94 & & 301 & 86.49 & 222,024 & 93.05 & \\
\hline \multicolumn{11}{|c|}{ Environmental factors } \\
\hline Poverty $^{d}$ & & & & & $* *$ & & & & & $* *$ \\
\hline $\begin{array}{l}\text { Low (1st \& 2nd } \\
\text { Quartile) }\end{array}$ & 165 & 47.41 & 92,997 & 38.93 & & 165 & 47.41 & 92,997 & 38.93 & \\
\hline $\begin{array}{l}\text { High (3rd \& 4th } \\
\text { Quartile) }\end{array}$ & 183 & 52.59 & 145,869 & 61.07 & & 183 & 52.59 & 145,869 & 61.07 & \\
\hline Rurality $^{d}$ & & & & & $* * *$ & & & & & $* * *$ \\
\hline $\begin{array}{l}\text { Low (1st \& 2nd } \\
\text { Quartile) }\end{array}$ & 174 & 50.00 & 149,879 & 62.75 & & 174 & 50.00 & 149,879 & 62.75 & \\
\hline $\begin{array}{l}\text { High (3rd \& 4th } \\
\text { Quartile) }\end{array}$ & 174 & 50.00 & 88,987 & 37.25 & & 174 & 50.00 & 88,987 & 37.25 & \\
\hline \multicolumn{5}{|c|}{ Mental health provider shortage ${ }^{d}$} & $* * *$ & & & & & $* * *$ \\
\hline $\begin{array}{l}\text { Low (1st \& 2nd } \\
\text { Quartile) }\end{array}$ & 101 & 29.02 & 130,580 & 54.67 & & 101 & 29.02 & 130,580 & 54.67 & \\
\hline $\begin{array}{l}\text { High (3rd \& 4th } \\
\text { Quartile) }\end{array}$ & 247 & 70.98 & 108,286 & 45.33 & & 247 & 70.98 & 108,286 & 45.33 & \\
\hline TMH capacity e & & & & & $* * *$ & & & & & $* * *$ \\
\hline 1st Quartile & 22 & 6.32 & 62,555 & 26.19 & & 22 & 6.32 & 62,555 & 26.19 & \\
\hline 2nd Quartile & 86 & 24.71 & 72,528 & 30.36 & & 86 & 24.71 & 72,528 & 30.36 & \\
\hline 3rd Quartile & 145 & 41.67 & 48,624 & 20.36 & & 145 & 41.67 & 48,624 & 20.36 & \\
\hline 4th Quartile) & 95 & 27.30 & 55,159 & 23.09 & & 95 & 27.30 & 55,159 & 23.09 & \\
\hline $\begin{array}{l}\text { Lagging time } \\
\text { [mean (SD)], days }\end{array}$ & \multicolumn{2}{|c|}{$179.0(105.0)$} & \multicolumn{2}{|c|}{$136.2(104.4)$} & $* * *$ & 179. & 105.0) & \multicolumn{2}{|c|}{$136.2(104.4)$} & $* * *$ \\
\hline
\end{tabular}

Note: TMH users were defined as adults with MHC used any TMH services; non-users were defined as adults with MHC who only used in-person MH services.

Col.\%: Column\%; SD: standard deviation; Sig.: significance level;

HMO: health maintenance organization;

${ }^{a}$ all patient-level factors were measured in the six-month period before and after index date based on de-identified claims of Optum Clinformatics ${ }^{\circledR}$ DataMart. The first TMH use date was used as index date for users and a pseudoindex date was randomly selected from all mental health service dates among non-users.

${ }^{\mathrm{b}}$ chronic conditions examined included arthritis, asthma, coronary artery disease, cardiac arrhythmias, congestive heart failure, chronic kidney disease, chronic obstructive pulmonary disease, dementia and related disorders, diabetes, hypertension, hyperlipidemia, osteoporosis, stroke.

${ }^{c}$ polypharmacy was defined as having five or more medications from different therapeutic classes in the 90 days before index date.

${ }^{\mathrm{d}}$ poverty, rurality, and mental health provider shortage were measured as average percentages of counties under qualified for each condition from 2010-2016 based on data from the 2016-2017 Area Health Resources File.

${ }^{\mathrm{e}} \mathrm{TMH}$ capacity was measured as average percentages of mental health facilities with telemedicine from 2010-2016 based on data from the 2010-2016 National Mental Health Services Survey.

${ }^{\mathrm{f}}$ lagging time reflects the number of days between first observed MHC diagnosis in claims to index date/pseudoindex date.

${ }^{* *}, \mathrm{p}<0.01 ;{ }^{* * *}, \mathrm{p}<0.001$. 
Table 4.2 Unadjusted mean and Standard Deviation (SD) of baseline and follow-up costs (2018 US \$) of adults with mental health conditions (MHC) who used Telemental health services and those used in-person mental health (MH) services, Optum Clinformatics ${ }^{\circledR}$ DataMart, 2010-2017

\begin{tabular}{|c|c|c|c|c|c|c|c|c|c|c|}
\hline \multicolumn{11}{|c|}{ All adults with MHC } \\
\hline & \multicolumn{5}{|c|}{ TMH users } & \multicolumn{5}{|c|}{ Non-users } \\
\hline & \multicolumn{2}{|c|}{ Baseline } & \multicolumn{2}{|c|}{ Follow-up } & \multirow[t]{2}{*}{ Sig. } & \multicolumn{2}{|c|}{ Baseline } & \multicolumn{2}{|c|}{ Follow-up } & \multirow[t]{2}{*}{ Sig. } \\
\hline & $\mathbf{N}$ & $\begin{array}{l}\text { Mean } \\
\text { (SD) }\end{array}$ & $\mathbf{N}$ & $\begin{array}{l}\text { Mean } \\
\text { (SD) }\end{array}$ & & $\mathbf{N}$ & $\begin{array}{l}\text { Mean } \\
\text { (SD) }\end{array}$ & $\mathbf{N}$ & $\begin{array}{l}\text { Mean } \\
(\text { SD) }\end{array}$ & \\
\hline Total $^{\text {a }}$ & 348 & $\begin{array}{c}12,960.56 \\
(24,260.72)\end{array}$ & 348 & $\begin{array}{c}13,336.58 \\
(26,420.70)\end{array}$ & ns & 238,595 & $\begin{array}{c}6,642.34 \\
(23,519.44)\end{array}$ & 238,595 & $\begin{array}{c}6,115.61 \\
(21,312.88)\end{array}$ & $* * *$ \\
\hline $\begin{array}{l}\text { MH- } \\
\text { related }\end{array}$ & 348 & $\begin{array}{c}2,927.83 \\
(10.980 .45)\end{array}$ & 348 & $\begin{array}{c}3,669.71 \\
(11,899.49)\end{array}$ & ns & 238,595 & $\begin{array}{c}1,035.34 \\
(6,775.67)\end{array}$ & 238,595 & $\begin{array}{c}735.94 \\
(6,091.44)\end{array}$ & $* * *$ \\
\hline \multicolumn{11}{|c|}{ Adults with MHC with specific service use } \\
\hline & \multicolumn{5}{|c|}{ TMH users } & \multicolumn{5}{|c|}{ Non-users } \\
\hline & \multicolumn{2}{|c|}{ Baseline } & \multicolumn{2}{|c|}{ Follow-up } & Sig. & \multicolumn{2}{|c|}{ Baseline } & \multicolumn{2}{|c|}{ Follow-up } & Sig. \\
\hline & $\mathbf{N}$ & $\begin{array}{l}\text { Mean } \\
\text { (SD) }\end{array}$ & $\begin{array}{l}\mathrm{N} \\
(\%)\end{array}$ & $\begin{array}{l}\text { Mean } \\
\text { (SD) }\end{array}$ & & $\mathrm{N}(\%)$ & $\begin{array}{l}\text { Mean } \\
\text { (SD) }\end{array}$ & $\mathrm{N}(\%)$ & $\begin{array}{l}\text { Mean } \\
\text { (SD) }\end{array}$ & \\
\hline Total $^{\text {a }}$ & 327 & $\begin{array}{c}13792.89 \\
(24,798.83)\end{array}$ & 328 & $\begin{array}{c}14149.79 \\
(27,003.81)\end{array}$ & ns & 221,638 & $\begin{array}{c}7,150.53 \\
(23946.90)\end{array}$ & 219,822 & $\begin{array}{c}6,637.88 \\
(22,126.10)\end{array}$ & $* * *$ \\
\hline $\begin{array}{l}\text { MH- } \\
\text { related b }\end{array}$ & 105 & $\begin{array}{c}9,703.66 \\
(18,327.71)\end{array}$ & 116 & $\begin{array}{c}11,009.12 \\
(18,594.44)\end{array}$ & $\mathrm{ns}$ & 33,304 & $\begin{array}{c}7,417.31 \\
(16,780.17)\end{array}$ & 19,742 & $\begin{array}{c}8,894.29 \\
(19,388.17)\end{array}$ & $* * *$ \\
\hline
\end{tabular}

Note: All outcomes were measured based on de-identified claims of Optum Clinformatics ${ }^{\circledR}$ DataMart. All the costs were standardized to 2018 US dollar. Baseline and follow-up period were defined as the six-month period before and after index/pseudo-index date, respectively. The first TMH use date was used as index date for users and a pseudo-index date was randomly selected from all mental health service dates among non-users.

TMH users were defined as adults with MHC used any TMH services; non-users were defined as adults with MHC who only used in-person MH services.

${ }^{a}$ total healthcare costs included the standard costs for all healthcare services paid by payers, including those for outpatient visits, inpatient stays, and prescription medications.

${ }^{\mathrm{b}} \mathrm{MH}$-related utilizations and costs were identified based on primary/secondary diagnoses of any MHC.

c $\% \mathrm{MH}$ costs were calculated as the average percentage of $\mathrm{MH}$-related costs among the total healthcare costs for individuals with positive $\mathrm{MH}-$ related costs.

$\mathrm{ns}, \mathrm{p}>0.05$; $^{* * *}, \mathrm{p}<0.001$. 
Table 4.3 Parameter estimates of Telemental health use (TMH) from difference-in-differences (DID) analyses for total healthcare costs and total costs for mental health services (MHS) at follow-up among adults with mental health conditions (MHC), Optum Clinformatics ${ }^{\circledR}$ DataMart, 2010-2017

\begin{tabular}{|c|c|c|c|}
\hline \multicolumn{4}{|c|}{ Total healthcare costs ${ }^{a}$} \\
\hline & Logistic & GLM & Marginal effect \\
\hline & AOR (95\% CI) & Adjusted Beta (SE) & Mean $(95 \% \mathrm{CI}), \$$ \\
\hline \multicolumn{4}{|l|}{ TMH use } \\
\hline Yes vs. No & $1.06(0.68,1.66)$ & $-0.043(0.104)$ & $-253.9(-1,601.5,1,093.7)$ \\
\hline \multicolumn{4}{|l|}{ Time } \\
\hline Follow-up vs. baseline & $0.89(0.88,0.91)^{* * *}$ & $0.023(0.009)^{* * *}$ & $-197.1(-307.0,-87.2)^{* * *}$ \\
\hline DID $^{c}$ & $1.18(0.70,2.00)$ & $0.074(0.121)$ & $554.3(-1,005.2,2,113.8)$ \\
\hline \multicolumn{4}{|c|}{ Total MH-related costs ${ }^{b}$} \\
\hline & Logistic & GLM & Marginal effect \\
\hline & AOR $(95 \%$ CI $)$ & Adjusted Beta (SE) & Mean $(95 \% \mathrm{CI}), \$$ \\
\hline \multicolumn{4}{|l|}{ TMH use } \\
\hline Yes vs. No & $1.84(1.44,2.34)$ & $0.130(0.203)$ & $461.3(142.4,780.2)^{* * *}$ \\
\hline \multicolumn{4}{|l|}{ Time } \\
\hline Follow-up vs. baseline & $0.56(0.55,0.57)^{* * *}$ & $0.108(0.016)^{* * *}$ & $-269.9(-294.3,-245.5)^{* * *}$ \\
\hline DID $^{c}$ & $2.06(1.62,2.63)^{* * *}$ & $-0.097(0.232)$ & $369.6(13.9,725.4)^{*}$ \\
\hline
\end{tabular}

Note: All outcomes were measured based on de-identified claims of Optum Clinformatics ${ }^{\circledR}$ DataMart. All the costs were standardized to 2018 US dollar. Baseline and follow-up period were defined as the six-month period before and after index/pseudo-index date, respectively. The first TMH use date was used as index date for users and a pseudo-index date was randomly selected from all mental health service dates among non-users.

AOR (95\%CI): adjusted odds ratio and associated 95\% confidence interval were estimated from logistic regressions on the probability of non-zero costs.

SE: standard error was estimated from two-part models, where the first part estimated the probabilities of having non-zero costs in a logistic regression and the second part estimated positive costs in a generalized linear model (GLM) with log-link functions and gamma distributions.

${ }^{a}$ Total healthcare costs included the standard costs for all healthcare services paid by payers, including those for outpatient visits, inpatient stays, and prescription medications.

${ }^{\mathrm{b}}$ Total costs for mental health services were identified based on primary/secondary diagnoses of any MHC from outpatient and inpatient claims.

${ }^{\mathrm{c}}$ DID is the interaction term of TMH use and Time, representing the impact of TMH use on the changes of outcomes from baseline to follow-up.

${ }^{* *}, \mathrm{p}<0.01 ;{ }^{* * *}, \mathrm{p}<0.001$. 


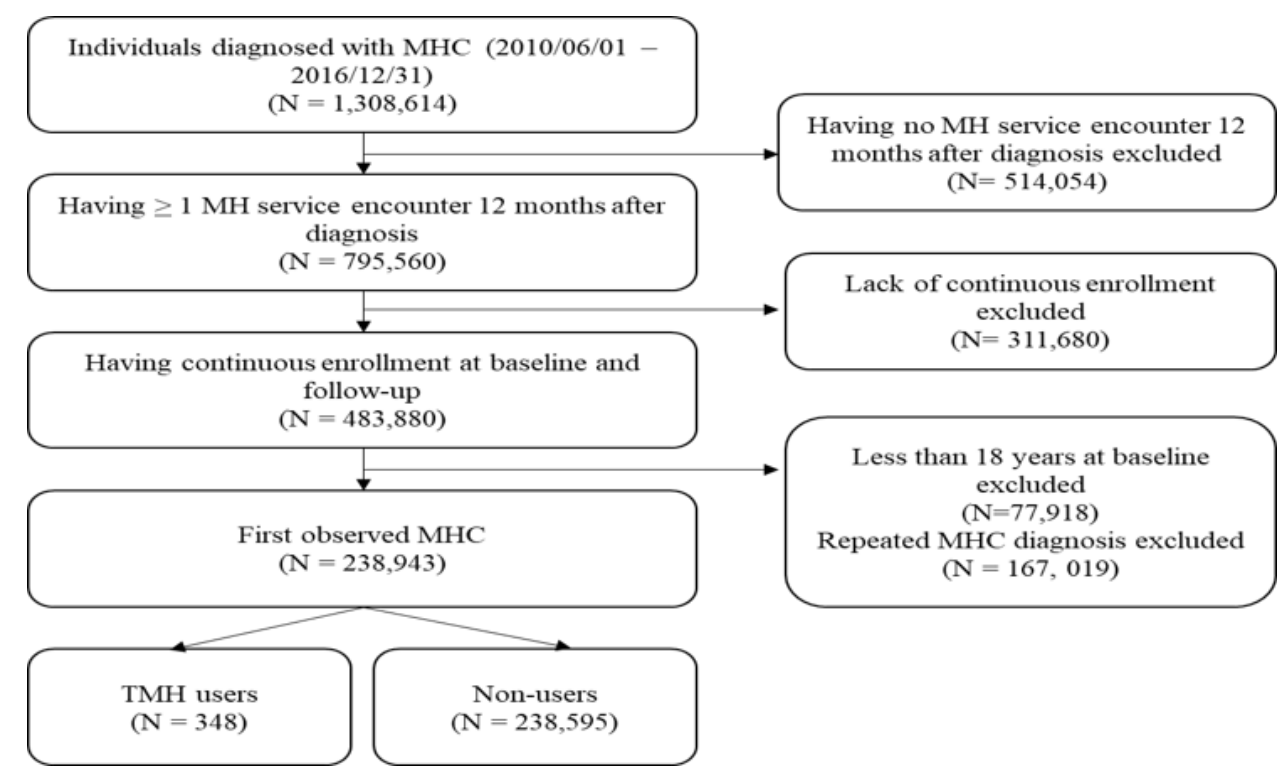

Figure 4.1 Aim 3 Cohort identification process. 


\section{CHAPTER 5}

\section{Summary and Conclusion}

\subsection{Summary}

Mental health was among the first clinical areas where telehealth technologies were applied to improve access to care. ${ }^{147}$ Understanding the adoption of TMH among mental health facilities is a critical step in the broad implementation of TMH in the delivery system of mental healthcare. Examining facility- and state-level factors that may influence facility-level decisions to invest in TMH can inform federal and state-level policy initiatives and aid in improving the adoption of TMH by facilities. As the use of TMH is determined by not only "supply" but also "demand," it is also important to understand TMH use among adults with MHC. Examining TMH use among patients with MHC can establish benchmarks for demand in TMH and suggest future directions for expanding TMH services. In alignment with the "Triple Aim," the National Quality Forum (NQF) and the American Telemedicine Association use healthcare costs as one of the critical measurements in their newly developed telehealth value framework. ${ }^{62}$ Therefore, evaluating how TMH use influences healthcare utilization and costs among adults with MHC can establish the value of TMH services, which will, in turn, help inform and guide healthcare payers' decisions of expanding TMH coverage. ${ }^{67}$

However, a comprehensive examination of the adoption and impact of TMH in the realworld clinical practice setting is lacking. Given that commercial insurances plays an important

role in paying for mental health services and telehealth services, ${ }^{38,48}$ it is imperative to examine TMH use and the impact of TMH use on healthcare costs among commercially insured adults with MHC. Therefore, this dissertation pursued three related aims: 1) to estimate TMH adoption and examine factors associated with TMH adoption among mental health facilities; 2) to 
characterize TMH use and identify factors associated with TMH use among commercially insured adults with MHC; and 3) to evaluate how TMH use influence healthcare costs. To comprehensively present the state of art of TMH in the current mental health delivery system, the study used diverse data sources. These data sources included: the National Mental Health Services Survey (N-MHSS) for years 2011-2016; a geographically diverse claims database for commercially insured adults for years 2010-2017; the latest Area Health Resources File (20162017); and national reports of telehealth policies.

\section{TMH Adoption among Mental Health Facilities}

We found that about one in four mental health facilities in the US incorporated TMH as a treatment approach by 2016. The adoption of TMH by a mental health facility could be influenced by multiple facility- and state-level factors. TMH adoption was more likely among facilities that were part of the VHA system, included outpatient settings, had public ownership, had comprehensive MH treatments or Quality Improvement practices, had Information Technology (IT) capacities, had private or non-Medicaid public payers, and those treating elderly patients. On the other hand, we found that TMH adoption was less likely among mental health facilities treating more African Americans or patients with substance abuse disorders. State-level factors associated with TMH adoption included: having special telehealth licensure for crossstate telehealth practices and having a high percentage of rural counties. Our findings suggest that TMH adoption among mental health facilities may be discouraged by the legal/regulatory burden on providers in seeking licenses for cross-state practice and by low IT capacity. In addition, our findings suggest there may also be racial disparities in TMH access and missed opportunities for utilizing TMH to treat substance abuse disorders.

\section{TMH Use among Commercially Insured Adults with MHC}


Approximately 16 in 10,000 adults with MHC and 47 in 10,000 of those enrolled in Medicare Advantage (MA) in underserved areas used TMH. Most TMH services were delivered by psychiatrists. TMH has mostly been used for consultations and pharmacological management. Psychotherapy delivered via TMH was rare relative to that delivered in-person. Patient characteristics associated with TMH use included: younger age, having higher monthly out-ofpocket expenditures, past mental health specialist visits, and diagnosis of a severe MHC. TMH use varied widely across states and was positively associated with high levels of shortages in mental health providers and with high TMH capacity of mental health facilities in the state. Our results suggest that current TMH use among adults with MHC was primarily driven by a need for accessing mental health specialists. Expanding TMH capacities among mental health facilities could help alleviate the unmet need for MH services.

\section{Impact of TMH use on Healthcare Costs}

We found that TMH use was associated with a greater likelihood of using MH services and with significantly higher MH-related costs. Furthermore, using any TMH service was associated with a greater increase in MH-related costs from baseline to follow-up as compared to having only in-person MH service use. However, TMH use did not have any significant impact on total third-party healthcare costs despite increased $\mathrm{MH}$-related costs. Our findings suggest that TMH use may help increase access to MH services without raising total healthcare costs.

\subsection{Implications}

This dissertation research revealed that facility-level IT capacity and state licensure policies/requirements for cross-state telehealth practice played essential roles in shaping the likelihood of TMH adoption among mental health facilities. Furthermore, the study findings suggested a positive association between TMH adoption by mental health facilities and TMH use 
among adults with MHC. These findings have important implications for state policymakers. As of 2017, medical boards in nine states had established processes to issue telehealth-specific licenses to allow out-of-state providers to provide telehealth services,${ }^{77}$ and twenty-two states had adopted the Federation of State Medical Board's Interstate Medical Licensure Compact to allow an expedited process of applying for licenses in other states. ${ }^{77}$ Similar policy initiatives that can reduce mental health providers' legal/regulatory burden of adopting TMH are needed to increase TMH capacity and thereafter encourage TMH use among adults with MHC.

Our study findings also have implications for state and federal funding agencies. As early development of TMH requires capital funds and infrastructure development, ${ }^{148}$ funding agencies need to be aware of the challenges faced by mental health facilities. Planning and securing funding for facilities that are in the early stage of developing a TMH system is critical to building TMH capacities.

The study findings also have implications for mental health facilities and mental health providers. We found that TMH adoption was more likely among mental health facilities with higher patient volumes and comprehensive MH treatments. It has been reported that telehealth technology can improve physician efficiency by reducing waiting times and missed appointment. ${ }^{136,149,150}$ Mental health facilities and providers aiming to increase their care capacity may plan to implement TMH in their practice. However, for a TMH program to be successful and beneficial to the operation, mental health facilities and providers need to coordinate the volume and scheduling of TMH services with in-person services. ${ }^{151}$ Furthermore, mental health facilities and providers need to keep up with proper training and guidelines to increase clinicians' comfort and confidence in delivering various $\mathrm{MH}$ services via TMH. ${ }^{114,118}$ 
Currently, the majority of TMH services were delivered by psychiatrists. Additional research is needed to explore how to increase TMH use by allied mental health providers such as psychologists, social workers, counselors, and nurse practitioners, who play important roles in providing in-person mental healthcare. Pharmacological management is an essential part of mental healthcare. Given the increasingly important role that pharmacists are playing in comprehensive medication management in collaborative care models, future research needs to explore the feasibility, acceptability, and outcomes of pharmacist-delivered, TMH based medication management. ${ }^{116}$

Our findings can inform TMH-related policymaking by payers and other stakeholders. The current healthcare system is transforming patient-centered care to meet the "Triple Aim" goals for improving patient experience, population health, and reducing the cost of care ${ }^{61} \mathrm{TMH}$ offers a unique opportunity to achieve patient-centered care by integrating mental and physical health in primary care settings. ${ }^{138}$ Our results that TMH use could increase access to MH services without having a significant impact on total healthcare costs provide initial evidence of the value of TMH for the decisions of TMH expansion.

Although the study was not able to illustrate the mechanism, the study findings indicated that the costs of increased MH service use might be offset by potential cost-savings from improved physical health. Future studies should examine the sources of this apparent "costoffset" and explore whether TMH use can lead to cost-savings over a longer period. Furthermore, although TMH provides a viable mechanism of mental health integration, how to incorporate TMH into existing in-person care to maximize access to care is still under discussion. Several care models have been piloted, including direct service models, consultation 
models, and collaborative care models. ${ }^{138}$ Further research is needed to explore the best care model of incorporating TMH into primary care.

\subsection{Unique Contributions of this Study}

This study filled a critical knowledge gap. To our knowledge, this is the first populationbased study that comprehensively examined TMH adoption and associated facility- and statelevel factors among mental health facilities in the US. Our study findings offer insight into factors that may influence facility-level decisions to invest in TMH and suggest potential strategies to increase adoption. This is also the first study to evaluate the impact of TMH use on total third-party healthcare costs and MH-related costs among commercially insured adults with MHC. The findings provide initial real-world evidence for the value of TMH services in facilitating the delivery of MH services, which can inform healthcare payers' decision regarding TMH expansion. By examining how TMH is used in delivering various $\mathrm{MH}$ services and which

providers and patients use TMH most, our findings suggest directions for healthcare insurers and policymakers to better incorporate $\mathrm{TMH}$ in the current $\mathrm{MH}$ delivery system.

\subsection{Strengths and Limitations}

The strengths of this study include the use of diverse data sources to investigate the current state of TMH delivery, the use of population-based, linked nation-wide data that permits the examination of various multi-level factors, the use of robust statistical techniques to adjust for observed and unobserved selection bias, the use of a pre-post nonequivalent controlled group design to assess the comparability of costs across study groups, and the use of standardized prices of medical services and prescription medications to allow comparison of $\mathrm{MH}$ care costs across different geographical areas. 
Some limitations should also be noted. First, we estimated TMH adoption based on the reported telemedicine use among mental health facilities. Due to the lack of data on how telemedicine was used, we may have overestimated TMH adoption and use among mental health facilities in the US. Second, we only have the data to measure environmental factors and market characteristics at the state level and were therefore unable to capture within-state variation in these factors. Third, the results may be affected by unmeasured factors that were not captured in claims data, such as disease severity, race/ethnicity, education levels, and patient and provider preferences. Furthermore, subgroup analyses of individual MHC's or specific MH services were not possible due to small sample sizes of TMH users in these groups. Finally, the study findings of aim 2 and aim 3 may not be generalizable to adults with MHC who are insured by other commercial insurances.

\subsection{Conclusion}

Although TMH appears to offer a promising tool for facilities to expand access to care and serve high-need patients, only one in four mental health facilities had adopted TMH in 2016. TMH adoption might be hindered by low facility-level information technology capacity and state licensure requirements for cross-state telehealth practice. TMH use among adults with MHC was low in this study of a commercially insured population. Current TMH use among adults with MHC was driven mainly by a greater need to access mental health specialists. Collaborative efforts by policymakers, payers, and providers, particularly those who could expand TMH capacity in healthcare settings, are needed to increase the availability of TMH in mental health care delivery, and thereby increase access to mental healthcare services for adults with MHC. TMH can increase access to MH care without increasing total healthcare costs among adults with MHC. Further research is needed to confirm our study findings; specifically, studies are needed 
to examine how TMH use affects the costs of mental and physical care separately and to explore whether TMH use can lead to cost-savings over a longer period among adults with different MHC. 


\section{Reference}

1. Stein DJ, Phillips KA, Bolton D, Fulford KWM, Sadler JZ, Kendler KS. What is a Mental/Psychiatric Disorder? From DSM-IV to DSM-V. Psychological medicine. 2010;40(11):1759-1765.

2. Ahrnsbrak R, Bose J, Hedden S, Lipari R, Park-Lee E. Key substance use and mental health indicators in the United States: Results from the 2016 National Survey on Drug Use and Health. Center for Behavioral Health Statistics and Quality, Substance Abuse and Mental Health Services Administration: Rockville, MD, USA. 2017.

3. Vigo D, Thornicroft G, Atun R. Estimating the true global burden of mental illness. The Lancet Psychiatry. 2016;3(2):171-178.

4. Trautmann S, Rehm J, Wittchen HU. The economic costs of mental disorders: Do our societies react appropriately to the burden of mental disorders? EMBO reports. 2016:e201642951.

5. Cohon C, Manderscheid R. Congruencies in increased mortality rates, years of potential life lost, and causes of death among public mental health clients in eight states. Preventing Chronic Disease. 2006;3(2):1-14.

6. Druss BG, Zhao L, Von Esenwein S, Morrato EH, Marcus SC. Understanding excess mortality in persons with mental illness: 17-year follow up of a nationally representative US survey. Medical care. 2011:599-604.

7. Mental Health America. Mental health treatments. http://www.mentalhealthamerica.net/types-mental-health-treatments. Accessed July 4, 2018.

8. Kisely S, Scott A, Denney J, Simon G. Duration of untreated symptoms in common mental disorders: association with outcomes: international study. The British Journal of Psychiatry. 2006;189(1):79-80.

9. Lauren Gleason. Community Leaders Identify Mental Health System As One Of Most Overburdened Community Resources; Consequences Include Stressed Emergency Medical And Education Systems. National Alliance on Mental Illness; 2003.

10. Beronio K, Po R, Skopec L, Glied S. Affordable Care Act will expand mental health and substance use disorder benefits and parity protections for 62 million Americans. Mental Health. 2014;2.

11. Unützer J, Harbin H, Schoenbaum M, Druss B. The collaborative care model: An approach for integrating physical and mental health care in Medicaid health homes. Health Home Information Resource Center. 2013:1-13.

12. Clyburn ML. Broadband Prescriptions for Mental Health: A Policy Conference. Hous $J$ Health L \& Pol'y. 2017; 17:1.

13. Olfson M. Building the mental health workforce capacity needed to treat adults with serious mental illnesses. Health Affairs. 2016;35(6):983-990.

14. Kaiser Family Foundation. Mental Health Care Health Professional Shortage Areas (HSPAs). 2017; https://www.kff.org/other/state-indicator/mental-health-care-healthprofessional-shortage-areashpsas/?currentTimeframe $=0 \&$ sortModel $=\% 7 \mathrm{~B} \% 22$ colId $\% 22: \% 22$ Location $\% 22, \% 22$ sort \%22:\%22asc\%22\%7D. Accessed July 4, 2018. 
15. Konrad TR, Ellis AR, Thomas KC, Holzer CE, Morrissey JP. County-level estimates of need for mental health professionals in the United States. Psychiatric Services. 2009;60(10):1307-1314.

16. New study reveals root cause of mental health crisis. [press release]. Cohen Veterans Network2018.

17. Williams ME, Latta J, Conversano P. Eliminating the wait for mental health services. The journal of behavioral health services \& research. 2008;35(1):107-114.

18. America CoQoHCi, Staff IoM. Crossing the quality chasm: A new health system for the 21st century. National Academies Press; 2001.

19. Corrigan PW, Watson AC. Understanding the impact of stigma on people with mental illness. World psychiatry. 2002;1(1):16.

20. Baron RJ, Cassel CK. 21st-century primary care: New physician roles need new payment models. JAMA. 2008;299(13):1595-1597.

21. (ATA). ATA. Telemedicine glossary. http://thesource.americantelemed.org/resources/telemedicine-glossary. Accessed June 27, 2018.

22. Rogers MA, Buchan DA, Small D, Stewart CM, Krenzer BE. Telemedicine improves diagnosis of essential hypertension compared with usual care. Journal of Telemedicine and telecare. 2002;8(6):344-349.

23. Li Z, Wu C, Olayiwola J, Hilaire D, Huang J. Telemedicine-based digital retinal imaging vs standard ophthalmologic evaluation for the assessment of diabetic retinopathy. Connecticut medicine. 2012;76(2):85-90.

24. Shah NB, Der E, Ruggerio C, Heidenreich PA, Massie BM. Prevention of hospitalizations for heart failure with an interactive home monitoring program. American heart journal. 1998;135(3):373-378.

25. Bartoli L, Zanaboni P, Masella C, Ursini N. Systematic review of telemedicine services for patients affected by chronic obstructive pulmonary disease (COPD). Telemedicine and e-Health. 2009;15(9):877-883.

26. Hersh WR, Hickam DH, Severance SM, Dana TL, Krages KP, Helfand M. Diagnosis, access and outcomes: Update of a systematic review of telemedicine services. Journal of Telemedicine and Telecare. 2006;12(2_suppl):3-31.

27. Yellowlees P, Shore J, Roberts L. Practice guidelines for videoconferencing-based telemental health - October 2009. Telemedicine journal and e-health : the official journal of the American Telemedicine Association. 2010;16(10):1074-1089.

28. Grady B, Myers KM, Nelson E-L, et al. Evidence-based practice for telemental health. Telemedicine and e-Health. 2011;17(2):131-148.

29. Hubley S, Lynch SB, Schneck C, Thomas M, Shore J. Review of key telepsychiatry outcomes. World Journal of Psychiatry. 2016;6(2):269-282.

30. Mechanic D. Removing barriers to care among persons with psychiatric symptoms. Health Affairs. 2002;21(3):137-147.

31. May C, Gask L, Atkinson T, Ellis N, Mair F, Esmail A. Resisting and promoting new technologies in clinical practice: the case of telepsychiatry. Social science \& medicine. 2001;52(12):1889-1901.

32. Fortney JC, Pyne JM, Turner EE, et al. Telepsychiatry integration of mental health services into rural primary care settings. International Review of Psychiatry.

2015;27(6):525-539. 
33. Legislatures NCoS, Legislatures FfS. Telehealth: Policy Trends and Considerations. 2015.

34. Adam D. The Growth of Telehealth Services in the Veterans Health Administration Between 1994 and 2014: A Study in the Diffusion of Innovation. Telemedicine and eHealth. 2014;20(9):761-768.

35. Gilman M, Stensland J. Telehealth and Medicare: Payment Policy, Current Use, and Prospects for Growth. Medicare \& Medicaid Research Review. 2013;3(4):mmrr.003.004.a004.

36. The Centers for Medicare \& Medicaid Services (CMS). Telehealth services. . 2018.

37. Thomas L CG. 50 State telemedicine gap analysis: coverage \& reimbursement. . American Telemedicine Association;2017.

38. Robert E. Moffit; Ben Steffen. Office-based physician adoption of telehealth. Maryland health care commission 2018.

39. Adler-Milstein J, Kvedar J, Bates DW. Telehealth among US hospitals: several factors, including state reimbursement and licensure policies, influence adoption. Health Affairs. 2014;33(2):207-215.

40. Center for Connected Health Policy. State telehealth laws and medicaid program policies: A comprehensive scan of the 50 states and District of Columbia. 2015.

41. Douglas MD, Xu J, Heggs A, Wrenn G, Mack DH, Rust G. Assessing telemedicine utilization by using Medicaid claims data. Psychiatric Services. 2016;68(2):173-178.

42. Mehrotra A, Huskamp HA, Souza J, et al. Rapid growth in mental health telemedicine use among rural Medicare beneficiaries, wide variation across states. Health Affairs. 2017;36(5):909-917.

43. Mehrotra A, Jena AB, Busch AB, Souza J, Uscher-Pines L, Landon BE. Utilization of telemedicine among rural Medicare beneficiaries. Jama. 2016;315(18):2015-2016.

44. Wilson FA, Rampa S, Trout KE, Stimpson JP. Telehealth Delivery of Mental Health Services: An Analysis of Private Insurance Claims Data in the United States. Psychiatric services (Washington, DC). 2017;68(12):1303-1306.

45. Rowan K, McAlpine DD, Blewett LA. Access and cost barriers to mental health care, by insurance status, 1999-2010. Health affairs (Project Hope). 2013;32(10):1723-1730.

46. Andrade LH, Alonso J, Mneimneh Z, et al. Barriers to mental health treatment: results from the WHO World Mental Health surveys. Psychological Medicine. 2013;44(6):13031317.

47. Goldberg JF, Kurita K, Hoblyn J, et al. Depressive illness burden associated with complex polypharmacy in patients with bipolar disorder: findings from the STEP-BD. The Journal of clinical psychiatry. 2009;70(2):155-162.

48. Mental Health Services Abuse Substance Administration. 2015 National Survey on Drug Use and Health. 2016.

49. UnitedHealthcare. Telehealth and Telemedicine Policy, Professional. 2019.

50. Kasckow J, Felmet K, Appelt C, Thompson R, Rotondi A, Haas G. Telepsychiatry in the assessment and treatment of schizophrenia. Clinical schizophrenia \& related psychoses. 2013;8(1):21-27A.

51. Norman S. The use of telemedicine in psychiatry. Journal of psychiatric and mental health nursing. 2006;13(6):771-777.

52. Langarizadeh M, Tabatabaei MS, Tavakol K, Naghipour M, Rostami A, Moghbeli F. Telemental Health Care, an Effective Alternative to Conventional Mental Care: a 
Systematic Review. Acta informatica medica : AIM : journal of the Society for Medical Informatics of Bosnia \& Herzegovina : casopis Drustva za medicinsku informatiku BiH. 2017;25(4):240-246.

53. Hilty DM, Ferrer DC, Parish MB, Johnston B, Callahan EJ, Yellowlees PM. The effectiveness of telemental health: a 2013 review. Telemedicine journal and e-health : the official journal of the American Telemedicine Association. 2013;19(6):444-454.

54. Bashshur RL, Shannon GW, Bashshur N, Yellowlees PM. The empirical evidence for telemedicine interventions in mental disorders. Telemedicine and e-Health. 2016;22(2):87-113.

55. Carter RE, Haynes LF, Back SE, et al. Improving the transition from residential to outpatient addiction treatment: Gender differences in response to supportive telephone calls. The American journal of drug and alcohol abuse. 2008;34(1):47-59.

56. Deen TL, Fortney JC, Pyne JM. Relationship between satisfaction, patient-centered care, adherence and outcomes among patients in a collaborative care trial for depression. Administration and Policy in Mental Health and Mental Health Services Research. 2011;38(5):345-355.

57. Piette JD, Sussman JB, Pfeiffer PN, Silveira MJ, Singh S, Lavieri MS. Maximizing the value of mobile health monitoring by avoiding redundant patient reports: Prediction of depression-related symptoms and adherence problems in automated health assessment services. Journal of medical internet research. 2013;15(7).

58. Kasckow J, Felmet K, Appelt C, Thompson R, Rotondi A, Haas G. Telepsychiatry in the assessment and treatment of schizophrenia. Clinical schizophrenia \& related psychoses. 2014;8(1):21-27A.

59. D'Souza R. Improving treatment adherence and longitudinal outcomes in patients with a serious mental illness by using telemedicine. Journal of telemedicine and telecare. 2002;8(2_suppl):113-115.

60. Deen TL, Godleski L, Fortney JC. A description of telemental health services provided by the Veterans Health Administration in 2006-2010. Psychiatric Services. 2012;63(11):1131-1133.

61. Donald M. Berwick TWN, John Whittington,. The Triple Aim: Care, Health, And Cost. Health Affairs. 2008;27(3):759-769.

62. Frandsen BR, Joynt KE, Rebitzer JB, Jha AK. Care fragmentation, quality, and costs among chronically ill patients. The American journal of managed care. 2015;21(5):355362.

63. Smith AC, Scuffham P, Wootton R. The costs and potential savings of a novel telepaediatric service in Queensland. BMC health services research. 2007;7(1):35.

64. Rabinowitz T, Murphy KM, Amour JL, Ricci MA, Caputo MP, Newhouse PA. Benefits of a Telepsychiatry Consultation Service for Rural Nursing Home Residents. Telemedicine and e-Health. 2010;16(1):34-40.

65. Modai I, Jabarin M, Kurs R, Barak P, Hanan I, Kitain L. Cost effectiveness, safety, and satisfaction with video telepsychiatry versus face-to-face care in ambulatory settings. Telemedicine Journal \& E-Health. 2006;12(5):515-520.

66. Butler TN, Yellowlees P. Cost analysis of store-and-forward telepsychiatry as a consultation model for primary care. Telemedicine and e-Health. 2012;18(1):74-77. 
67. Congressional Budget Office. Answers to questions fo the record following a hearing by the house committee on the budget on the work of the Congressional Budget Office. Congressional Budget Office website July 29, 20152015.

68. Menachemi N, Burke DE, Ayers DJ. Factors Affecting the Adoption of Telemedicine-A Multiple Adopter Perspective. Journal of Medical Systems. 2004;28(6):617-632.

69. Kruse CS, DeShazo J, Kim F, Fulton L. Factors associated with adoption of health information technology: a conceptual model based on a systematic review. JMIR medical informatics. 2014;2(1).

70. Freedman S, Lin H. Hospital Ownership Type and Innovation: The Case of Electronic Medical Records Adoption. Nonprofit and Voluntary Sector Quarterly. 2018;47(3):537561.

71. Yuen EK, Goetter EM, Herbert JD, Forman EM. Challenges and opportunities in internet-mediated telemental health. Professional Psychology: Research and Practice. 2012;43(1):1.

72. Bartley M, Ferrie J, Montgomery S, Marmot M, Wilkinson R. Social determinants of health. The solid facts Copenhagen: Centre for Urban Health World Health Organization Regional Office for Europe. 2006:1-32.

73. Edmunds M, Tuckson R, Lewis J, et al. An emergent research and policy framework for telehealth. eGEMs. 2017;5(2).

74. Andersen RM. Revisiting the behavioral model and access to medical care: does it matter? Journal of health and social behavior. 1995:1-10.

75. Federal Communications Commission. Broadband progress report. Washington, DC: Federal Communications Commission. 2016.

76. Thomas L, Capistrant G. State telemedicine gaps analysis: coverage and reimbursement. American Telemedicine Association. 2015.

77. Center for Connected Health Policy. State Telehealth Laws and Reimbursement Policies: A Comprehensive Scan of the 50 States and District of Columbia. Public Health Institute Center for Connected Health Policy Sacramento, California; 2017.

78. Substance Abuse and Mental Health Services Administration. National Mental Health Services Survey (N-MHSS): 2016. Data on Mental Health Treatment Facilities. Substance Abuse and Mental Health Services Administration;2017.

79. Optum. Optum Clinformatics Data Mart[cited 2018 June 7].Available from: https://www.optum.com/content/dam/optum/resources/productSheets/Clinformatics_for_ Data_Mart.pdf. 2018.

80. Community Leaders Identify Mental Health System As One Of Most Overburdened Community Resources; Consequences Include Stressed Emergency Medical And Education Systems [press release]. National Alliance on Mental Illness, November 10, 20032003.

81. Deslich SA, Thistlethwaite T, Coustasse A. Telepsychiatry in correctional facilities: using technology to improve access and decrease costs of mental health care in underserved populations. The Permanente journal. 2013;17(3):80-86.

82. Post EP, Metzger M, Dumas P, Lehmann L. Integrating mental health into primary care within the Veterans Health Administration. Families, Systems, \& Health. 2010;28(2):83.

83. Shigekawa E, Fix M, Corbett G, Roby DH, Coffman J. The Current State Of Telehealth Evidence: A Rapid Review. Health Affairs. 2018;37(12):1975-1982.

84. US Department of Health Human Services. Area Health Resource File. 2017. 
85. Kane CK, Gillis K. The Use Of Telemedicine By Physicians: Still The Exception Rather Than The Rule. Health Affairs. 2018;37(12):1923-1930.

86. American Hospital Association. The promise of telehealth for hospitals, health systems and their communities. Washington, DC January. 2015.

87. Wilson LS, Maeder AJ. Recent Directions in Telemedicine: Review of Trends in Research and Practice. Healthcare informatics research. 2015;21(4):213-222.

88. VA announces Telemental Health Clinical Resource Centers during Telemedicine Association Gathering [press release]. May 16, 20162016.

89. National Academies of Sciences Engineering and Medicine. Evaluation of the Department of Veterans Affairs Mental Health Services. National Academies Press; 2018.

90. Walker ER, Cummings JR, Hockenberry JM, Druss BG. Insurance status, use of mental health services, and unmet need for mental health care in the United States. Psychiatric services (Washington, DC). 2015;66(6):578-584.

91. Horwitz JR. Making profits and providing care: comparing nonprofit, for-profit, and government hospitals. Health affairs. 2005;24(3):790-801.

92. S. BM, Lois K, Kathy T, et al. Implementing and Sustaining Team-Based Telecare for Bipolar Disorder: Lessons Learned from a Model-Guided, Mixed Methods Analysis. Telemedicine and e-Health. 2018;24(1):45-53.

93. Gellis ZD. Telemental health. Encyclopedia of geropsychology. 2015:1-6.

94. Kennedy BR, Mathis CC, Woods AK. African Americans and their distrust of the health care system: healthcare for diverse populations. Journal of cultural diversity. 2007;14(2):56-60.

95. George S, Hamilton A, Baker RS. How Do Low-Income Urban African Americans and Latinos Feel about Telemedicine? A Diffusion of Innovation Analysis. International journal of telemedicine and applications. 2012;2012:715194-715194.

96. National Alliance on Mental Illness. African American mental health. https://www.nami.org/Find-Support/Diverse-Communities/African-Americans. Accessed December 7, 2018.

97. Molfenter T, Boyle M, Holloway D, Zwick J. Trends in telemedicine use in addiction treatment. Addiction science \& clinical practice. 2015;10(1):14.

98. Eric Wicklund. Congress turns to Telemedicine to take on substance abuse epidemic. Telehealth news2018.

99. Prina LL. Foundation Funding To Improve Rural Health Care. Health affairs (Project Hope). 2016;35(1):174-175.

100. \$22.1 Million to Improve Access to Health Care in Rural Areas [press release]. HRSA website: HRSA NEWS ROOM, September 26, 20142014.

101. Diederich J, Song I. Mental health informatics: current approaches. Mental Health Informatics: Springer; 2014:1-16.

102. Roehrig C. Mental disorders top the list of the most costly conditions in the United States: \$201 billion. Health Affairs. 2016;35(6):1130-1135.

103. Park-Lee E, Lipari RN, Hedden SL, Kroutil L, Porter J. Receipt of services for substance use and mental health issues among adults: results from the 2016 National Survey on Drug Use and Health. 2018.

104. Thomas KC, Ellis AR, Konrad TR, Holzer CE, Morrissey JP. County-level estimates of mental health professional shortage in the United States. Psychiatric Services. 2009;60(10):1323-1328. 
105. Gamm L, Stone S, Pittman S. Mental health and mental disorders-A rural challenge: A literature review. Rural healthy people. 2010;1(1):97-114.

106. Clarke G, Yarborough BJ. Evaluating the promise of health IT to enhance/expand the reach of mental health services. General hospital psychiatry. 2013;35(4):339-344.

107. Zarate Jr CA, Weinstock L, Cukor P, et al. Applicability of telemedicine for assessing patients with schizophrenia: Acceptance and reliability. The Journal of clinical psychiatry. 1997.

108. Wallace PJ, Shah ND, Dennen T, Bleicher PA, Crown WH. Optum Labs: building a novel node in the learning health care system. Health Affairs. 2014;33(7):1187-1194.

109. Health Resources \& Services Administration (HRSA). Area Heatlh Resources Files. https://data.hrsa.gov/topics/health-workforce/ahrf. Accessed Jan 27, 2019.

110. Huskamp HA, Busch AB, Souza J, et al. How Is Telemedicine Being Used In Opioid And Other Substance Use Disorder Treatment? Health Affairs. 2018;37(12):1940-1947.

111. Masnoon N, Shakib S, Kalisch-Ellett L, Caughey GE. What is polypharmacy? A systematic review of definitions. BMC geriatrics. 2017;17(1):230-230.

112. Coffey RM, Kassed CA, Mhsa CS, Msw RV-w, Barrett ML. A severity-of-illness classification for mental and substance-use disorders for use with hospital administrative data. 2011.

113. Goodman RA, Posner SF, Huang ES, Parekh AK, Koh HK. Peer Reviewed: Defining and measuring chronic conditions: Imperatives for research, policy, program, and practice. Preventing chronic disease. 2013;10.

114. Shore JH, Yellowlees P, Caudill R, et al. Best Practices in Videoconferencing-Based Telemental Health April 2018. Telemedicine and e-Health. 2018;24(11):827-832.

115. Wittchen H-U, Mühlig S, Beesdo K. Mental disorders in primary care. Dialogues in clinical neuroscience. 2003;5(2):115-128.

116. Badowski ME, Walker S, Bacchus S, et al. Providing Comprehensive Medication Management in Telehealth. Pharmacotherapy: The Journal of Human Pharmacology and Drug Therapy. 2018;38(2):e7-e16.

117. Aboujaoude E, Salame W, Naim L. Telemental health: A status update. World psychiatry : official journal of the World Psychiatric Association (WPA). 2015;14(2):223-230.

118. Jenkins-Guarnieri MA, Pruitt LD, Luxton DD, Johnson K. Patient perceptions of telemental health: systematic review of direct comparisons to in-person psychotherapeutic treatments. Telemedicine and e-Health. 2015;21(8):652-660.

119. Simpson S. Psychotherapy via videoconferencing: A review. British Journal of Guidance \& Counselling. 2009;37(3):271-286.

120. Greenwald P, Stern ME, Clark S, Sharma R. Older adults and technology: in telehealth, they may not be who you think they are. International journal of emergency medicine. 2018;11(1):2-2.

121. Chi N-C, Demiris G. A systematic review of telehealth tools and interventions to support family caregivers. Journal of telemedicine and telecare. 2015;21(1):37-44.

122. Dullet NW, Geraghty EM, Kaufman T, et al. Impact of a University-Based Outpatient Telemedicine Program on Time Savings, Travel Costs, and Environmental Pollutants. Value in Health. 2017;20(4):542-546.

123. Russo JE, McCool RR, Davies L. VA Telemedicine: An Analysis of Cost and Time Savings. Telemed J E Health. 2016;22(3):209-215. 
124. Shannon GW, Bashshur RL, Lovett JE. Distance and the use of mental health services. The Milbank Quarterly. 1986;64(2):302-330.

125. Tseng KC, Hemenway D, Kawachi I, Subramanian SV, Chen WJ. Travel distance and the use of inpatient care among patients with schizophrenia. Administration and policy in mental health. 2008;35(5):346-356.

126. Flaum M. Telemental health as a solution to the widening gap between supply and demand for mental health services. New York, Elsevier; 2013.

127. Sheeran T, Rabinowitz T, Lotterman J, et al. Feasibility and impact of telemonitor-based depression care management for geriatric homecare patients. Telemedicine and e-Health. 2011;17(8):620-626.

128. Yuen EK, Herbert JD, Forman EM, et al. Acceptance based behavior therapy for social anxiety disorder through videoconferencing. Journal of Anxiety Disorders. 2013;27(4):389-397.

129. Hernandez-Tejada MA, Zoller JS, Ruggiero KJ, Kazley AS, Acierno R. Early Treatment Withdrawal from Evidence-Based Psychotherapy for PTSD: Telemedicine and in-Person Parameters. The International Journal of Psychiatry in Medicine. 2014;48(1):33-55.

130. E. RJ, R. MR, Louise D. VA Telemedicine: An Analysis of Cost and Time Savings. Telemedicine and e-Health. 2016;22(3):209-215.

131. Cota A, Tarchala M, Parent-Harvey C, et al. Review of 5.5 Years' Experience Using Email-Based Telemedicine to Deliver Orthopedic Care to Remote Communities. Telemedicine journal and e-health : the official journal of the American Telemedicine Association. 2017;23(1):37-40.

132. Hatcher-Martin J, Anderson E, Factor S. Patient Acceptance and Potential Cost-Savings of Teleneurology in an Academic Outpatient Movement Disorders Practice (P1.022). Neurology. 2016;86(16 Supplement).

133. Baser O. Modeling Healthcare Costs. STATinMED Research; 2012.

134. Lechner M. The estimation of causal effects by difference-in-difference methods. Foundations and Trends® in Econometrics. 2011;4(3):165-224.

135. Lustig TA. The role of telehealth in an evolving health care environment: workshop summary. National Academies Press; 2012.

136. Neufeld J, Case R. Walk-in telemental health clinics improve access and efficiency: a 2year follow-up analysis. Telemedicine journal and e-health : the official journal of the American Telemedicine Association. 2013;19(12):938-941.

137. Katon W, Unutzer J, Fan MY, et al. Cost-effectiveness and net benefit of enhanced treatment of depression for older adults with diabetes and depression. Diabetes care. 2006;29(2):265-270.

138. McWilliams JK. Integrating Telemental Healthcare with the Patient-Centered Medical Home Model. Journal of child and adolescent psychopharmacology. 2016;26(3):278282.

139. Kojima G, Bell C, Tamura B, et al. Reducing cost by reducing polypharmacy: the polypharmacy outcomes project. Journal of the American Medical Directors Association. 2012;13(9):818.e811-818.e818.818E815.

140. Tisha L. Deen, Ph.D. ,, Linda Godleski, M.D. , and, John C. Fortney, Ph.D. A Description of Telemental Health Services Provided by the Veterans Health Administration in 2006-2010. Psychiatric Services. 2012;63(11):1131-1133. 
141. Hommel KA, Hente E, Herzer M, Ingerski LM, Denson LA. Telehealth behavioral treatment for medication nonadherence: a pilot and feasibility study. European journal of gastroenterology \& hepatology. 2013;25(4):469-473.

142. Frangou S, Sachpazidis I, Stassinakis A, Sakas G. Telemonitoring of medication adherence in patients with schizophrenia. Telemedicine Journal \& E-Health. 2005;11(6):675-683.

143. Substance Abuse and Mental Health Services Administration. Key substance use and mental health indicators in the United States: Results from the 2016 National Survey on Drug Use and Health. Rockville, MD: Center for Behavioral Health Statistics and Quality, Substance Abuse and Mental Health Services Administration;2017.

144. Young LB. Telemedicine interventions for substance-use disorder: a literature review. Journal of Telemedicine and Telecare. 2012;18(1):47-53.

145. Spaulding R, Belz N, DeLurgio S, Williams AR. Cost savings of telemedicine utilization for child psychiatry in a rural Kansas community. Telemedicine and e-Health. 2010;16(8):867-871.

146. Wong J, Motulsky A, Abrahamowicz M, Eguale T, Buckeridge DL, Tamblyn R. Offlabel indications for antidepressants in primary care: descriptive study of prescriptions from an indication based electronic prescribing system. BMJ. 2017;356:j603.

147. Smith HA, Allison RA, Rockville M. Telemental health: Delivering mental health care at a distance. Unpublished summary report, US Department of Health and Human Services, Office for the Advancement of Telehealth, Rockville, MD. 1998.

148. Lambert D, Gale J, Hartley D, Croll Z, Hansen A. Understanding the Business Case for Telemental Health in Rural Communities. The Journal of Behavioral Health Services \& Research. 2016;43(3):366-379.

149. Sherling Dawn SM. The promises and pitfalls of telemedicine. The American Journal of Accountable Care. 2017.

150. Shore P, Goranson A, Ward MF, Lu MW. Meeting veterans where they're @: a VA Home-Based Telemental Health (HBTMH) pilot program. The International Journal of Psychiatry in Medicine. 2014;48(1):5-17.

151. Brooks E, Turvey C, Augusterfer EF. Provider barriers to telemental health: obstacles overcome, obstacles remaining. Telemedicine and e-Health. 2013;19(6):433-437. 


\section{Appendices}

\section{Appendix 7.1 Procedure codes and modifiers used to identify mental health visits.}

\begin{tabular}{|c|c|c|c|c|c|c|}
\hline 90791 & 90816 & W1027 & G0443 & H0046, HE & H5010 & 9411 \\
\hline 90792 & 90817 & W1030 & G0444 & H0047 & H5020 & 9412 \\
\hline 90785 & 90818 & W1032 & H0001 & H0048 & H5025 & 9413 \\
\hline 90832 & 90819 & W1033 & $\mathrm{H} 0001, \mathrm{HN}$ & H0049 & H5030 & 9419 \\
\hline 90833 & 90820 & W1035 & H0001, HO & H0050 & H540 & 9421 \\
\hline 90834 & 90821 & W1037 & H0001, TS & $\mathrm{H} 2000, \mathrm{HO}$ & $\mathrm{H} 550$ & 9422 \\
\hline 90836 & 90822 & W1038 & H0002 & H2000, HP & M0064 & 9423 \\
\hline 90837 & 90823 & W1039 & H0003 & $\mathrm{H} 2000$ & S0201 & 9425 \\
\hline 90838 & 90824 & W1044 & H0004 & H2010, HO & S5100 & 9429 \\
\hline 90839 & 90825 & W1046 & H0004, HR & H2010, HP & S5101 & 9431 \\
\hline 90840 & 90826 & W1048 & H0004, HF & H2010, HE & S5102 & 9432 \\
\hline 00104 & 90827 & W1049 & H0004, HG & H2010, HQ & S9475 & 9433 \\
\hline 9424 & 90828 & W1050 & H0004, HQ & H2010, HM & S9480 & 9434 \\
\hline 9426 & 90829 & W1059 & H0005 & H2010, HF & S9485 & 9435 \\
\hline 9427 & 90830 & W1061 & H0006 & $\mathrm{H} 2010$ & S9976 & 9436 \\
\hline 80100 & 90835 & W1064 & H0007 & $\mathrm{H} 2010, \mathrm{HN}$ & T1006 & 9437 \\
\hline 80101 & 90841 & W1065 & H0008 & H2011 & T1007, U8 & 9438 \\
\hline 80102 & 90842 & W1070 & H0009 & $\mathrm{H} 2012$ & T1007, SA & 9439 \\
\hline 80103 & 90843 & W1074 & H0010 & H2012, HF & T1007 & 9441 \\
\hline 80152 & 90844 & W1075 & H0011 & H2013 & T1007, TS & 9442 \\
\hline 80154 & 90845 & W9890 & H0012 & H2014 & T1008 & 9443 \\
\hline 80156 & 90846 & W9891 & H0013 & $\mathrm{H} 2015, \mathrm{HE}$ & $\mathrm{T} 1011$ & 9444 \\
\hline 80160 & 90847 & W9892 & H0014 & $\mathrm{H} 2015, \mathrm{HN}$ & T1012 & 9445 \\
\hline 80164 & 90848 & W9892 & H0015 & H2015, HQ & $\mathrm{T} 1015$ & 9446 \\
\hline 80166 & 90849 & G0071 & H0016 & H2015 & T1015, HE & 9449 \\
\hline 80173 & 90853 & G0072 & H0017 & H2016 & T1015, HF & 9451 \\
\hline 80174 & 90855 & G0073 & H0018 & H2017 & $\mathrm{T} 1015$ & 9452 \\
\hline 80178 & 90857 & G0074 & H0019 & $\mathrm{H} 2018$ & T1015, HE & 9453 \\
\hline 80182 & 90862 & G0075 & H0020 & H2019, HO & T1015 & 9454 \\
\hline 80201 & 90865 & G0076 & H0022 & H2019, HM & T1017 & 9455 \\
\hline 82055 & 90867 & G0077 & H0023 & H2019, HN & T1017, HA & 9459 \\
\hline 82075 & 90868 & G0078 & H0031 & H2019 & T1017, HB & 9461 \\
\hline 82145 & 90870 & G0079 & H0031, TS & H2019, HR & T1017, HK & 9462 \\
\hline 82205 & 90871 & G0080 & H0031, HA & H2019, HQ & T1017, TL & 9463 \\
\hline 82520 & 90872 & G0081 & $\mathrm{H} 0031, \mathrm{HO}$ & H2020 & T1023, HE & 9464 \\
\hline 82742 & 90875 & G0082 & H0031, TS & H2021 & T1023, HF & 9465 \\
\hline 83840 & 90876 & G0083 & $\mathrm{H} 0031, \mathrm{HN}$ & $\mathrm{H} 2022$ & $\mathrm{~T} 1025$ & 9466 \\
\hline 83925 & 90880 & G0084 & H0031, HM & H2023 & T1026 & 9467 \\
\hline 90801 & 90882 & G0085 & H0032 & H2024 & $\mathrm{T} 2010$ & 9468 \\
\hline 90802 & 90885 & G0086 & H0032, HK & H2025 & T2011 & 9469 \\
\hline 90804 & 90887 & G0087 & H0032, TS & H2026 & T2016 & 96101 \\
\hline 90805 & 90889 & G0088 & H0033 & $\mathrm{H} 2027$ & T2019 & 96102 \\
\hline 90806 & 90899 & G0089 & H0034 & H2028 & T2034 & 96118 \\
\hline 90807 & 95881 & G0090 & H0035 & H2029 & Z0001 & 96119 \\
\hline 90808 & 95882 & G0091 & H0036 & H2030 & Z0002 & 96103 \\
\hline 90809 & 95883 & G0092 & H0037 & H2031 & Z0002 & 96116 \\
\hline 90810 & 96100 & G0093 & H0038 & H2032 & Z0003 & 96120 \\
\hline 90811 & 96110 & G0094 & H0039 & $\mathrm{H} 2033$ & 9401 & \\
\hline 90812 & 96111 & G0176 & H0040 & H2034 & 9402 & \\
\hline 90813 & 96115 & G0176 & H0043 & H2035 & 9403 & \\
\hline 90814 & 96117 & G0177 & H0044 & H2036 & 9408 & \\
\hline 90815 & W1023 & G0442 & H0046 & H500 & 9409 & \\
\hline
\end{tabular}


Appendix 7.2 Chapter 4 supplemental table: Marginal effects estimated from multivariable difference-in-differences (DID) analyses of total healthcare costs and mental health (MH)-related costs among adults with mental health conditions (MHC), Optum Clinformatics ${ }^{\circledR}$ DataMart, 2010-2017

\begin{tabular}{|c|c|c|}
\hline & \multicolumn{2}{|c|}{ Marginal effect } \\
\hline & Total costs & MH costs \\
\hline & Mean $(95 \% \mathrm{CI})$ & Mean $(95 \% \mathrm{CI})$ \\
\hline TMH use (yes vs. no) & $-253.9(-1601.51093 .7)$ & $461.3(142.4,780.2)^{* *}$ \\
\hline Time (follow-up vs. baseline) & $-197.1(-307,-87.2)^{* * *}$ & $-269.9(-294.3,-245.5)^{* * *}$ \\
\hline DID & $554.3(-1005.2,2113.8)$ & $369.6(13.9,725.4)^{*}$ \\
\hline \multicolumn{3}{|c|}{ Predisposing factors ${ }^{a}$} \\
\hline Age (years) & $37.5(31.5,43.5)^{* * *}$ & $-11.0(-12.5-9.4)^{* * * *}$ \\
\hline Sex (female vs. male) & $-57.2(-212.0,97.6)$ & $-181.9(-219.4,-144.5)^{* * *}$ \\
\hline \multicolumn{3}{|c|}{ Enabling factors ${ }^{a}$} \\
\hline Medicare Advantage (yes vs. no) & $1257.3(995.3,1519.3)^{* * *}$ & $366.5(310.3,422.7)^{* * * *}$ \\
\hline HMO (yes vs. no) & $-449.9(-675.8-224.1)^{* * *}$ & $115.7(61.3,170.1)^{* * *}$ \\
\hline \multicolumn{3}{|c|}{ Need factors ${ }^{a}$} \\
\hline Any Severe MHC (yes vs. no) & $2199.8(2036.5,2363.1)^{* * * *}$ & $328.9(293.8,364.1)^{* * *}$ \\
\hline Any MH specialist visit (yes vs. no) & $156.2(0.6,311.9)^{*}$ & $201.5(167.9,235.1)^{* * *}$ \\
\hline \multicolumn{3}{|l|}{ Number of physical chronic conditions ${ }^{b}$} \\
\hline One & $2492.4(2279.5,2705.3)^{* * * *}$ & $362.7(309.7,415.6)^{* * *}$ \\
\hline Two or more & $5152.4(4921.4,5383.5)^{* * *}$ & $458(406.5,509.6)^{* * *}$ \\
\hline \multicolumn{3}{|l|}{ None } \\
\hline Polypharmacy c (yes vs. no) & $5447.5(5285.3,5609.8)^{* * *}$ & $321.6(280.4,362.9)^{* * *}$ \\
\hline Any tobacco/alcohol use disorder (yes vs. no) & $3981.2(3812.8,4149.6)^{* * *}$ & $201.3(170.5,232.2)^{* * * *}$ \\
\hline Any drug use disorder (yes vs. no) & $4798.9(4440.0,5157.8)^{* * *}$ & $382.9(336.5,429.3)^{* * *}$ \\
\hline Obesity (yes vs. no) & $2108(1900.4,2315.7)^{* * *}$ & $65.7(5.6,125.9)^{*}$ \\
\hline \multicolumn{3}{|c|}{ Environmental factors } \\
\hline \multicolumn{3}{|l|}{ Poverty $^{d}$} \\
\hline High (3rd \& 4th quartile) & $81.7(-76.9,240.2)$ & $73.0(34.0,112.0)^{* * *}$ \\
\hline \multicolumn{3}{|l|}{ Low (1st \& 2nd quartile) } \\
\hline \multicolumn{3}{|l|}{ Rurality d $^{\mathrm{d}}$} \\
\hline High (3rd \& 4th quartile) & $-165.4(-329.1,-1.6)^{*}$ & $6.1(-36.5,48.8)$ \\
\hline \multicolumn{3}{|l|}{ Low (1st \& 2nd quartile) } \\
\hline \multicolumn{3}{|l|}{ Mental health provider shortage d } \\
\hline High (3rd \& 4th quartile) & $122.5(-46.7,291.6)$ & $-21.0(-63.4,21.4)$ \\
\hline \multicolumn{3}{|l|}{ Low (1st \& 2nd quartile) } \\
\hline \multicolumn{3}{|l|}{ TMH capacity ${ }^{\mathrm{e}}$} \\
\hline High (3rd \& 4th quartile) & $156.9(-27.5,341.2)$ & $-28.3(-75.9,19.2)$ \\
\hline \multicolumn{3}{|l|}{ Low (1st \& 2nd quartile) } \\
\hline Year of TMH use & $-164.5(-198.7-130.4)^{* * * *}$ & $-8.0(-16.8,0.8)$ \\
\hline Lagging time, days & $-2.7(-3.4,-2.0)^{* * *}$ & $-2.1(-2.2,-1.9)^{* * *}$ \\
\hline
\end{tabular}




\footnotetext{
Note: ${ }^{\text {a }}$ all patient-level factors were measured in the six-month period before and after index date based on deidentified claims of Optum Clinformatics ${ }^{\circledR}$ DataMart. The first TMH use date was used as index date for users and a pseudo-index date was randomly selected from all mental health service dates among non-users.

${ }^{\mathrm{b}}$ chronic conditions examined included arthritis, asthma, coronary artery disease, cardiac arrhythmias, congestive heart failure, chronic kidney disease, chronic obstructive pulmonary disease, dementia and related disorders, diabetes, hypertension, hyperlipidemia, osteoporosis, stroke.

${ }^{c}$ polypharmacy was defined as having five or more medications from different therapeutic classes in the 90 days before index date.

d poverty, rurality, and mental health provider shortage were measured as average percentages of counties under qualified for each condition from 2010-2016 based on data from the 2016-2017 Area Health Resources File.

e TMH capacity was measured as average percentages of mental health facilities with telemedicine from 20102016 based on data from the 2010-2016 National Mental Health Services Survey.

${ }^{*}, \mathrm{p}<0.05 ;{ }^{* *}, \mathrm{p}<0.01 ;{ }^{* * *}, \mathrm{p}<0.001$.
} 